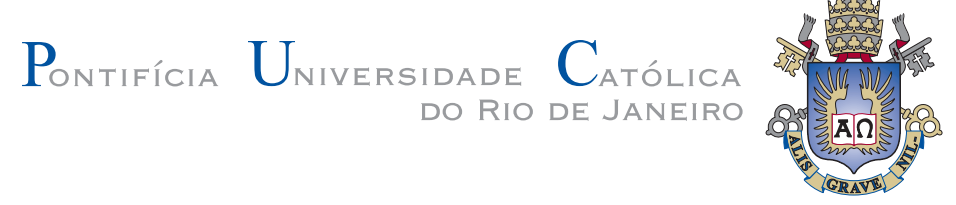

David Sebastian Calpa Juajinoy

\title{
Análise limite numérica de problemas axissimétricos em geotecnia
}

Dissertação apresentada como requisito parcial para obtenção do grau de Mestre pelo Programa de Pós-graduação em Engenharia Civil do Departamento de Engenharia Civil e Ambiental da PUCRio.

Orientadora : $\quad \operatorname{Prof}^{a}$. Raquel Quadros Velloso Coorientador: Prof. Eurípides do Amaral Vargas Junior Coorientador:

Dr. Fabricio Fernández 


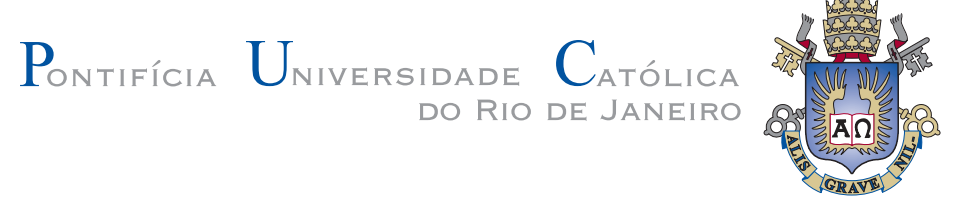

David Sebastian Calpa Juajinoy

\section{Análise limite numérica de problemas axissimétricos em geotecnia}

Dissertação apresentada como requisito parcial para obtenção do grau de Mestre pelo Programa de Pós-graduação em Engenharia Civil da PUC-Rio. Aprovada pela Comissão Examinadora abaixo.

Prof $^{a}$. Raquel Quadros Velloso

Orientadora

Departamento de Engenharia Civil e Ambiental - PUC-Rio

Prof. Eurípides do Amaral Vargas Junior Coorientador Departamento de Engenharia Civil e Ambiental - PUC-Rio

Dr. Fabricio Fernández

Coorientador

Departamento de Engenharia Civil e Ambiental - PUC-Rio

Prof. Luiz Fernando Campos Ramos Martha

Departamento de Engenharia Civil e Ambiental - PUC-Rio

Prof. Aldo Durand Farfán Departamento de Engenharia Civil - UENF 
Todos os direitos reservados. É proibida a reprodução total ou parcial do trabalho sem autorização da universidade, do autor e do orientador.

David Sebastian Calpa Juajinoy

Graduou-se em Engenharia Civil pela Universidade de Nariño (Pasto, Colômbia) em 2018. No ano 2019 ingressou no curso de Mestrado em Engenharia Civil da Pontificia Universidade Católica do Rio de Janeiro, na área de Geotecnia, onde vem desenvolvendo investigações na linha de pesquisa em Análise Limite Numérica.

Ficha Catalográfica

Calpa Juajinoy, David Sebastián

Análise limite numérica de problemas axissimétricos em geotecnia / David Sebastian Calpa Juajinoy; orientadora: Raquel Quadros Velloso ; co-orientadores: Eurípides do Amaral Vargas Junior, Fabricio Fernández. - 2021.

98 f. : il. color.; $30 \mathrm{~cm}$

Dissertação (mestrado) - Pontifícia Universidade Católica do Rio de Janeiro, Departamento de Engenharia Civil e Ambiental.

Inclui bibliografia.

1. Engenharia Civil e Ambiental - Tese. 2. Analise Limite Numerica;. 3. Programação cônica;. 4. Axissimetría;. 5. Poço;. 6. Mohr-Coulomb;. 7. Drucker-Prager.. I. Velloso, Raquel. II. Vargas, Eurípides. III. Fernández, Fabricio. IV. Pontifícia Universidade Católica do Rio de Janeiro. Departamento de Engenharia Civil e Ambiental. V. Título. 


\section{Agradecimentos}

Agradeço a Deus e à Virgem de Guadalupe pela oportunidade de realizar o sonho de estudar geotecnia num país tão maravilhoso como é o Brasil.

Á professora Raquel, pelo acompanhamento contínuo no desenvolvimento deste trabalho, ao professor Vargas pelas ideias e suggestões aportadas ao trabalho, ao doutor Fabricio Fernández pelos conhecimentos transmitidos, ao engenheiro Jhonatan Garcia pela ajuda e amizade.

Agradeçõ aos meus pais Marleny e Juan por serem sempre luz e amor na minha vida, aos meus irmãos, Juan, Mauricio, Andrea, Gabriela, e em especial ao José Luis, quem tem acreditado em mim sempre e fez muitos sacrificios para me ajudar a alcançar esta meta. À Juanita, Salomé e ao Nicolás pelos incontáveis momentos de alegría e diversão.

Aos meus amigos: Freddy, Fernando, Lizardo, Erland, Raul, John Harry, Cathe, Jennifer, Mauricio, Juan Pablo, Jhohana, Alessandra, Luiza, Ana, Thiago, Pilar e Marcelle.

À CAPES e à PUC-Rio, pelos auxílios concedidos, sem os quais este trabalho não poderia ser realizado. $\mathrm{O}$ presente trabalho foi realizado com apoio da Coordenação de Aperfeiçoamento de Pessoal de Nível Superior Brasil (CAPES) - Código de Financiamento 001.

A meus familiares, amigos e amigas por serem parte da minha vida. Finalmente, dedico este trabalho ao meu tio José, que recentemente faleceu e deixou uma saudade sem fim na minha familia. 


\section{Resumo}

Calpa Juajinoy, David Sebastián; Velloso, Raquel (Orientadora); Vargas, Eurípides (Co-orientador); Fernández, Fabricio (Coorientador). Análise limite numérica de problemas axissimétricos em geotecnia. Rio de Janeiro, 2021. 98p. Dissertação de Mestrado - Departamento de Engenharia Civil e Ambiental, Pontifícia Universidade Católica do Rio de Janeiro.

Este trabalho de dissertação de mestrado apresenta a implementação da análise limite numérica com formulação mista-fraca, baseada no teorema do límite inferior, e sua aplicação em problemas de estabilidade axissimétricos. A formulação com elementos finitos foi implementada no software Matlab, onde se estabelece o problema de otimização que compreende a definição da equação de equilibrio e a adaptação dos criterios de ruptura de Drucker-Prager e Mohr-Coulomb às programações cônica de segunda ordem e semidefinida, respectivamente, e que posteriormente é resolvido com o algoritmo Mosek Aps 9.2. Como resultado do problema de otimização o fator de colapso e o campo de velocidades podem ser obtidos, permitindo identificar o mecanismo de ruptura. $\mathrm{O}$ presente trabalho foca-se na análise de estabilidade de um poço que é executada em 3 fases, em função das condições consideradas no modelo. Os resultados obtidos da análise axissimétrica foram validados mediante analises em modelos tridimensionais e comparados com resultados dos softwares Plaxis 2D e Optum G2, também foram incluídos os resultados da modelagem MPM, com o sotware MPM-PUCRio. Por fim foi estudado o caso da capacidade de carga de uma fundação circular rasa, cujos resultados foram comparados com os apresentados por outros autores.

\section{Palavras-chave}

Analise Limite Numerica; Programação cônica; Axissimetría;

Poço; Mohr-Coulomb; Drucker-Prager. 


\section{Abstract}

Calpa Juajinoy, David Sebastián; Velloso, Raquel (Advisor); Vargas, Eurípides (Co-advisor); Fernández, Fabricio (Co-advisor). Numerical limit analysis of axisymmetric problems in geotechnical engineering. Rio de Janeiro, 2021. 98p. MSc. Dissertation Departamento de Engenharia Civil e Ambiental, Pontifícia Universidade Católica do Rio de Janeiro.

This work dissertation presents the implementation of numerical limit analysis with mixed-weak formulation, based on the the lower bound limit theorem and its application in axisymmetric stability problems. The finite element formulation was implemented in Matlab, where the optimization problem is established, which comprises the definition of the equilibrium equation and the adaptation of the Drucker-Prager and Mohr-Coulomb rupture criteria to the second-order cone programming and semidefined programming, respectively, and which is later solved with the Mosek Aps 9.2 algorithm. As a result of the optimization problem, the collapse factor and the speed field can be obtained, allowing to identify the rupture mechanism. The present work focuses on the stability analysis of a well that is carried out in 3 phases, depending on the conditions considered in the model. The results obtained in the axissymmetric analysis were validated through analysis in three-dimensional models and compared with results of plaxis 2D and Optum G2 software, also included the results of MPM modeling, with the software MPM-PUCRio. Finally, the case of the load capacity of a shallow circular foundation is studied, the results of which are compared with those presented by other authors.

\section{Keywords}

Numeric Limit Analysis; Conic Programming; Axissimetry; Well; Mohr-Coulomb; Drucker-Prager. 


\section{Sumário}

1 INTRODUÇÃO

$\begin{array}{lll}1.0 .1 & \text { Estado da arte } & 15\end{array}$

$\begin{array}{ll}1.0 .2 \text { Objetivos } & 16\end{array}$

$\begin{array}{ll}\text { 1.0.2.1 Objetivos específicos } & 16\end{array}$

$\begin{array}{ll}\text { 1.0.3 Estrutura do trabalho } & 17\end{array}$

2 ANÁLISE LIMITE $\quad 18$

2.1 Hipóteses da análise limite $\quad 19$

2.1.1 Considerações sobre o critério de escoamento 19

2.1.2 Comportamento tensão-deformação do material 21

2.1.3 Potencial plástico e Lei de fluxo associada 22

2.1.4 Postulado de estabilidade de Drucker 22

2.2 Critérios de escoamento 23

2.2.1 Critério de escoamento de Mohr-Coulomb 24

2.2.2 Critério de escoamento de Drucker-Prager 25

2.3 Principio dos trabalhos virtuais 28

2.4 Formulação da Análise Limite como problema de otimização 30

2.4.1 Problema primal 32

2.4.2 Problema dual 33

2.4.3 Algoritmos de otimização 34

3 IMPLEMENTAÇÃO NUMÉRICA $\quad 37$

$\begin{array}{lll}3.1 & \text { Estado axissimétrico } & 37\end{array}$

3.2 Discretização do meio contínuo 38

3.2.1 Discretização espacial 38

3.2.2 Transformação de coordenadas $\quad 41$

3.2.3 Integração numérica 42

3.3 Formulação da equação de equilíbrio (restrição de igualdade) do problema de otimização $\quad 43$

3.3.1 Matriz de compatibilidade cinemática 43

$\begin{array}{ll}\text { 3.3.2 Matriz de Equilíbrio } & 45\end{array}$

$\begin{array}{ll}\text { 3.3.3 Vetores de cargas nodais } & 45\end{array}$

3.3.4 Formulação matricial da equação de equilíbrio 46

3.4 Transformação dos critérios de escoamento ao espaço cônico 47

3.4.1 Critério de escoamento de Drucker-Prager na forma Cônica de Segunda Ordem, sem poropressões $\quad 47$

3.4.2 Critério de escoamento de Drucker-Prager na forma Cônica de Segunda Ordem, considerando poropressões 49

3.4.3 Critério de escoamento de Mohr-Coulomb na forma Semidefinida 50

3.5 Considerações finais da implementação numérica 52

3.5.1 Condições de contorno 52

3.5.2 Estimação do fator de segurança 52

3.5.3 Obtenção do mecanismo de colapso 53

3.5.4 Diagrama de fluxo geral da implementação 53 
4 RESULTADOS

4.1 Fase 1: Analise de estabilidade de poço 56

4.1.1 Considerações do caso estudado 56

4.1.2 Validação Analise Axissimétrico - Analise Tridimensional 56

$\begin{array}{ll}\text { 4.1.3 Analise de refinamento da malha } & 60\end{array}$

4.1.4 Analise de estabilidade para diferentes parâmetros do solo 61

4.1.5 Analise de estabilidade de poço considerando heterogeneidade 64

4.1.5.1 Caso 1: Camada mais resistente na base 64

4.1.5.2 Caso 2: Presença de camada menos resistente 66

4.1.6 Comparação com resultados presentes na literatura, em termos do número de estabilidade $\quad 67$

4.2 Fase 2: Analise de estabilidade de poço com anel de concreto 70

$\begin{array}{lll}4.2 .1 & \text { Condições do problema estudado } & 70\end{array}$

4.2.1.1 Modificação da equação de equilíbrio $\quad 71$

4.2.1.2 Calibração dos parâmetros M-C do anel de concreto 72

4.2.2 Validação Analise Axissimétrico - Analise Tridimensional 72

4.2.3 Analise de estabilidade para diferentes parâmetros M-C do solo 75

$\begin{array}{lll}\text { 4.2.4 Analise comparativo ALN-MPM } & 78\end{array}$

4.2.5 Analise de estabilidade do poço com anel de concreto, considerando heterogeneidade 80

4.3 Fase 3: Analise de estabilidade de poço com anel de concreto, considerando a presença do nível da água $\quad 82$

4.3.1 Analise comparativo ALN-MPM 84

4.4 Analise da capacidade de carga de uma fundação circular rasa 86

$\begin{array}{llr}5 & \text { Conclusões } & 90\end{array}$

$\begin{array}{lr}\text { Referências Bibliográficas } & 93\end{array}$ 


\section{Lista de figuras}

2.1 Superfície de escoamento no espaço das tensões principais. 20

2.2 Comportamento tensão-deformação de um material RPP.

2.3 Desenho esquemático da relação entre a superfície de escoamento e os vetores de incremento de tensão e vetor da taxa de deformação plástica.

2.4 Superfície de escoamento e vetor da taxa de deformação plástica, adaptado de [25].

2.5 Superfície de escoamento de Mohr-Coulomb (a) projetado no plano $\pi$ (b) no espaço das tensões principais.

2.6 Critérios de escoamento de Drucker-Prager e Von Mises no espaço das tensões principais.

2.7 Representação das diferentes configurações do cone de D-P e o critério de M-C no plano $\pi$.

2.8 Variação de $\alpha$ com o valor de $\phi$ para os diferentes tipos de cone D-P. Modificado de [14].

2.9 Representação gráfica dos conjuntos de termos da Equação do Princípio de Trabalhos Virtuais (a) Equilíbrio (b) Compatibilidade.

3.1 Tensões atuantes em um elemento diferencial na condição axissimétrica

3.2 Elemento finito quadrilateral isoparamétrico de 4 nós (Q4), em coordenadas locais

3.3 Representação gráfica da função de interpolação do nó 1 de um elemento bilinear tipo Q4

3.4 Mapeamento do elemento mestre (b) para o elemento real (a). $\quad 40$

3.5 Estimação do fator de segurança na ALN 53

3.6 Diagrama de fluxo geral da implementação da ALN 54

4.1 Geometria do poço estudado.

4.2 Geometria e malha semi-estruturada tridimensional do poço empregada na validação da análise axissimétrica.

4.3 Geometria e malha não estruturada plana do poço empregada na validação da análise axissimétrica.

4.4 Mecanismo de colapso obtido na análise tridimensional (caso 3). 59

4.5 Mecanismo de colapso obtido na análise axissimétrica (caso 3). 59

4.6 Analise de refinamento da malha.

4.7 Mecanismos de colapso obtidos na análise de refinamento geral da malha, com programação SDP M-C(a) 198 elementos (b) 1371 elementos (c) 5404 elementos.

4.8 Comparação entre os resultados obtidos com os cones D-P (SOCP) e M-C (SDP).

4.9 Comparação dos resultados conseguidos neste trabalho com os resultados obtidos em Optum G2 e Plaxis 2D.

4.10 Mecanismo de colapso para o caso 7. (a) Análise limite axissimétrica, programação SDP-MC (b) Optum G2 (c) Plaxis 2D. 
4.11 Geometria e malha não estruturada do caso 1 de heterogeneidade.

4.12 Mecanismo de colapso, caso 1 de heterogeneidade. (a) Análise limite programação SDP M-C (b) Optum G2 (c) Plaxis 2D.

4.13 Geometria e malha não estruturada do caso 2 de heterogeneidade.

4.14 Mecanismo de colapso, caso 2 de heterogeneidade. (a) Análise limite programação SDP M-C (b) Optum G2 (c) Plaxis 2D.

4.15 Geometrias e malhas não estruturadas dos poços (a) relação $H / R=1$, malha de 9270 elementos (b) relação $H / R=3$, malha de 9814 elementos.

68

4.16 Mecanismo de colapso estimado para $\phi=20^{\circ}$ e $H / R=1$ por: (a) O presente trabalho SDP M-C (b) Sun e Yang (2020).

4.17 Mecanismo de colapso estimado para $\phi=20^{\circ}$ e $H / R=3$ por: (a) O presente trabalho SDP M-C (b) Sun e Yang (2020).

4.18 Resultados da análise de calibração dos parâmetros M-C do concreto. 73

4.19 Geometria e malha semi-estruturada tridimensional do poço com anel de concreto, empregada na validação da análise axissimétrica.

4.20 Geometria e malha não estruturada plana do poço com anel de concreto, empregada na validação da análise axissimétrica.

4.21 Mecanismo de colapso obtido na análise tridimensional, análise do poço com anel de concreto, SOCP D-P Cone 2, representação do caso 1.

4.22 Mecanismo de colapso obtido na análise axissimétrica, análise do poço com anel de concreto, SOCP D-P Cone 2, representação do caso 1.

4.23 Comparação dos resultados conseguidos neste trabalho com os resultados obtidos em Optum G2 e Plaxis 2D.

4.24 Mecanismo de colapso, poço com muro, caso 8. (a) Análise limite programação SDP M-C (b) Optum G2 (c) Plaxis 2D.

4.25 Modelo MPM tridimensional do poço com o anel de concreto, formado por 93702 pontos materiais.

4.26 Deslocamentos verticais finais para diversos $\mathrm{FR}$, avaliados no ponto de controle no centro do poço.

4.27 Mecanismo de ruptura obtido com MPM. As Figuras mostram o campo de deslocamentos verticais em metros, $\mathrm{FS}=6.5$.

4.28 Geometria e malha não estruturada utilizada no modelo do poço com anel de concreto, considerando heterogeneidade.

4.29 Mecanismo de colapso obtido para o analise de heterogeneidade da Fase 2 (a) Análise limite com programação SDP M-C (b) Plaxis 2D. 82

4.30 Distribuição das poropressões $(\mathrm{kPa})$ adotada na análise.

4.31 Mecanismo de colapso, análise do poço com muro considerando o efeito da poropressão, caso 5. (a) Análise limite com programação SOCP-DP Cone 1 (b) Optum G2.

4.32 Deslocamentos verticais finais para diversos $\mathrm{FR}$, avaliados no ponto de controle no centro do poço.

4.33 Mecanismo de ruptura obtido com MPM. As Figuras mostram o campo de deslocamentos verticais em metros, $\mathrm{FS}=3.0$.

4.34 Geometria e malha não estruturada de 17685 elementos empregada na analise da capacidade de carga da sapata circular rasa. 
4.35 Superfícies de ruptura identificadas para (a) $\phi=15^{\circ}$ (b) $\phi=20^{\circ}$ (c) $\phi=25^{\circ}$ (d) $\phi=30^{\circ}$. 


\section{Lista de tabelas}

2.1 Correspondência entre os parâmetros dos critérios D-P e M-C 26

3.1 Coordenadas locais dos pontos de integração e seus respectivos fatores de ponderação

4.1 Condições de contorno no modelo tridimensional do poço 57

4.2 Condições de contorno no modelo axissimétrico do poço 57

4.3 Comparação entre os resultados obtidos nas análises axissimétrica e tridimensional (SOCP D-P cone 2)

4.4 Fator de Segurança obtido para diferentes casos de parâmetros do solo na análise de estabilidade

4.5 Resultados caso 1 de heterogeneidade, com camada mais resistente na base

4.6 Fator de segurança estimado no caso 2 de heterogeneidade, com presença de camada menos resistente

4.7 Comparação do número de estabilidade $\left(N_{s}\right)$ obtido para diferentes valores de ângulo de atrito $(\phi)$

4.8 Parâmetros de solo utilizados na análise de calibração

4.9 Condições de contorno no modelo tridimensional do poço com anel de concreto

4.10 Condições de contorno no modelo axissimétrico do poço com anel de concreto

4.11 Comparação do Fator de Segurança obtido na análise axissimétrica e tridimensional, estabilidade do poço com anel de concreto

4.12 Fator de segurança obtido no análise de estabilidade do poço com anel de concreto para diferentes casos de parâmetros do solo

4.13 Parâmetros de resistência dos materiais para a modelagem MPM

4.14 Parâmetros das camadas de solo e resultados do analise de estabilidade do poço

4.15 Fator de segurança obtido na análise de estabilidade do poço com muro, considerando poropressão, para diferentes casos de parâmetros do solo

4.16 Parâmetros dos materiais para a modelagem MPM

4.17 Condições de contorno no modelo axissimétrico da sapata circular rasa

4.18 Comparação dos valores do fator de capacidade de carga $N_{c}$ obtidos para uma fundação circular rasa 


\section{Lista de símbolos e abreviações}

$\begin{array}{ll}\phi & \text { Ângulo de atrito } \\ c & \text { Coesão } \\ \gamma & \text { Peso específico } \\ E & \text { Módulo de Young } \\ v & \text { Coeficiente de Poisson } \\ k & \text { Condutividade hidráulica } \\ \alpha \text { e } \kappa & \text { Parâmetros do critério Drucker-Prager } \\ \lambda & \text { Fator de colapso }\end{array}$

AL

Análise Limite

\section{ALN}

Análise Limite Numérica

D-P

FS

Critério de escoamento de Drucker-Prager

FR

Fator de Segurança

$\mathrm{M}-\mathrm{C}$

Fator de Redução da resistência

MEF

Critério de escoamento de Mohr-Coulomb

RPP

Método dos Elementos Finitos

PTV

Rígido Perfeitamente Plástico

PL

Principio dos Trabalhos Virtuais

PNL

Programação Linear

Programação Não Linear

SOCP

Programação cônica de segunda ordem

SDP

Programação semi-definida

PPV

Principio das Potências Virtuais

TLI

Teorema do Limite Inferior

TLS

Teorema do Limite Superior

KKT

Karush Kuhn Tucker 


\section{1 INTRODUÇÃO}

Um dos aspectos mais relevantes da teoria da plasticidade é a habilidade de prever facilmente valores aproximados de carga de colapso em uma grande variedade de aplicações [1]. A Análise Limite (AL) utiliza os teoremas da plasticidade para estudar problemas de estabilidade, dentro dos quais tem-se os geotécnicos, com aplicação em fundações, taludes até escavações subterrâneas.

A Análise Limite Numérica (ALN) incorpora o método dos elementos finitos na discretização do meio contínuo, para representar a formulação contínua da AL de maneira aproximada e permite estudar problemas de grande complexidade. Uma vez discretizado o meio, é estabelecido o problema de otimização, onde são feitas aproximações pelos limites inferior ou superior para obter a solução requerida.

Ao longo do tempo, as técnicas empregadas no processo de otimização têm avançado, passando pela programação linear (Pastor [2], Pastor e Tugerman [3], Anderheggen e Knopfel [4], Sloan [5], Sloan e Kleeman [6]), a programação não linear (Lyamin e Sloan [7], Krabbenhøft e Damkilde [8]) e mais recentemente a programação cônica (Krabbenhøft et al. [9], Makrodimopoulos e Martin [10], Mohapatra [11], Tang et al. [12]), sendo esta última a que tem demonstrado notável eficiência na resolução de problemas de grande escala.

Em comparação com outras metodologias de análise, como o equilíbrio limite, bem difundido na prática da geotecnia, a ALN tem certas vantagens:

- Não precisa fazer suposições da superfície de ruptura;

- Considera a relação tensão-deformação a partir do critério de escoamento e a lei de fluxo associada.

E em relação à análise elastoplástica, a ALN tem demostrado ser mais eficiente (tempo de processamento) na estimação de cargas de colapso, principalmente para análises com modelos tridimensionais, razão pela qual nos últimos tempos vem ganhando terreno no campo da geotecnia. 
A ALN é um tema de investigação, na área de geotecnia do programa de engenharia civil da PUC-RIO, na qual tem-se estudado problemas com modelos bidimensionais (para problemas no estado plano de deformação) e tridimensionais, com aplicação em problemas de capacidade de carga de fundações, estabilidade de taludes e estimação de cargas de colapso em túneis, porém não tem-se referência de trabalhos focados no estudo de problemas na condição axissimétrica, por esse motivo neste trabalho foi implementado um código para estudá-los.

O código foi testado na análise de estabilidade de um poço, efetuada em diferentes etapas, e na estimação da capacidade de carga de uma fundação circular rasa.

\subsubsection{Estado da arte}

Na literatura é possível encontrar uma boa quantidade de referências de análises feitas para problemas sob estado plano de deformação e tridimensional, mas não há muitos trabalhos aplicados a análises de problemas na condição axissimétrica.

No trabalho de Pastor e Tugerman [3] foi feita uma analise rigorosa pelo limite inferior, com aplicação em escavações circulares e compressão de corpos de forma cilíndrica. O critério Mohr-Coulomb foi linearizado e também foi utilizada a hipótese de Harr-von Karman no tratamento das tensões.

Kumar e Khatri [13] aplicaram a formulação pelo limite inferior ao estudo da capacidade de carga de fundações circulares, implementaram o critério de Mohr-Coulomb com programação linear, seguindo a hipótese de Harr-von Karman na estimação da tensão circunferencial $\left(\sigma_{\theta \theta}\right)$ como a tensão principal menor, formulação do critério Mohr-Coulomb, na condição axissimétrica, proposta por Pastor e Tugerman.

Chakraborty e Kumar [14] apresentaram uma análise pelo limite inferior aplicado à estimação dos fatores de capacidade de carga de uma fundação circular, utilizando o critério de escoamento de Drucker-Prager e programação não linear no processo de otimização.

Tang et al. [12] utilizaram a formulação pelo limite inferior no estudo de problemas axissimétricos, implementando o critério de Mohr-Coulomb com programação cônica de segunda ordem, o critério neste trabalho foi 
representado como uma tripla restrição, que surge da suposição feita do valor da tensão circunferencial $\left(\sigma_{\theta \theta}\right)$ como tensão principal.

Mohapatra e Kumar [15] aplicaram uma formulação do limite superior na determinação da capacidade de carga de uma fundação circular, para isto foi utilizado o critério Mohr-Coulomb com programação semidefinida. A aplicação do critério a esse tipo de programação é feita diretamente, a partir das tensões principais e sem precisar fazer suposições de $\sigma_{\theta \theta}$.

Sun e Yang [16] apresentaram uma formulação pelo limite inferior, no estado axissimétrico, implementado o critério Mohr-Coulomb com programação semidefinida. Com aplicação em escavações circulares e na estimação de capacidade de carga de fundações circulares.

A diferença entre os trabalhos de ALN no estado axissimétrico mencionados acima, com aproximações rigorosas pelos limites inferior ou superior, e este trabalho, é que neste trabalho se usou uma formulação mista-fraca, que permite obter soluções aproximadas. As analises incluíram os critérios de Drucker-Prager e Mohr-Coulomb com programações cônica de segunda ordem e semidefinida respectivamente. O problema principal estudado foi o de um poço, similar aos estudos feitos para escavações circulares, mas com uma geometria e condições que não foram apresentadas anteriormente, como por exemplo a inclusão do efeito das poropressões, de heterogeneidade e a presença de um elemento de concreto na análise.

\subsubsection{Objetivos}

O principal objetivo deste trabalho é avaliar a implementação e aplicação da análise limite usando as programações cônica de segunda ordem e semidefinida na resolução de problemas na condição axissimétrica.

\subsubsection{Objetivos específicos}

- Implementar com o método dos elementos finitos, uma formulação numérica que permita calcular a solução de problemas geotécnicos na condição axissimétrica.

- Acondicionar os critérios de ruptura de Drucker-Prager e MohrCoulomb no formato da programação cônica.

- Utilizar o software Mosek Aps 9.2 no processo de otimização.

- Validar a metodologia proposta para a condição axissimétrica mediante análises tridimensionais. 
- Verificar os resultados obtidos pelas metodologias implementadas neste trabalho com os obtidos nos softwares comerciais Plaxis 2D e Optum G2, em termos do fator de segurança.

- Avaliar as aproximações feitas com o critério Drucker-Prager ao critério Mohr-Coulomb.

- Definir o mecanismo de colapso mediante o campo de velocidades conseguido da solução do problema dual de otimização. E fazer a comparação com os mecanismos de colapso obtidos com Plaxis 2D e Optum G2.

- Incluir nas análises do poço o efeito da heterogeneidade, considerando a presença de mais de uma camada de solo.

- Definir parâmetros Mohr-Coulomb de resistência do concreto, que permitam considerar rígido, o comportamento desse material nas análises.

- Considerar o efeito das poropressões na análise de estabilidade.

- Comparar os resultados apresentados por outros autores no estudo do problema do poço e de fundações, em termos do número de estabilidade $N_{e}$ e o fator de capacidade de carga $N_{c}$, respectivamente.

\subsubsection{Estrutura do trabalho}

Este trabalho foi subdividido em 5 capítulos, que são descritos em seguida:

- Capítulo 1: Inclui uma breve introdução, o estado da arte, os objetivos da pesquisa e a estrutura do trabalho.

- Capítulo 2: Apresenta a base teórica da Analise Limite que engloba tanto os teoremas de plasticidade, as hipóteses da analise limite, fundamentos dos critérios de escoamento e a formulação da Analise Limite como um problema de otimização.

- Capítulo 3: Descreve inicialmente aspectos do estado axissimétrico, detalha a implementação numérica e o acondicionamento dos critérios de escoamento à programação cônica.

- Capítulo 4: Contém a descrição e os resultados dos exemplos de aplicação e a discussão dos mesmos.

- Capítulo 5: Resume as principais conclusões do trabalho e contém algumas sugestões para futuras pesquisas. 


\section{2}

\section{ANÁLISE LIMITE}

A AL se refere ao uso dos teoremas dos limites inferior (TLI) e superior (TLS) da plasticidade, que juntos determinam os valores limites da carga teórica de colapso plástico de uma determinada estrutura. Os teoremas da plasticidade foram apresentados inicialmente por Drucker [17] e Hill [18], com aplicação em metais, conceitos que foram posteriormente estendidos para o campo da geotecnia, também destacam os trabalhos de Chen [19] e Chen e Liu [20].

A predição das cargas de colapso está totalmente focada em encontrar as forças de volume e de superfície que levaram a estrutura ao estado de colapso iminente, sem se preocupar com o que acontece antes o depois, no sentido de tentar analisar deformações elásticas ou plásticas [1].

$\mathrm{Na}$ análise pelo TLI, se podem assumir infinitas configurações do estado de tensões no qual o corpo permanece em equilíbrio e atende tanto ao critério de ruptura, quanto as condições de contorno; se o campo de tensões dentro da massa do solo é estável e estaticamente admissível, o colapso não acontece, isto é, a carga aplicada é inferior à carga de colapso real teórica [21]. Portanto a maior carga estimada, sob essas considerações, será a mais próxima à carga de colapso real teórica.

$\mathrm{Na}$ análise pelo TLS, se podem assumir infinitas configurações do campo de velocidades no qual se satisfazem a condição de compatibilidade (velocidade-taxa de deformação), as condições de contorno de velocidades e a lei de fluxo associada (campos cinematicamente admissíveis). O campo de velocidades será determinado pela igualdade da taxa do trabalho externo com a taxa de dissipação de energia interna [17], obtendo para cada configuração possível do campo de velocidades uma carga de colapso que será maior do que a carga de colapso real teórica. Portanto a menor carga estimada, sob essas considerações, será a mais próxima à carga de colapso real teórica.

A partir do TLI e TLS, pode-se enunciar um teorema complementar, o 
teorema de unicidade, que garante a existência de uma solução única para a carga de colapso, de forma a satisfazer duas condições; a existência de um campo de tensões estaticamente admissível correspondente às tensões dentro ou na superfície de colapso, e a existência de um campo de deslocamentos geometricamente admissível. Quando ambas as condições são satisfeitas simultaneamente o fator de carga encontrado é igual ao fator de carga de colapso, que é assim determinado de maneira única [22].

\subsection{Hipóteses da análise limite}

Para aplicar os teoremas da AL é necessário estabelecer hipóteses referentes ao comportamento mecânico do material e definir alguns conceitos importantes da teoria da plasticidade que serão apresentadas em seguida, e que são estudados também nos trabalhos desenvolvidos na linha de pesquisa da ALN na área de geotecnia do departamento de engenharia civil da PUC-RIO (Camargo [23], Carrión [24], Chahua [25], Farfán [26], García [27], Tapia [28]).

\subsubsection{Considerações sobre o critério de escoamento}

O critério de escoamento é uma condição que define o limite da elasticidade e o começo da geração de deformações plásticas em um corpo, sob qualquer possível estado de tensões. Na região elástica, se produzem deformações elásticas $\left(\varepsilon_{i j}^{e}\right)$, recuperáveis uma vez é removido o estado de tensões aplicado, porém, uma vez a condição de escoamento é alcançada, serão produzidas no corpo deformações permanentes, conhecidas como deformações plásticas $\left(\varepsilon_{i j}^{p}\right)$.

Para o estado unidimensional de tensões, o critério de escoamento é definido por um valor limite $\left(\sigma_{y}\right)$ da tensão que graficamente é representado por um ponto, já para o caso multiaxial o escoamento acontecerá quando o estado de tensões atinge uma superfície, conhecida como superfície de escoamento (Figura 2.1), que delimita os estados de tensão considerados admissíveis.

A mesma generalização pode ser estendida para a tensão de ruptura $\left(\sigma_{f}\right)$, admitindo a existência de uma função ou criterio $\mathrm{F}\left(\sigma_{i j}\right)$ que indica a ruptura do material quando $\mathrm{F}\left(\sigma_{i j}\right)=0$. Esta função também é interpretada como um critério de ruptura.

Em geral, se assume que as condições para o escoamento plástico de- 


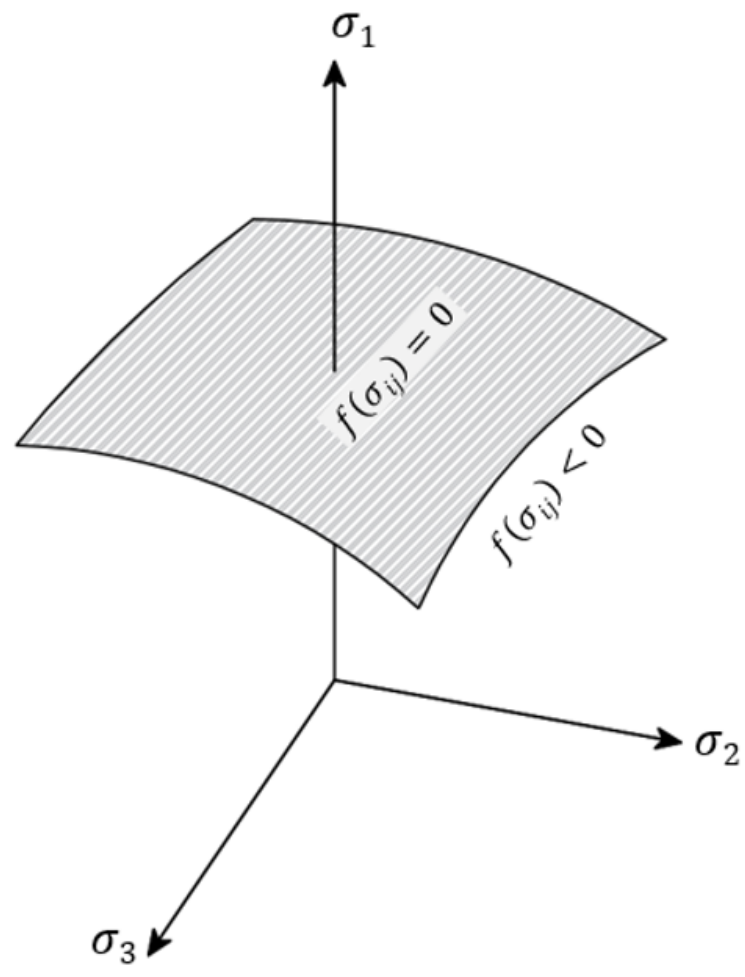

Figura 2.1: Superfície de escoamento no espaço das tensões principais.

pendem de uma função no campo tridimensional definido pelas tensões principais, que têm direções e valores determinados unicamente pela natureza do campo de tensão. Se o material possuir uma tendência natural a apresentar o escoamento independente da direção das tensões (material isotrópico), o critério de escoamento será função apenas das tensões principais [29]:

$$
f\left(\sigma_{i j}\right)=f\left(\sigma_{1}, \sigma_{2}, \sigma_{3}\right)=0
$$

A superfície de escoamento é convexa e de acordo com a posição do estado de tensões respeito a ela, o material vai apresentar um determinado comportamento [25], assim:

Estado de tensões $\left(\sigma_{i j}\right)$ no qual:

- $\mathrm{f}\left(\sigma_{i j}\right)<0 \Rightarrow$ Geração de deformações elásticas $\left(\varepsilon_{i j}^{e}\right)$

- $\mathrm{f}\left(\sigma_{i j}\right)=0 \Rightarrow$ Geração de deformações plásticas $\left(\varepsilon_{i j}^{p}\right)$

- $\mathrm{f}\left(\sigma_{i j}\right)>0 \Rightarrow$ Estado de tensões inadmissível 


\subsubsection{Comportamento tensão-deformação do material}

Na AL se considera o comportamento do material como rígido perfeitamente plástico (RPP), onde as deformações elásticas são desconsideradas $\left(\varepsilon_{i j}^{e}=0\right)$ e o material sofre deformações plásticas ilimitadas $\left(\varepsilon_{i j}^{p} \rightarrow \infty\right)$, uma vez é atingido o valor da tensão de escoamento $\sigma_{y}$ (Figura 2.2).

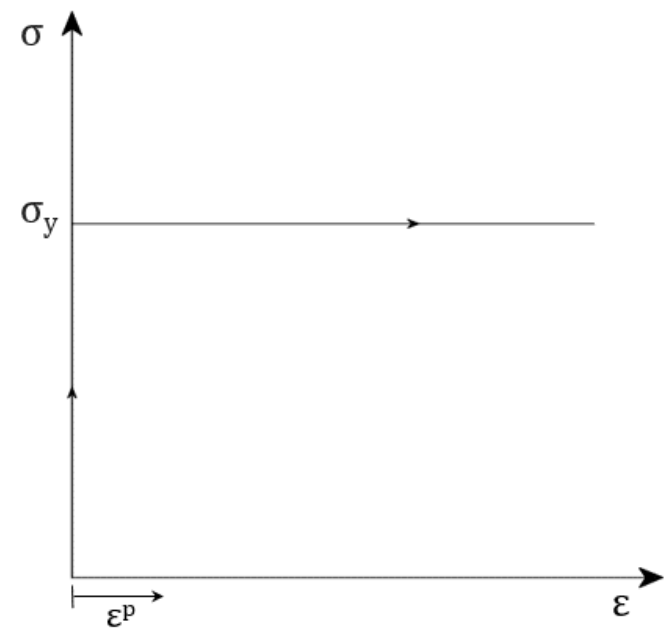

Figura 2.2: Comportamento tensão-deformação de um material RPP.

O material não apresenta nem endurecimento nem amolecimento, portanto a superfície de escoamento $f\left(\sigma_{i j}\right)$ fica fixa no espaço das tensões principais e coincide com a superfície definida pelo critério de ruptura $F\left(\sigma_{i j}\right)$. Sendo assim é possível escrever que determinado ponto do material RPP se deforma plasticamente quando:

$$
F\left(\sigma_{i j}\right)=0 \quad \Rightarrow \quad d F=\frac{\partial F}{\partial \sigma_{i j}} d \sigma_{i j}=0
$$

Na Equação anterior a direção do gradiente $\partial F / \partial \sigma_{i j}$ é perpendicular à superfície de ruptura num ponto de tensão $\left(\sigma_{i j}\right)$ e pela condição de consistência [30] o acréscimo de tensões $d \sigma_{i j}$ é tangente à superfície de ruptura, evitando assim ultrapassar o valor correspondente à tensão de ruptura.

Sob esta consideração do comportamento do material, a relação tensãodeformação não é bem estabelecida pois para o valor de $\sigma=\sigma_{y}$ a deformação plástica é indeterminada e para $\varepsilon=0$ o valor da tensão para $\sigma<\sigma_{y}$ não é único. Também não vai ser possível considerar o efeito do histórico de carregamentos. 


\subsubsection{Potencial plástico e Lei de fluxo associada}

Como foi dito na Secção 2.1.2, ao se considerar o solo como um material RPP uma das complicações que vai-se apresentar é estabelecer uma relação entre tensão e deformação (para estimar as deformações plásticas uma vez a superfície de escoamento é atingida), a AL supera esta dificuldade ao utilizar taxas de deformações plásticas $\left(\dot{\varepsilon}_{i j}^{p}\right)$ ao invés de deformações plásticas $\left(\varepsilon_{i j}^{p}\right)$, que podem ser obtidas a partir da seguinte expressão chamada de lei de fluxo $[18,32,33]$ :

$$
\dot{\varepsilon}_{i j}^{p}=\dot{\gamma} \frac{\partial Q\left(\sigma_{i j}\right)}{\partial \sigma_{i j}}
$$

onde $\dot{\gamma}$ é um escalar positivo, chamado de multiplicador plástico e

$$
Q=Q\left(\sigma_{i j}\right)=Q\left(\sigma_{1}, \sigma_{2}, \sigma_{3}\right)=0
$$

é conhecida como função potencial plástico, utilizada pela primeira vez por [32], de acordo com [18]. A formulação da Equação (2-3) foi baseada na observação feita por Saint-Venant em 1870, em metais, referente à coaxialidade entre os eixos principais das taxas de deformação plástica e os eixos das tensões principais . Se a função potencial plástico é a mesma superfície de escoamento $\left(Q\left(\sigma_{i j}\right)=f\left(\sigma_{i j}\right)\right)$, então a lei de fluxo é denominada lei de fluxo associada [36], a Equação (2-3) pode ser reescrita como:

$$
\dot{\varepsilon}_{i j}^{p}=\dot{\gamma} \frac{\partial f\left(\sigma_{i j}\right)}{\partial \sigma_{i j}}
$$

\subsubsection{Postulado de estabilidade de Drucker}

De acordo com Drucker um material é dito estável quando um acréscimo de tensões sempre resulta em trabalho positivo, partindo desde qualquer ponto dentro da superfície definida pelo critério de escoamento. $\mathrm{Ou}$ seja:

$$
\left(\sigma_{i j}-\sigma_{i j}^{0}\right) \dot{\varepsilon}_{i j}^{p} \geq 0
$$

onde $\sigma_{i j}^{0}$ é o estado de tensões inicial em um ciclo de carregamento, $\sigma_{i j}$ é o estado de tensões no qual o material atinge a superfície de escoamento e $\varepsilon_{i j}^{p}$ é a taxa de deformação plástica correspondente a esse estado de tensões.

Na Figura(2.3) para uma taxa de deformação plástica $\left(\dot{\varepsilon}_{i j}^{p}\right)$, seu estado 
de tensão correspondente $\left(\sigma_{i j}\right)$ representa um ponto $A$ no espaço de tensões, o estado de tensões inicial $\left(\sigma_{i j}^{0}\right)$ é representado pelo ponto $O$, resultando no vetor $\overrightarrow{O A}$ de acréscimo de tensão. O vetor $\overrightarrow{A B}$ representa o vetor de taxa de deformação plástica, sendo assim, a Equação (2-6) representa um produto escalar dos vetores $\overrightarrow{O A}$ e $\overrightarrow{A B}$.

Para que o material seja estável (atenda à Equação (2-6)) independentemente do estado de tensões é preciso que $\overrightarrow{A B}$ seja perpendicular ao critério de escoamento $\left(F\left(\sigma_{i j}\right)=0\right)$, e além disso é necessário que o ângulo formado pelos vetores $\overrightarrow{A B}$ e $\overrightarrow{O A}$ seja agudo, condição que somente poderá ser cumprida se o critério de escoamento é convexo.

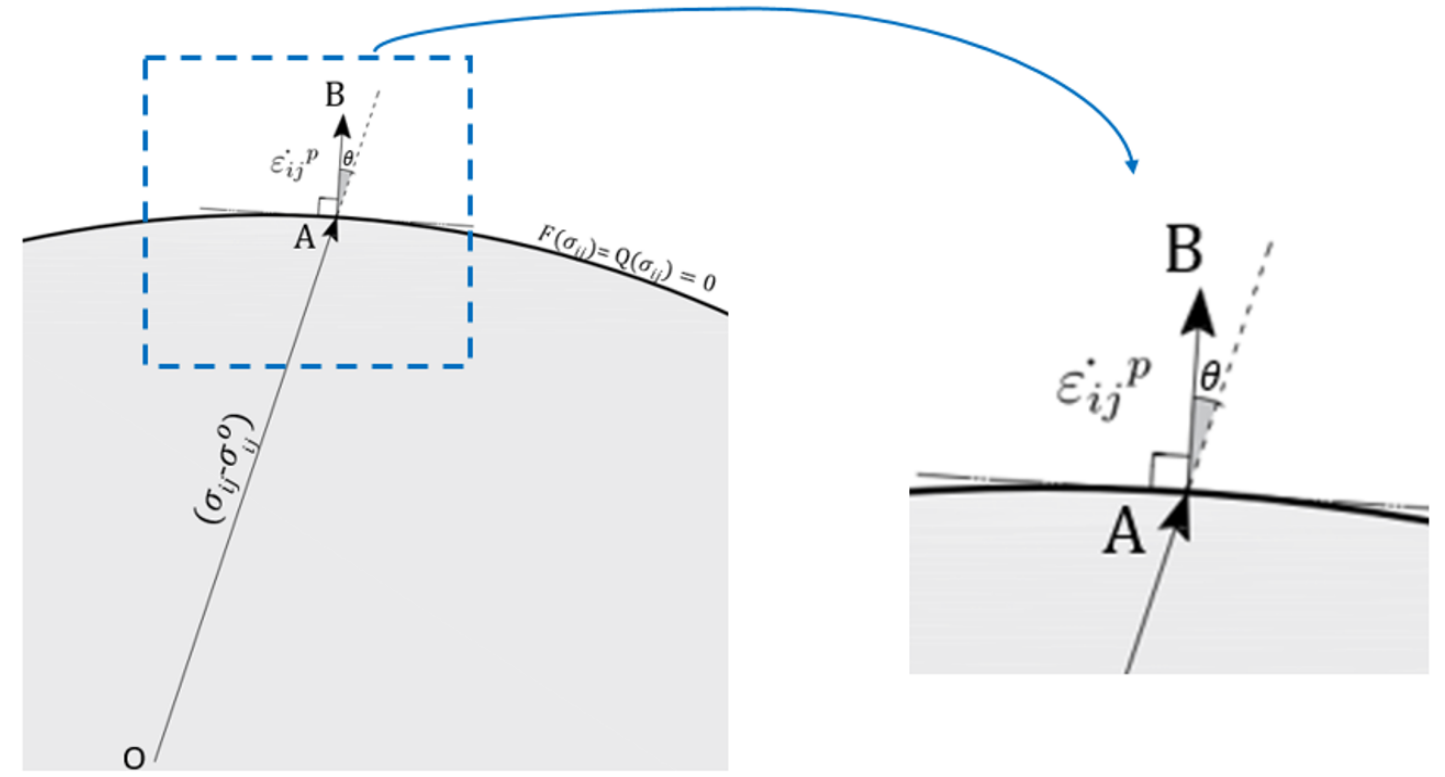

Figura 2.3: Desenho esquemático da relação entre a superfície de escoamento e os vetores de incremento de tensão e vetor da taxa de deformação plástica.

No espaço das tensões principais a relação entre a superfície de escoamento e a taxa de deformação plástica para materiais estáveis pode ser representada pela Figura 2.4.

\subsection{Critérios de escoamento}

Como foi revisto na Secção (2.1), no âmbito da AL os critérios de ruptura são empregados como critérios de escoamento. Neste trabalho foram 


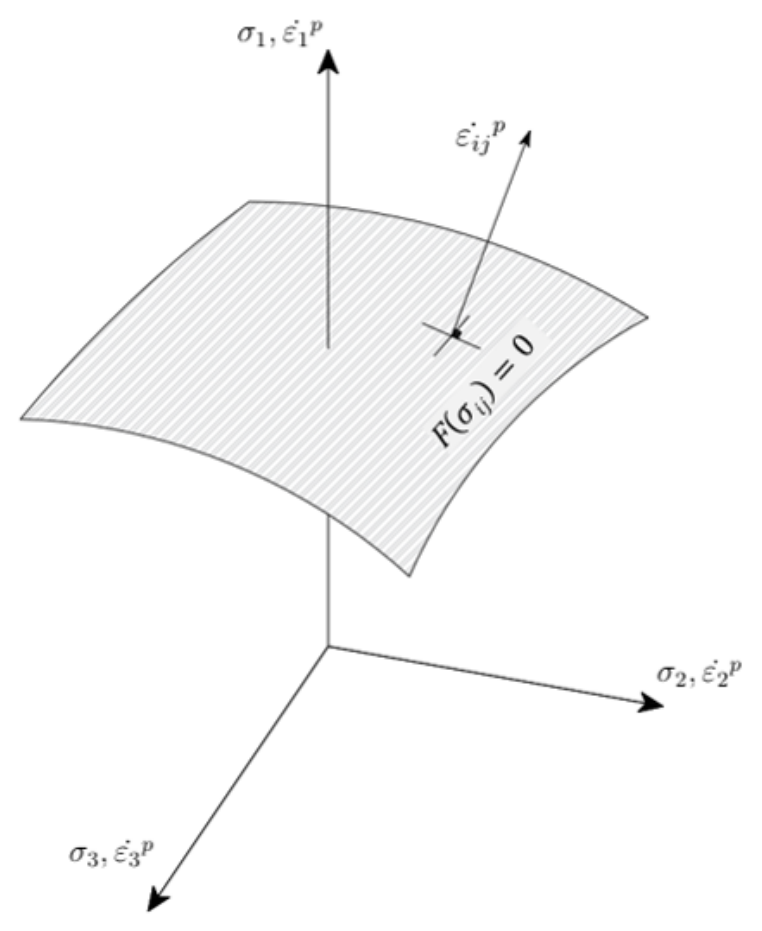

Figura 2.4: Superfície de escoamento e vetor da taxa de deformação plástica, adaptado de [25].

utilizados os critérios de Mohr-Coulomb e de Drucker-Prager (1952), no estado tridimensional e axissimétrico.

\subsubsection{Critério de escoamento de Mohr-Coulomb}

O critério de Mohr-Coulomb (M-C) pode ser considerado como uma combinação de duas hipóteses propostas por Coulomb (1736 - 1806) e Mohr (1835 - 1918). Coulomb admitiu uma envoltória de ruptura linear na determinação de uma combinação crítica de tensões normais e cisalhantes que causa ruptura em algum plano, enquanto que Mohr estabeleceu o critério de ruptura, linear ou não, escrito em termos das tensões principais maior e menor atuantes no plano de ruptura, no momento da ruptura. Essas duas hipóteses foram combinadas resultando no critério de resistência ao cisalhamento de solos padrão para os engenheiros geotécnicos. A função de escoamento em termos das tensões principais $\left(\sigma_{1} \geq \sigma_{2} \geq \sigma_{3}\right)$, é dada pela seguinte Equação:

$$
f\left(\sigma_{i j}\right)=\sigma_{1}(1+\operatorname{sen} \phi)-\sigma_{3}(1-\operatorname{sen} \phi)-2 c \cos \phi \leq 0
$$

No espaço das tensões principais, o critério M-C define uma pirâmide 
de base hexagonal irregular e eixo coincidente com a diagonal principal (Figura 2.5). Neste trabalho se adotou uma convenção de sinais, para tensões de tração positivas e compressão negativas.

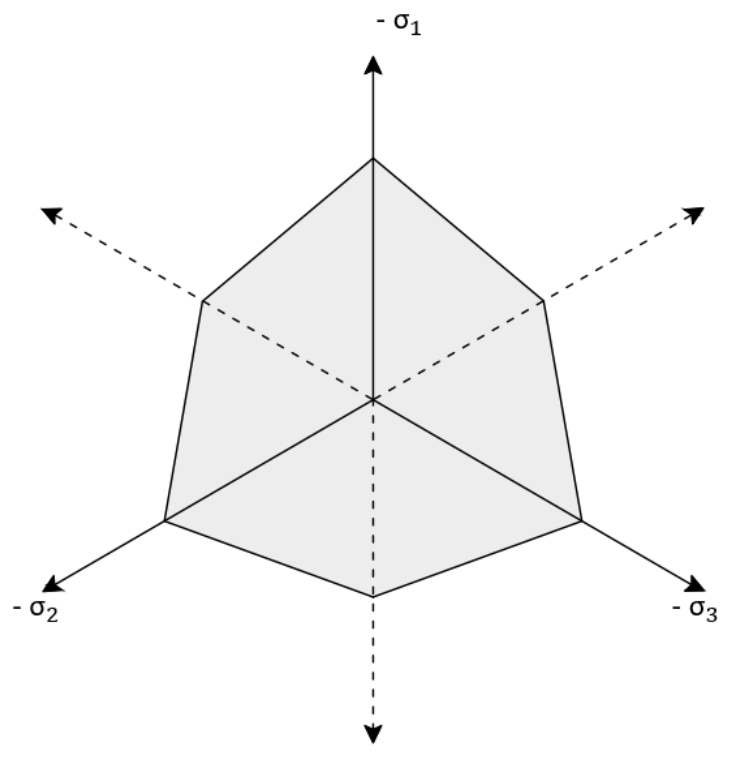

(a)

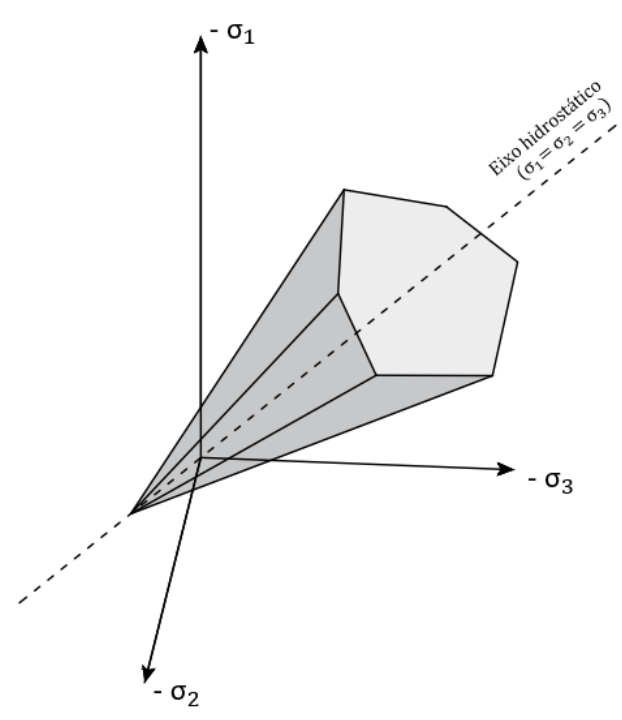

(b)

Figura 2.5: Superfície de escoamento de Mohr-Coulomb (a) projetado no plano $\pi$ (b) no espaço das tensões principais.

\subsubsection{Critério de escoamento de Drucker-Prager}

No espaço das tensões principais o critério Drucker-Prager (D-P) tem um formato de um cone, resultando em uma aproximação ao critério $\mathrm{M}-\mathrm{C}$ por uma única superfície, evitando os problemas no modelagem computacional gerados por as singularidades dos cantos. É considerado como uma generalização do critério de von Mises em que se introduz à expressão do critério de von Mises uma sensibilidade ao escoamento do material quanto à pressão hidrostática (Figura 2.6), obtendo-se a seguinte Equação:

$$
f\left(\sigma_{i j}\right)=\alpha I_{1}^{\prime}+\sqrt{J_{2}}-k \leq 0
$$

onde $I_{1}^{\prime}$ e $J_{2}$ são o primeiro invariante do tensor de tensões (efetivas) genérico e o segundo invariante do tensor de tensões de desvio respectivamente. Os parâmetros $\alpha$ e $\kappa$ podem ser determinados por regressão linear de resultados de ensaios triaxiais plotados no plano $\sqrt{J_{2}}$ versus $I_{1}^{\prime}$, ou obtidos em função dos parâmetros de resistência do critério M-C (ângulo de atrito 
$\phi^{\prime}$ e coesão $c^{\prime}$ ), fazendo coincidir no plano octaédrico o círculo do critério D-P com o hexágono irregular do critério M-C.

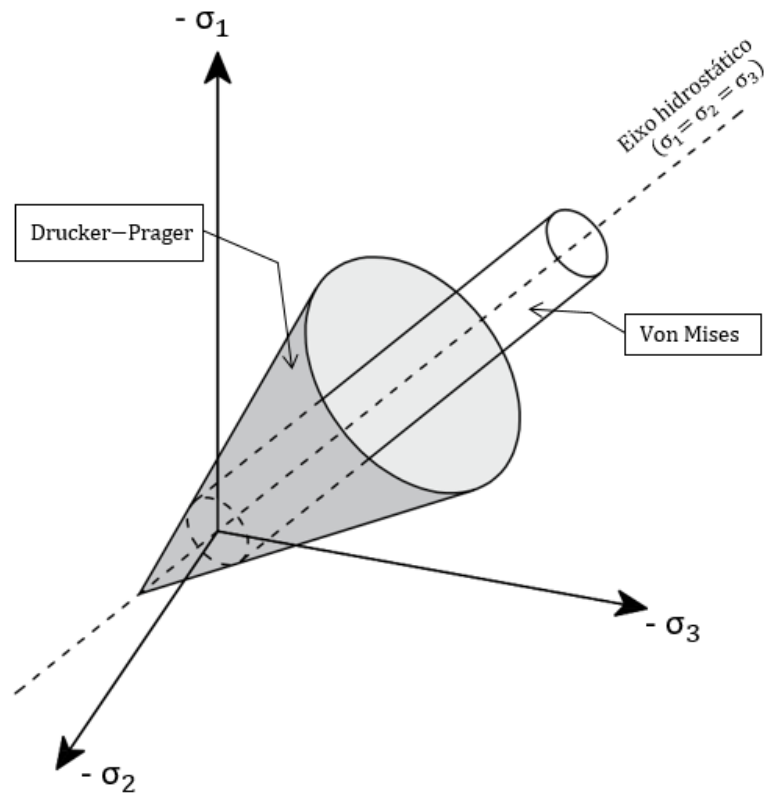

Figura 2.6: Critérios de escoamento de Drucker-Prager e Von Mises no espaço das tensões principais.

Em [14] são apresentadas as expressões para calcular os valores de $\alpha$ e $\kappa$ para varias configurações do cone D-P, indicadas na Tabela $2.1 \mathrm{e}$ representadas graficamente na Figura 2.7.

Tabela 2.1: Correspondência entre os parâmetros dos critérios D-P e M-C

\begin{tabular}{lcc}
\hline & \multicolumn{2}{c}{ Parâmetros do critério Drucker-Prager } \\
\cline { 2 - 3 } Tipo de cone & $\alpha$ & $\kappa$ \\
\hline Cone 1 (Inscrito) & $\frac{\sin \phi}{\sqrt{3}\left(3+\sin ^{2} \phi\right)^{1 / 2}}$ & $\frac{\sqrt{3} c \cos \phi}{\left(3+\sin ^{2} \phi\right)^{1 / 2}}$ \\
Cone 2 (Extensão triaxial) & $\frac{2 \sin \phi}{\sqrt{3}(3+\sin \phi)}$ & $\frac{2 \sqrt{3} c \cos \phi}{3+\sin \phi}$ \\
Cone 3 (Proposto C-K & $\frac{2 \sin \phi}{\sqrt{\xi}}$ & $\frac{6 c \cos \phi}{\sqrt{\xi}}$ \\
Cone 4 (Médio) & $\frac{2 \sin \phi}{3 \sqrt{3}}$ & $\frac{6 c \cos \phi}{3 \sqrt{3}}$ \\
Cone 5 (Compressão triaxial) & $\frac{2 \sin \phi}{\sqrt{3}(3-\sin \phi)}$ & $\frac{2 \sqrt{3} c \cos \phi}{3-\sin \phi}$ \\
\hline
\end{tabular}

$\left(^{*}\right)$ O valor de $\xi$ varia de 29.5 a 28 para $\phi=5^{\circ}$ e $\phi=45^{\circ}$ respectivamente. 


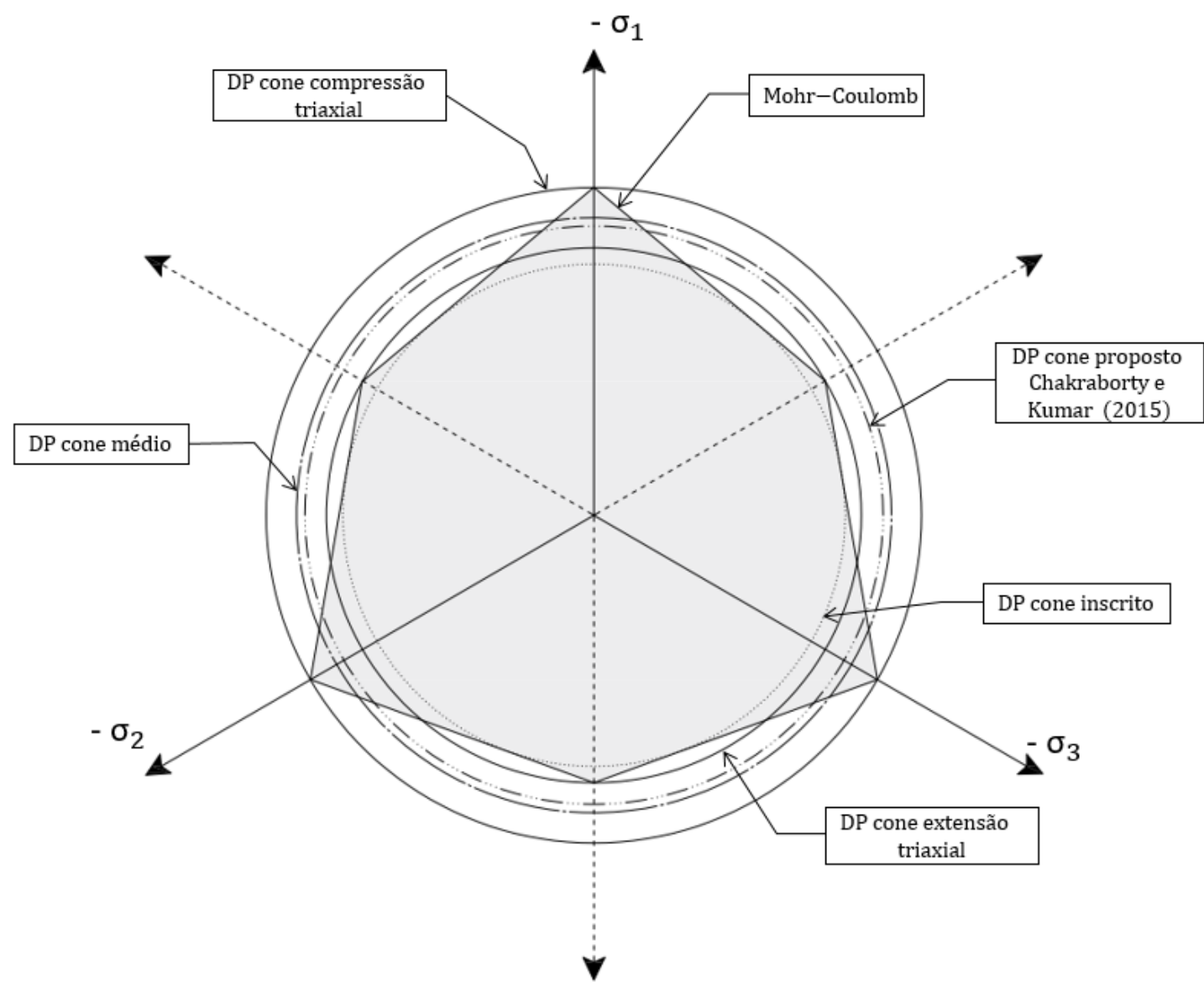

Figura 2.7: Representação das diferentes configurações do cone de D-P e o critério de M-C no plano $\pi$.

Na aproximação feita desde o critério D-P para o critério M-C, quando o círculo resultante da projeção do cone no plano $\pi$ coincide com as arestas de compressão do critério $\mathrm{M}-\mathrm{C}$, tem-se o cone de compressão triaxial, similarmente para o cone de extensão triaxial. O cone médio é baseado nos parâmetros definidos por Nayak e Zienkiewicz (1972) [34] e resulta em uma aproximação média dos cones de extensão e compressão [35]. O cone inscrito fica ajustado no interior do hexágono irregular M-C. Além do mais, exite uma adaptação do cone proposta no trabalho de Chakraborty e Kumar [14], próxima ao cone médio, porém inferior.

Alternativamente a Figura 2.8 apresenta a variação de $\alpha$ com o ângulo de atrito, para cada tipo de cone estudado. Estimando diretamente o valor de $\alpha$, o valor de $\kappa$ pode-se calcular a partir da seguinte Equação [14]:

$$
k=3 \alpha \cot \phi
$$




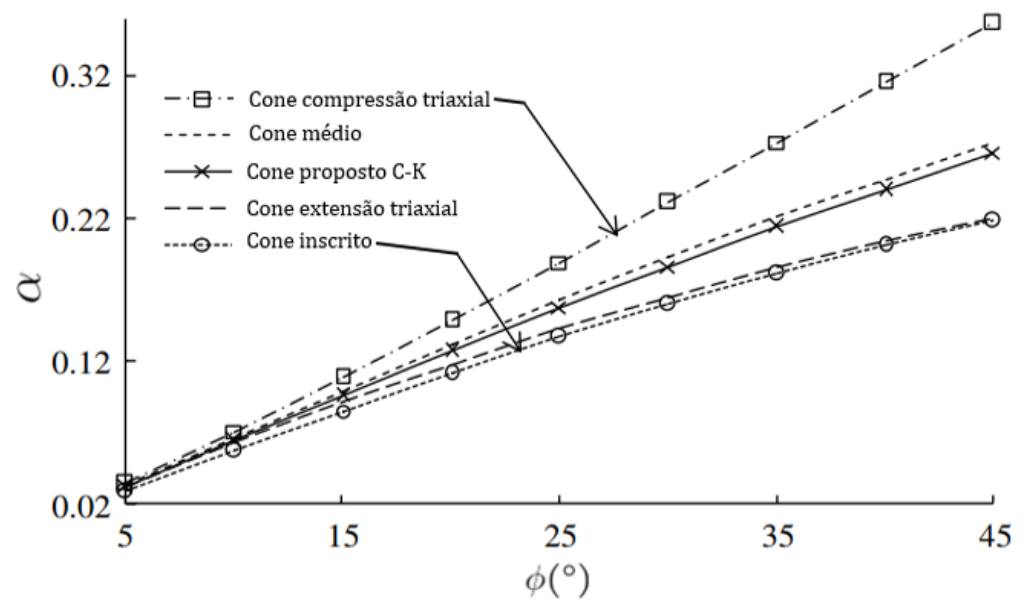

Figura 2.8: Variação de $\alpha$ com o valor de $\phi$ para os diferentes tipos de cone D-P. Modificado de [14].

\subsection{Principio dos trabalhos virtuais}

O principio dos trabalhos virtuais (PTV) é uma forma alternativa de expressar as condições de compatibilidade e equilíbrio [36]. Este principio relaciona um campo estaticamente admissível (ou em equilibro), com um campo cinematicamente admissível (ou compatível) [19].

Uma das hipóteses mais importantes que se fazem para aplicar o PTV, é que no momento do colapso, o corpo não sofre mudanças consideráveis na sua geometria, já que para todos os cálculos será empregada a geometria original. A Equação do trabalho virtual que trata com os campos de equilíbrio e compatibilidade, como conjuntos independentes, é apresentada em seguida:

$$
\begin{array}{r}
\int_{A} u_{i} T_{i} d A+\int_{V} u_{i} F_{i} d V=\int_{V} \sigma_{i j} \varepsilon_{i j} d V \\
-d W_{e x t}=\int_{A} u_{i} T_{i} d A+\int_{V} u_{i} F_{i} d V \rightarrow \text { Trabalho externo } \\
-d W_{\text {int }}=\int_{V} \sigma_{i j} \varepsilon_{i j} d V \quad \rightarrow \text { Trabalho interno }
\end{array}
$$

Na Equação (2-10) se pode identificar os dois conjuntos de termos referidos anteriormente, onde: $u_{i}$ (deslocamento virtual) e $\varepsilon_{i j}$ (deformação compatível com o deslocamento virtual), são parte do conjunto de compatibilidade e $T_{i}$ (forças de superfície), $F_{i}$ (forças de campo) e $\sigma_{i j}$ (Tensões em equilíbrio com as forças $T_{i}$ e $F_{i}$ ) são parte do conjunto de equilíbrio. 
Na Figura (2.9)(a), para que haja equilíbrio se devem cumprir as seguintes condições:

$-T_{i}=\sigma_{i j} n_{j} \quad \rightarrow$ Na superfície

$-\frac{\partial \sigma_{i j}}{\partial x_{j}}+F_{i}=0 \rightarrow$ No interior do corpo

- $\sigma_{i j}=\sigma_{j i} \quad \rightarrow$ Simetria do tensor de tensões

Em relação á Figura (2.9)(b), para garantir uma distorção de corpo compatível com o campo de deslocamentos virtuais, tem-se que respeitar a seguinte condição:

$$
-\varepsilon_{i j}=\frac{1}{2}\left(\frac{\partial u_{i}}{\partial x_{j}}+\frac{\partial u_{j}}{\partial x_{i}}\right)
$$

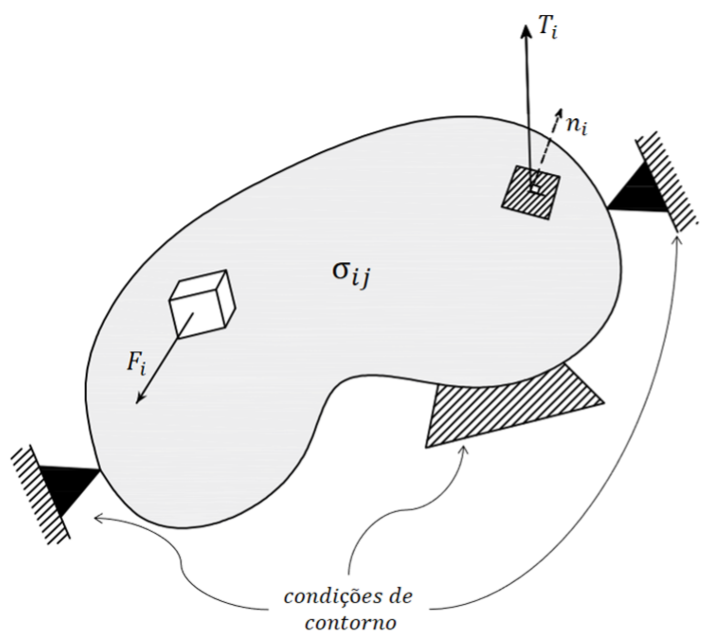

(a)

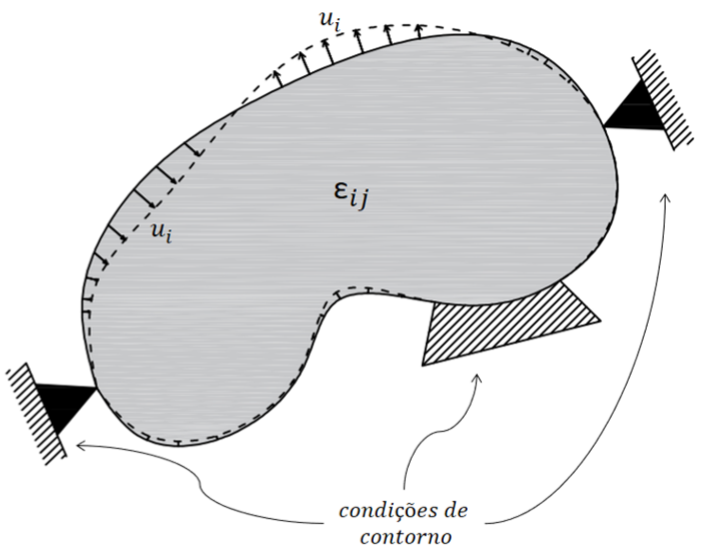

(b)

Figura 2.9: Representação gráfica dos conjuntos de termos da Equação do Princípio de Trabalhos Virtuais (a) Equilíbrio (b) Compatibilidade.

Dividindo a Equação(2-10) por $d t$ (variação infinitesimal de tempo) e assumindo um comportamento do material RPP $\left(\varepsilon_{i j} \Rightarrow \varepsilon_{i j}^{p}\right)$, tem-se:

$$
\int_{A} \dot{u}_{i} T_{i} d A+\int_{V} \dot{u}_{i} F_{i} d V=\int_{V} \sigma_{i j} \dot{\varepsilon}_{i j}^{p} d V
$$

- $\dot{W}_{e}=\int_{A} \dot{u}_{i} T_{i} d A+\int_{V} \dot{u}_{i} F_{i} d V \rightarrow$ Potência virtual pelas forças externas

$$
\text { - } \dot{P}=\int_{V} \sigma_{i j} \dot{\varepsilon}_{i j}^{p} d V \quad \rightarrow \text { Taxa de dissipação da energia interna }
$$


A Equação (2-11) é denominada Principio das Potências Virtuais (PPV), onde $\dot{u}_{i}$ é a velocidade virtual e $\dot{i}_{i j}^{p}$ é a taxa de deformação plástica.

Com base na Equação (2-11) para que o sistema esteja em equilíbrio, a potência virtual pelas forças externas $\left(\dot{W}_{e}\right)$ tem que ser menor ou igual à taxa de dissipação da energia interna do material $(\dot{P})$.

$$
\dot{W}_{e} \leq \dot{P}
$$

\subsection{Formulação da Análise Limite como problema de otimização}

Levando em conta as hipóteses de escoamento e comportamento do material na $\mathrm{AL}$, estudadas nas seções anteriores, para as quais são provados os teoremas da plasticidade rigorosamente $[1,19,20]$, podem ser formulados os teoremas da AL como problemas de otimização, onde o objetivo é estimar a carga de colapso da estrutura.

Na análise pelo limite inferior (formulação estática), procura-se maximizar a intensidade de uma carga predefinida para a qual o campo de tensões obtido é estaticamente admissível, atendendo às equações de equilíbrio e o critério de escoamento, em todo o domínio e as condições de contorno de tensão, no contorno do problema.

$\mathrm{Na}$ análise pelo limite superior (formulação cinemática), procura-se minimizar a intensidade de uma carga predefinida para a qual o campo de velocidades obtido seja cinematicamente compatível com o campo das taxas de deformação e atendam a lei de fluxo, em todo o domínio e as condições de contorno de velocidade, no contorno do problema.

Para a solução do sistema de equações de igualdade e desigualdade que surgem na análise, o meio contínuo é discretizado e é estimada uma solução aproximada utilizando o método dos elementos finitos. A solução obtida é uma estimação rigorosa do limite inferior ou do limite superior, dependendo do espaço de interpolação empregado para discretizar as tensões e velocidades.

Na formulação estática, tem-se que garantir o equilíbrio em todo o domínio apesar de ser aferido apenas em alguns pontos [23], por exemplo, é comum empregar elementos com variação linear das tensões, com presença de descontinuidades estaticamente admissíveis. Em comparação com elementos de ordem superior, utilizar o elemento linear tem a vantagem de somente 
precisar impor a restrição do critério de ruptura nos nós [10]. Nos trabalhos de Lysmer [37], Pastor [2] e Sloan [38] foram usados elementos triangulares de 3 nós (problemas 2D), com variação linear de tensões e descontinuidades de tensão compartilhadas por elementos adjacentes, obtendo estimativas rigorosas do limite inferior da carga de colapso. A diferença da formulação usualmente empregada no método dos elementos finitos, nesta formulação cada nó é particular e único para cada elemento, mais de um nó pode ter a mesma coordenada [12].

Na a formulação cinemática tem-se que garantir que a a lei de fluxo seja respeitada em todo o domínio, mesmo que ela somente pode ser imposta em alguns pontos [23]. Nos trabalhos de Bottero et al [39], Abdi et al [40], Sloan et al [6] e Lyamin e Sloan [7] foram selecionados elementos triangulares de 3 nós (problemas 2D), com variação linear do campo de velocidades no interior de cada elemento e com presença de descontinuidades adjacentes entre elementos. Makrodimopoulos e Martin [41] demostraram que também podem ser empregados elementos triangulares de 6 nós (problemas 2D), com uma distribuição quadrática do campo de velocidades, e com presença de descontinuidades, em que as taxas de deformação de cada elemento variam como uma combinação linear do valor das taxas de deformação dos vértices do elemento, obtendo uma estimação rigorosa do limite superior, melhor da obtida com o campo de velocidades linear, e com um custo computacional similar.

O objetivo principal do conhecimento dos limites rigorosos (inferior e superior) da carga de colapso, é a possibilidade de definir um intervalo de valores dentro do qual encontra-se a carga de colapso real teórica. Em Krabbenhøft et al [9] pode-se conferir que a medida que aumenta o refinamento da malha o intervalo estimado (e portanto o erro de estimação da carga de colapso real teórica) diminui.

Se o objetivo da análise não é estabelecer os valores limite da carga de colapso mas sim uma solução aproximada, podem-se empregar formulações mistas, a partir das quais podem ser obtidas soluções que não garantem o cumprimento a rigor de nenhum dos dois teoremas da AL. Nos trabalhos de Casciaro e Cascini [42], Christiansen [43], Christiansen e Andersen [44] e Zouain et al [45] é empregada essa técnica que permite obter um fator de colapso que necessariamente será inferior ao limite superior e superior ao limite inferior, sendo dessa maneira mais próximo ao fator de colapso real [23]. Em Aráujo [46] se demostrou que a formulação mista apresenta 
como vantagens principais a maior facilidade de implementação e o fato de possuir consistência em relação aos campos de tensões e velocidades obtidos, na busca de uma solução com aproximações nos campos estático e cinemático.

No presente trabalho (ao igual que em [23], [25], [47], [27]) é usada uma formulação mista-fraca, que tem um formato similar à formulação estática, porém satisfazendo aproximadamente as condições de equilíbrio e de contorno em tensão, e exatamente as condições de escoamento do material. É considerada fraca devido ao uso do principio dos trabalhos virtuais para a formulação das equações de equilíbrio.

\subsubsection{Problema primal}

Considerando uma formulação mista-fraca, o problema da AL é colocado como problema de otimização:

$$
\begin{array}{ll}
\text { maximizar } & \lambda \\
\text { sujeito a: } & \lambda\left(\int_{A} \dot{u}_{i} T_{i} d A+\int_{V} \dot{u}_{i} F_{i} d V\right)+\int_{A} \dot{u}_{i} T_{0 i} d A+\int_{V} \dot{u}_{i} F_{0 i} d V=\int_{V} \sigma_{i j} \dot{\varepsilon}_{i j}^{p} d V \\
& f\left(\sigma_{i j}\right) \leq 0
\end{array}
$$

onde $\lambda$ é o fator de colapso, a função objetivo do processo de otimização, um fator multiplicador que vai majorar as cargas responsáveis do colapso $\left(F_{i}, T_{i}\right)$, adicionalmente na Equação (2-13) tem-se cargas de volume $\left(F_{0 i}\right)$ e de superfície $\left(T_{0 i}\right)$ que ficam fixas, dependendo das condições do problema estudado. Tendo como variáveis do problema ao fator de colapso e as tensões, e duas restrições; uma de igualdade (referente ao equilíbrio) e uma de desigualdade (referente ao critério de escoamento).

Colocada na forma padrão do problema primal ou problema original de otimização, a Equação (2-13) pode ser rescrita como:

$$
\begin{array}{ll}
\text { minimizar } & (-\lambda) \\
\text { sujeito a: } & \lambda\left(\int_{A} \dot{u}_{i} T_{i} d A+\int_{V} \dot{u}_{i} F_{i} d V\right)+\int_{A} \dot{u}_{i} T_{0 i} d A+\int_{V} \dot{u}_{i} F_{0 i} d V-\int_{V} \sigma_{i j} \dot{\varepsilon}_{i j}^{p} d V=0 \\
& f\left(\sigma_{i j}\right) \leq 0
\end{array}
$$




\subsubsection{Problema dual}

É bem conhecida a dualidade entre os teoremas da AL, eles são construídos com base nos mesmos dados, mas considerando diferentes variáveis, suas soluções em termos da função objetivo são as mesmas [48]. Seguindo o mesmo procedimento efetuado em [27], para definir o problema dual obtido a partir do primal, é necessário definir a função Lagrangeana $\mathcal{L}$ :

$$
\begin{gathered}
\mathcal{L}\left(\sigma_{i j}, \mu, \rho\right)=-\lambda+\mu\left[\lambda\left(\int_{A} \dot{u}_{i} T_{i} d A+\int_{V} \dot{u}_{i} F_{i} d V\right)\right. \\
\left.+\int_{A} \dot{u}_{i} T_{0 i} d A+\int_{V} \dot{u}_{i} F_{0 i} d V-\int_{V} \sigma_{i j} \dot{\varepsilon}_{i j}^{p} d V\right]+\rho f\left(\sigma_{i j}\right)
\end{gathered}
$$

onde $\mu$ e $\rho$ são multiplicadores de Lagrange. A maximização da função $\mathcal{L}$ requer que no ponto crítico o gradiente seja nulo, então derivando a Equação (2-15) com respeito a $\sigma_{i j}$ tem-se:

$$
\frac{\partial \mathcal{L}}{\partial \sigma_{i j}}=-\mu \frac{\partial}{\partial \sigma_{i j}}\left(\int_{V} \sigma_{i j} \dot{\varepsilon}_{i j}^{p} d V\right)+\rho \frac{\partial f}{\partial \sigma_{i j}}
$$

O problema dual é colocado assim:

$$
\begin{array}{cl}
\text { maximizar } & \mathcal{L}\left(\sigma_{i j}, \mu, \rho\right) \\
\text { sujeito a: } & \frac{\partial \mathcal{L}\left(\sigma_{i j}, \mu, \rho\right)}{\partial \sigma_{i j}}=0 \\
& \rho \geq 0 \\
& \mu \geq 0
\end{array}
$$

Os multiplicadores de Lagrange têm um significado físico importante no problema estudado; os multiplicadores associados ás restrições de igualdade são as taxas de deformação nodais no colapso, que permitem identificar o mecanismo de colapso da estrutura, e aqueles associados ás restrições de desigualdade são os fatores de plastificação [25].

Conhecendo-se a solução do problema primal é possível obter a solução do problema dual e vice-versa, essa propriedade é aproveitada neste trabalho para obter as velocidades nodais que definem o mecanismo de colapso da estrutura geotécnica. Para garantir que o problema de otimização da análise limite tenha um ponto crítico, tem que satisfazer as condições de primeira e segunda ordem ou condições necessárias também conhecidas como condições de Karush-Kuhn-Tucker (KKT), como o problema da AL é convexo as condições KKT são necessariamente suficientes [27]. 


\subsubsection{Algoritmos de otimização}

Uma vez definida a formulação para estimar a carga de colapso (cinemática, estática ou mista) e discretizado o contínuo mediante elementos finitos, é necessário um algoritmo efetivo e eficiente para solucionar o problema estabelecido ao menor custo computacional possível.

Os primeiros algoritmos aplicados à $\mathrm{AL}$ foram desenvolvidos com programação linear (PL); Anderheggen e Knopfel [4], Pastor [2], Sloan [38], Pastor e Turgeman [3], Sloan [5], Sloan e Kleeman [6]. Tendo em geral a seguinte forma (notação matricial):

$$
\begin{aligned}
\text { minimizar } & c^{T} x \\
\text { sujeito } a: & A x=b \\
& B x \leq d
\end{aligned}
$$

em que $x$ é o vetor das variáveis do projeto, a restrição de igualdade representa as equações de equilíbrio e a restrição de desigualdade leva conta do critério de escoamento. Devido a que a maioria dos critérios de escoamento se apresentam de forma não linear, uma linearização de eles deve ser feita antes de colocar a análise como problema de PL [48]. A principal desvantagem de este tipo de programação é o tempo de processamento requerido para solucionar problemas de grande escala.

Devido às dificuldades apresentadas com o tratamento do critério de escoamento alternativamente Zouain et al [49], Lyamin e Sloan [7, 50] e Krabbenhøft e Damkilde [8] fizeram uso de programação não linear (PNL) que permite a inclusão direta de restrições não lineares no processo de otimização, baseadas no algoritmo Quase-Newton para resolver problemas 2D e 3D, porém tendo que suavizar as superfícies definidas pelos critérios com singularidades, o que produz uma redução da acurácia e eficiência de processamento [11].

Karmakar (1984) propôs um novo algoritmo, relacionado com as técnicas de minimização sequencial sem restrição propostas previamente por Fiacco and McCormick (1968), com a vantagem de ser igualmente aplicáveis a programação linear e não linear [48]. Nesses algoritmos a novidade era como a solução evoluía em direção ao ótimo, pelo interior da região factível. Para isso, adiciona-se à função objetivo uma função barreira cuja finalidade é retornar valores tendendo ao infinito a medida que as variáveis se aproxi- 
man da fronteira [23]. Denominados como métodos dos pontos interiores, podem ser aplicados a problemas com a seguinte forma :

$$
\begin{aligned}
\text { minimizar } & f(x) \\
\text { sujeito } a: & A x=b \\
& g_{i}(x) \leq 0 \quad i=1,2, \ldots, n
\end{aligned}
$$

onde as funções $f(x)$ e $g(x)$ geralmente são assumidas convexas.

Ainda mais recente é a aplicação do algoritmo de pontos interiores para lidar com critérios de escoamento na forma cônica, nos quais o problema de otimização é colocado como:

$$
\begin{array}{ll}
\text { minimizar } & c^{T} x \\
\text { sujeito } a: & A x=b \\
& x \in \mathcal{K}
\end{array}
$$

onde x é o vetor das variáveis do problema, $A x=B$ é um conjunto de restrições lineares e $\mathcal{K}$ é um cone convexo associado ao problema. Entre os subcampos da programação cônica os de maior interesse no modelamento de estruturas geotécnicas são a programação cônica de segunda ordem (SOCP) e a programação semi-definida (SDP).

A diferença entre os subcampos da programação cônica vem dada pelo tipo de cone associado ao problema. Nos problemas de SOCP o cone associado é denominado cone de segunda ordem, também conhecido como cone de Lorentz, definido como:

$$
\mathcal{K}_{q}: x 1 \geq \sqrt{x_{2}^{2}+x_{3}^{2}+\cdots+x_{n}^{2}}
$$

Nos problemas de SDP os cones estão formados por um conjunto de matrizes positivas semi-definidas (autovalores não negativos):

$$
\mathcal{K}_{p}: X=\left[\begin{array}{ccc}
x_{11} & \cdots & x_{1 n} \\
\vdots & \ddots & \vdots \\
x_{n 1} & \cdots & x_{n n}
\end{array}\right] \succeq 0
$$

O problema dual correspondente ao problema de otimização cônica vem dado como: 


$$
\begin{aligned}
\text { maximizar } & b^{T} y \\
\text { sujeitoa: } & A^{T} y+s=c \\
& s \in \mathcal{K}
\end{aligned}
$$

Neste trabalho, o problema de otimização foi colocado como SOCP (critério de escoamento D-P) e SDP (critério de escoamento $\mathrm{M}-\mathrm{C}$ ) e resolvido mediante o software MOSEK [51] que faz uso do método primal-dual de pontos interiores proposto por Andersen et al [52]. 


\section{3 \\ IMPLEMENTAÇÃO NUMÉRICA}

Este capítulo abordará a formulação numérica baseada no método dos elementos finitos para problemas de estabilidade axissimétricos na engenharia geotécnica. O emprego do método dos elementos finitos (MEF) para a solução de problemas de estabilidade confere ao método da análise limite um potencial de aplicação a problemas de considerável complexidade (geometria e carregamento). No MEF as variáveis de interesse e as propriedades são definidas no interior de sub-regiões (elementos), a partir da interpolação de seus valores nodais. Na formulação empregada neste trabalho vão se considerar velocidades variando bilinearmente e tensões constantes no interior de cada elemento.

Inicialmente serão revistos conceitos fundamentais do estado axissimétrico, também será estudado o tipo de elemento empregado na discretização do meio contínuo, posteriormente será formulada a equação de equilíbrio e apresentado o procedimento de transformação dos critérios de escoamento ao espaço cônico, finalmente serão apresentadas algumas considerações repeito às condições de contorno, determinação do fator de segurança e obtenção do mecanismo de colapso. Este capítulo vai focar-se exclusivamente no estado axissimétrico, para as comparações feitas com analises tridimensionais foram consultadas as formulações numéricas presentes nos trabalhos de Chahua [25], Camargo [23] e Garcia [27].

\subsection{Estado axissimétrico}

Um corpo no estado axissimétrico, é aquele que apresenta simetria em relação a um eixo, em quanto à geometria, o carregamento, e nas propriedades e distribuição dos materiais. Neste estado o corpo tridimensional se pode considerar como um solido de revolução formado pela rotação completa de uma seção longitudinal bidimensional ao redor do eixo de axissimetria, que em muitos problemas, devido a sua natureza gravítica, é assumido vertical.

Em problemas axissimétricos é comum usar coordenadas cilíndricas, $\mathbf{r}$ (na direção radial), $\mathbf{z}$ (na direção vertical) e $\boldsymbol{\theta}$ (na direção circunferencial). 
Devido à simetria não são gerados deslocamentos na direção circunferencial e os deslocamentos na direção radial e vertical são independentes de $\boldsymbol{\theta}$, temse 4 componentes de deformação [53]:

$$
\varepsilon_{r r}=\frac{\partial u}{\partial r} ; \varepsilon_{z z}=\frac{\partial w}{\partial z} ; \varepsilon_{\theta \theta}=\frac{u}{r} ; \gamma_{r z}=\frac{\partial_{w}}{\partial_{r}}+\frac{\partial_{u}}{\partial_{z}} ; \gamma_{r \theta}=\gamma_{z \theta}=0
$$

Como foi revisto no capítulo 2, na AL são empregadas taxas de deformação $\left(\varepsilon_{i j}\right)$ ao invés de deformações. Para a condição axissimétrica tem-se também 4 componentes de tensão; $\sigma_{r r}, \sigma_{z z}, \sigma_{\theta \theta}$ e $\tau_{r z}$ (Figura 3.1).

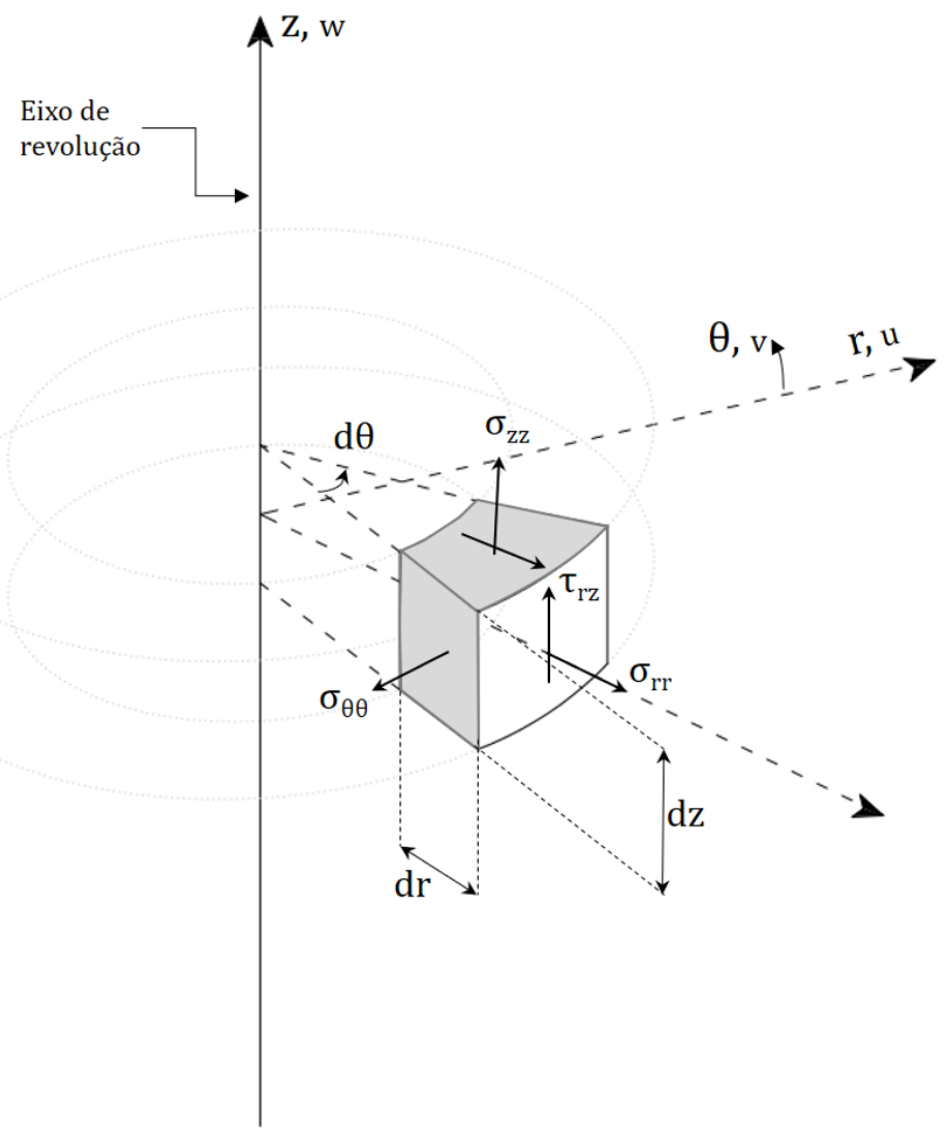

Figura 3.1: Tensões atuantes em um elemento diferencial na condição axissimétrica

\subsection{Discretização do meio contínuo}

\subsubsection{Discretização espacial}

Na discretização do meio contínuo foram utilizados elementos quadrilaterais bidimensionais de 4 nós (Q4), com a interpolação do campo de velocidades bilinear e do campo de tensões constante no interior do elemento. 
Em [46] se verificou que analises feitos com elementos quadrilaterais considerando o campo de tensões constante e variação bilinear das velocidades, geram melhores resultados do que elementos com tensões variando bilinearmente, desde então vem sendo empregados na formulação de problemas de analise limite numérico, segundo o teorema do limite inferior em $[24,25,28]$.

Um elemento finito é dito isoparamétrico quando as mesmas funções de interpolação são empregadas para interpolar tanto as grandezas cinemáticas quanto as grandezas geométricas. No elemento tipo Q4, as funções de interpolação em termos das coordenadas locais $(\xi, \zeta)$ (Figura 3.2), podem ser obtidas de uma maneira simples a partir do produto direto de 2 funções de interpolação 1D, assim :

$$
N_{i}(\xi, \zeta)=\frac{1}{4}\left(1+\xi \xi_{i}\right)\left(1+\zeta \zeta_{i}\right)
$$

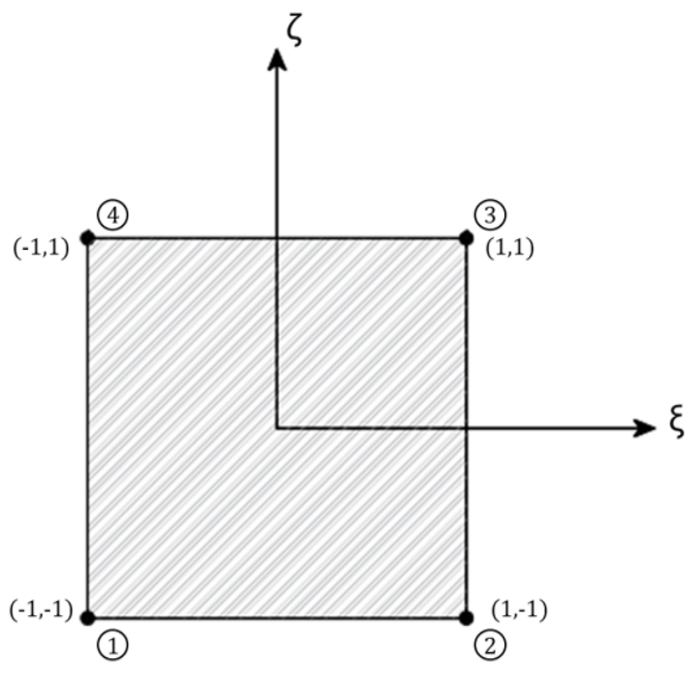

Figura 3.2: Elemento finito quadrilateral isoparamétrico de 4 nós (Q4), em coordenadas locais

sendo $\xi_{i}$ e $\zeta_{i}$ as coordenadas locais do nó $i$, obtendo as seguintes funções de interpolação para cada nó do elemento Q4:

$$
\begin{aligned}
& N_{1}(\xi, \zeta)=\frac{1}{4}(1-\xi)(1-\zeta) \\
& N_{2}(\xi, \zeta)=\frac{1}{4}(1+\xi)(1-\zeta) \\
& N_{3}(\xi, \zeta)=\frac{1}{4}(1+\xi)(1+\zeta) \\
& N_{4}(\xi, \zeta)=\frac{1}{4}(1-\xi)(1+\zeta)
\end{aligned}
$$


Na Figura 3.3 é representada a função de interpolação do nó 1, dada pela equação (3-3). A principal vantagem da formulação de elementos isoparamétricos é que as equações do elemento somente precisam ser avaliadas no sistema de coordenadas do elemento, e posteriormente os resultados são mapeados para o elemento real (Figura 3.4).

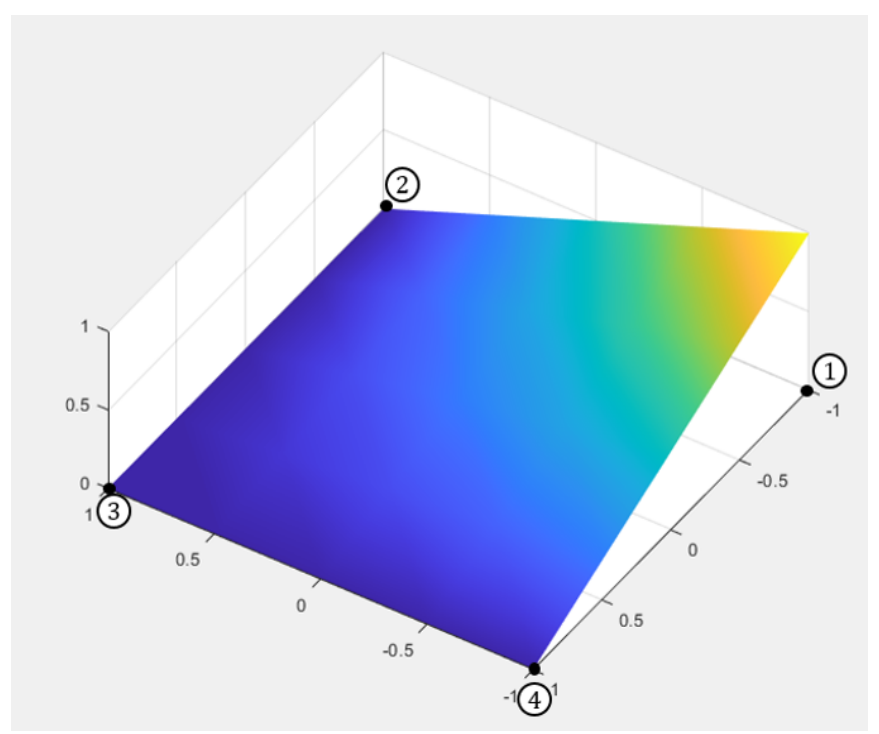

Figura 3.3: Representação gráfica da função de interpolação do nó 1 de um elemento bilinear tipo Q4

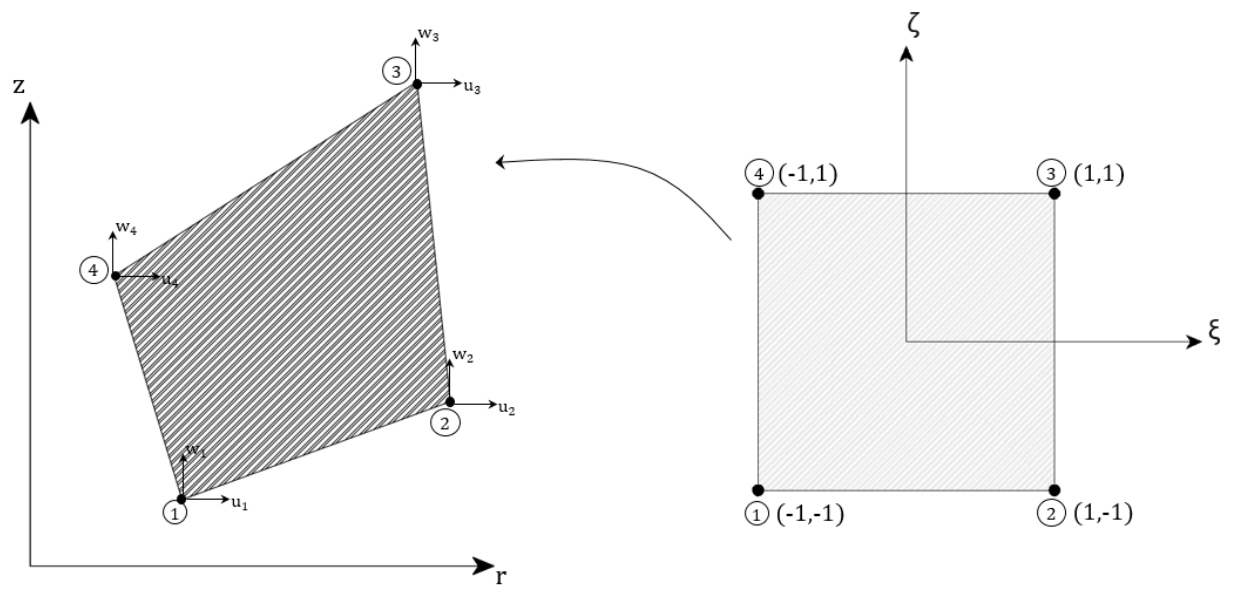

(a)

(b)

Figura 3.4: Mapeamento do elemento mestre (b) para o elemento real (a).

A interpolação das velocidades nodais vem dada como:

$$
\{\dot{u}\}=[N]\{\hat{\dot{u}}\}
$$


onde $\{\dot{u}\}$ é o vetor de velocidades, $[N]$ a matriz de interpolação de velocidades e $\{\hat{\dot{u}}\}$ é o vetor de velocidades nodais:

$$
\left\{\begin{array}{l}
\dot{u} \\
\dot{w}
\end{array}\right\}=\left[\begin{array}{cccccccc}
N_{1} & 0 & N_{2} & 0 & N_{3} & 0 & N_{4} & 0 \\
0 & N_{1} & 0 & N_{2} & 0 & N_{3} & 0 & N_{4}
\end{array}\right]\left\{\begin{array}{c}
\dot{u}_{1} \\
\dot{w}_{1} \\
\dot{u_{2}} \\
\dot{w}_{2} \\
\dot{u} \\
\dot{w_{3}} \\
\dot{u}_{4} \\
\dot{w}_{4}
\end{array}\right\}
$$

A interpolação do raio do elemento $(r)$ é feita a partir dos valores nodais:

$$
r(\xi, \zeta)=\sum_{i=1}^{4} N_{i} \cdot r_{i}=\left\{\begin{array}{llll}
N_{1} & N_{2} & N_{3} & N_{4}
\end{array}\right\}\left\{\begin{array}{l}
r_{1} \\
r_{2} \\
r_{3} \\
r_{4}
\end{array}\right\}
$$

\subsubsection{Transformação de coordenadas}

Para a transformação de coordenadas, necessária no cálculo das derivadas parciais e das integrais de volume da formulação de elementos finitos, é utilizada a matriz Jacobiana. Para isto é preciso estabelecer funções de forma, no caso de elementos isoparamétricos as funções de forma são as mesmas funções de interpolação definidas anteriormente (da equação (3-3) à equação (3-6)). Assim pela regra da cadeia do cálculo diferencial pode-se estabelecer a seguintes relações:

$$
\begin{aligned}
& \frac{\partial N_{i}}{\partial \xi}=\frac{\partial N_{i}}{\partial r} \frac{\partial r}{\partial \xi}+\frac{\partial N_{i}}{\partial z} \frac{\partial z}{\partial \xi} \quad i=1,2,3,4 . \\
& \frac{\partial N_{i}}{\partial \zeta}=\frac{\partial N_{i}}{\partial r} \frac{\partial r}{\partial \zeta}+\frac{\partial N_{i}}{\partial z} \frac{\partial z}{\partial \zeta}
\end{aligned}
$$

Rearranjando sob forma matricial as equações (3-10) e (3-11), tem-se:

$$
\left\{\begin{array}{c}
\frac{\partial N_{i}}{\partial \xi} \\
\frac{\partial N_{i}}{\partial \zeta}
\end{array}\right\}=\left[\begin{array}{ll}
\frac{\partial r}{\partial \xi} & \frac{\partial z}{\partial \xi} \\
\frac{\partial r}{\partial \zeta} & \frac{\partial z}{\partial \zeta}
\end{array}\right]\left\{\begin{array}{c}
\frac{\partial N_{i}}{\partial r} \\
\frac{\partial N_{i}}{\partial z}
\end{array}\right\}
$$




$$
\left\{\begin{array}{c}
\frac{\partial N_{i}}{\partial \xi} \\
\frac{\partial N_{i}}{\partial \zeta}
\end{array}\right\}=[J]\left\{\begin{array}{c}
\frac{\partial N_{i}}{\partial r} \\
\frac{\partial N_{i}}{\partial z}
\end{array}\right\}
$$

Na equação (3-12) o vetor do lado esquerdo e a matriz do lado direito, matriz Jacobiana $[J]$, são conhecidos já que as funções de interpolação são definidas considerando o sistema de coordenadas local e o mapeamento é isoparamétrico, por outro lado o vetor do lado direito é o vetor procurado, isolando-o tem-se:

$$
\left\{\begin{array}{c}
\frac{\partial N_{i}}{\partial r} \\
\frac{\partial N_{i}}{\partial z}
\end{array}\right\}=[J]^{-1}\left\{\begin{array}{c}
\frac{\partial N_{i}}{\partial \xi} \\
\frac{\partial N_{i}}{\partial \zeta}
\end{array}\right\}
$$

onde

$$
[J]^{-1}=[\Gamma] \longrightarrow \frac{1}{|J|}\left[\begin{array}{cc}
J_{22} & -J_{12} \\
-J_{21} & J_{11}
\end{array}\right]=\left[\begin{array}{ll}
\Gamma_{11} & \Gamma_{12} \\
\Gamma_{21} & \Gamma_{22}
\end{array}\right]
$$

\subsubsection{Integração numérica}

Por praticidade e eficiência, as integrais resultantes da formulação por elementos finitos são avaliadas por meio da quadratura de Gauss-Legendre:

$$
\int_{-1}^{1} f(x) d x \cong \sum_{i=1}^{n} W_{i} f\left(x_{i}\right)
$$

onde $f(x)$ é a função cuja integral se deseja calcular, $x_{i}$ são os pontos onde o valor da função é avaliada, $n$ representa a quantidade desses pontos e $W_{i}$ são os pesos de ponderação. Neste trabalho para o elemento Q4, foram considerados 4 pontos de integração ( 2 pontos em cada direção), na Tabela 3.1 são especificadas as coordenadas paramétricas ou locais desses pontos e seus respectivos fatores de ponderação ou pesos.

Tabela 3.1: Coordenadas locais dos pontos de integração e seus respectivos fatores de ponderação

\begin{tabular}{ccccc}
\hline & \multicolumn{2}{c}{$\begin{array}{c}\text { Coordenadas } \\
\text { pontos de integração }\end{array}$} & \multicolumn{2}{c}{ Pesos } \\
\cline { 2 - 5 } & $\xi_{i}$ & $\zeta_{i}$ & $W_{\xi_{i}}$ & $W_{\zeta_{i}}$ \\
\hline 1 & $-1 / \sqrt{3}$ & $-1 / \sqrt{3}$ & 1 & 1 \\
2 & $1 / \sqrt{3}$ & $-1 / \sqrt{3}$ & 1 & 1 \\
3 & $1 / \sqrt{3}$ & $1 / \sqrt{3}$ & 1 & 1 \\
4 & $-1 / \sqrt{3}$ & $1 / \sqrt{3}$ & 1 & 1 \\
\hline
\end{tabular}


3.3 Formulação da equação de equilíbrio (restrição de igualdade) do problema de otimização

Para a formulação da restrição de igualdade na ALN, nesta secção são estudadas previamente as seguintes matrizes e vetores que a compõem:

\subsubsection{Matriz de compatibilidade cinemática}

Para um elemento finito a relação entre as taxas de deformação e as velocidades, vem dada como:

$$
\left\{\begin{array}{c}
\dot{\varepsilon_{r r}} \\
\dot{\varepsilon_{z z}} \\
\dot{\gamma_{r z}} \\
\dot{\varepsilon_{\theta \theta}}
\end{array}\right\}=\left[\begin{array}{cc}
\frac{\partial}{\partial r} & 0 \\
0 & \frac{\partial}{\partial z} \\
\frac{\partial}{\partial z} & \frac{\partial}{\partial r} \\
\frac{1}{r} & 0
\end{array}\right]\left\{\begin{array}{l}
\dot{u} \\
\dot{w}
\end{array}\right\}
$$

A equação (3-17) pode-se rescrever convenientemente como:

$$
\left\{\begin{array}{l}
\dot{\varepsilon_{r r}} \\
\dot{\varepsilon_{z z}} \\
\dot{\gamma_{r z}} \\
\dot{\varepsilon_{\theta \theta}}
\end{array}\right\}=\left[\begin{array}{ccccc}
1 & 0 & 0 & 0 & 0 \\
0 & 0 & 0 & 1 & 0 \\
0 & 1 & 1 & 0 & 0 \\
0 & 0 & 0 & 0 & \frac{1}{r}
\end{array}\right]\left\{\begin{array}{c}
\frac{\partial \dot{u}}{\partial r} \\
\frac{\partial \dot{u}}{\partial z} \\
\frac{\partial \dot{w}}{\partial r} \\
\frac{\partial \dot{w}}{\partial z} \\
\dot{u}
\end{array}\right\}
$$

com

$$
[H]=\left[\begin{array}{ccccc}
1 & 0 & 0 & 0 & 0 \\
0 & 0 & 0 & 1 & 0 \\
0 & 1 & 1 & 0 & 0 \\
0 & 0 & 0 & 0 & \frac{1}{r}
\end{array}\right]
$$

Na equação (3-18) o vetor que contém as derivadas parciais das velocidades respeito ao sistema de coordenadas globais $(r, z)$ é obtido indiretamente a partir do vetor com as derivadas parciais das velocidades respeito ao sistema de coordenadas locais $(\xi, \zeta)$, para isto é necessário fazer uma transformação de coordenadas com a matriz $\Gamma$ (equação (3-15)). 
com

$$
\left\{\begin{array}{c}
\frac{\partial \dot{u}}{\partial r} \\
\frac{\partial \dot{u}}{\partial z} \\
\frac{\partial \dot{w}}{\partial r} \\
\frac{\partial \dot{w}}{\partial z} \\
\dot{u}
\end{array}\right\}=\left[\begin{array}{ccccc}
\Gamma_{11} & \Gamma_{12} & 0 & 0 & 0 \\
\Gamma_{21} & \Gamma_{22} & 0 & 0 & 0 \\
0 & 0 & \Gamma_{11} & \Gamma_{12} & 0 \\
0 & 0 & \Gamma_{21} & \Gamma_{22} & 0 \\
0 & 0 & 0 & 0 & 1
\end{array}\right\}\left\{\begin{array}{c}
\frac{\partial \dot{u}}{\partial \xi} \\
\frac{\partial \dot{u}}{\partial \zeta} \\
\frac{\partial \dot{w}}{\partial \xi} \\
\frac{\partial \dot{w}}{\partial \zeta} \\
\dot{u}
\end{array}\right\}
$$

$$
\left[\Gamma_{\text {axis }}\right]=\left[\begin{array}{ccccc}
\Gamma_{11} & \Gamma_{12} & 0 & 0 & 0 \\
\Gamma_{21} & \Gamma_{22} & 0 & 0 & 0 \\
0 & 0 & \Gamma_{11} & \Gamma_{12} & 0 \\
0 & 0 & \Gamma_{21} & \Gamma_{22} & 0 \\
0 & 0 & 0 & 0 & 1
\end{array}\right]
$$

Para obter o vetor do lado direito da equação (3-20) (com as derivadas parciais das velocidades respeito ao sistema de coordenadas locais), o processo envolve a obtenção do gradiente das funções de interpolação do elemento, resultando na seguinte expressão:

$$
\left\{\begin{array}{c}
\frac{\partial \dot{u}}{\partial \xi} \\
\frac{\partial \dot{u}}{\partial \zeta} \\
\frac{\partial \dot{w}}{\partial \xi} \\
\frac{\partial \dot{w}}{\partial \zeta} \\
\dot{u}
\end{array}\right\}=\left[\begin{array}{cccccccc}
\frac{\partial N_{1}}{\partial \xi} & 0 & \frac{\partial N_{2}}{\partial \xi} & 0 & \frac{\partial N_{3}}{\partial \xi} & 0 & \frac{\partial N_{4}}{\partial \xi} & 0 \\
\frac{\partial N_{1}}{\partial \zeta} & 0 & \frac{\partial N_{2}}{\partial \zeta} & 0 & \frac{\partial N_{3}}{\partial \zeta} & 0 & \frac{\partial N_{4}}{\partial \zeta} & 0 \\
0 & \frac{\partial N_{1}}{\partial \xi} & 0 & \frac{\partial N_{2}}{\partial \xi} & 0 & \frac{\partial N_{3}}{\partial \xi} & 0 & \frac{\partial N_{4}}{\partial \xi} \\
0 & \frac{\partial N_{1}}{\partial \zeta} & 0 & \frac{\partial N_{2}}{\partial \zeta} & 0 & \frac{\partial N_{3}}{\partial \zeta} & 0 & \frac{\partial N_{4}}{\partial \zeta} \\
N_{1} & 0 & N_{2} & 0 & N_{3} & 0 & N_{4} & 0
\end{array}\right]\left\{\begin{array}{c}
\dot{u}_{1} \\
\dot{w}_{1} \\
\dot{u}_{2} \\
\dot{w}_{2} \\
\dot{u}_{3} \\
\dot{w}_{3} \\
\dot{u}_{4} \\
\dot{w}_{4}
\end{array}\right\}
$$

com:

$$
[D n d]=\left[\begin{array}{cccccccc}
\frac{\partial N_{1}}{\partial \xi} & 0 & \frac{\partial N_{2}}{\partial \xi} & 0 & \frac{\partial N_{3}}{\partial \xi} & 0 & \frac{\partial N_{4}}{\partial \xi} & 0 \\
\frac{\partial N_{1}}{\partial \zeta} & 0 & \frac{\partial N_{2}}{\partial \zeta} & 0 & \frac{\partial N_{3}}{\partial \zeta} & 0 & \frac{\partial N_{4}}{\partial \zeta} & 0 \\
0 & \frac{\partial N_{1}}{\partial \xi} & 0 & \frac{\partial N_{2}}{\partial \xi} & 0 & \frac{\partial N_{3}}{\partial \xi} & 0 & \frac{\partial N_{4}}{\partial \xi} \\
0 & \frac{\partial N_{1}}{\partial \zeta} & 0 & \frac{\partial N_{2}}{\partial \zeta} & 0 & \frac{\partial N_{3}}{\partial \zeta} & 0 & \frac{\partial N_{4}}{\partial \zeta} \\
N_{1} & 0 & N_{2} & 0 & N_{3} & 0 & N_{4} & 0
\end{array}\right]
$$

Substituindo a equação (3-22) na equação (3-20) e posteriormente a 
equação (3-20) na equação (3-18), se pode obter uma expressão que permite estimar as taxas de deformação de um elemento finito Q4 a partir de suas velocidades nodais:

$$
\{\dot{\varepsilon}\}=\left[B_{e}\right]\{\hat{\dot{u}}\}
$$

onde $\{\dot{\varepsilon}\}$ é o vetor das taxas de deformação, $\{\hat{\dot{u}}\}$ é o vetor das velocidades nodais e $\left[B_{e}\right]$ é a matriz de compatibilidade cinemática, calculada como o produto das matrizes das equações (3-19), (3-21) e (3-23):

$$
\left[B_{e}\right]=[H] \times\left[\Gamma_{\text {axis }}\right] \times[D n d]
$$

\subsubsection{Matriz de Equilíbrio}

Para um elemento finito a matriz de equilíbrio $\left[G_{e}\right]$ vem definida como:

$$
\left[G_{e}\right]=\int_{V}\left[B_{e}\right]^{T} d V=\int_{A} \int_{0}^{2 \pi}\left[B_{e}\right]^{T} r d \theta d r d z=2 \pi \int_{A}\left[B_{e}\right]^{T} r d r d z
$$

a integral da equação (3-26) é avaliada no sistema de coordenadas locais, para isto é necessária uma transformação de coordenadas feita por meio do determinante da matriz Jacobiana, conhecido como Jacobiano $(|J|)$.

$$
\left[G_{e}\right]=2 \pi \int_{A}\left[B_{e}\right]^{T} r d r d z \stackrel{\text { transformação }}{\longrightarrow}\left[G_{e}\right]=2 \pi \int_{-1}^{+1} \int_{-1}^{+1}\left[B_{e}\right]^{T} r|J| d \xi d \zeta
$$

Pela quadratura Gauss-Legendre, a integral da equação (3-27) é calculada como:

$$
\left[G_{e}\right] \cong 2 \pi \sum_{i=1}^{2} \sum_{j=1}^{2} W_{\xi_{i}} W_{\zeta_{j}}\left[B_{e}\right]^{T} r|J|
$$

\subsubsection{Vetores de cargas nodais}

$\mathrm{O}$ vetor de força de volume para um elemento finito, vem dado pela seguinte integral:

$$
\left\{f_{e}\right\}=\int_{V}[N]^{T}\{F\} d V=\int_{A} \int_{0}^{2 \pi}[N]^{T}\{F\} r d \theta d A=2 \pi \int_{A}[N]^{T}\{F\} r d r d z
$$

com

$$
F=\left\{F_{r}, F_{z}\right\}^{T}
$$


fazendo a transformação de coordenadas tem-se

$$
\left\{f_{e}\right\}=2 \pi \int_{A}[N]^{T}\{F\} r d r d z \stackrel{\text { transformação }}{\longrightarrow}\left\{f_{e}\right\}=2 \pi \int_{-1}^{+1} \int_{-1}^{+1}[N]^{T}\{F\} r|J| d \xi d \zeta
$$

a integral é resolvida numericamente como:

$$
\left\{f_{e}\right\}=2 \pi \sum_{i=1}^{2} \sum_{j=1}^{2} W_{\xi_{i}} W_{\zeta_{j}}[N]^{T}\{F\} r|J|
$$

Para o caso das forças de superfície, o seu respectivo vetor é obtido da avaliação numérica ou analítica da seguinte integral:

$$
\left\{t_{e}\right\}=\int_{0}^{2 \pi} \int_{l}[N]^{T}\{T\} r d l d \theta=2 \pi \int_{l}[N]^{T}\{T\} r d l
$$

com

$$
T=\left\{T_{r}, T_{z}\right\}^{T}
$$

\subsubsection{Formulação matricial da equação de equilíbrio}

A restrição de igualdade da equação (2-14), pode-se reescrever na forma matricial como:

$$
\begin{aligned}
\int_{V}\{\delta \dot{\varepsilon}\}^{T}\left\{\sigma_{e}\right\} d V= & \int_{V}\{\delta \dot{u}\}^{T}\left\{F_{o}\right\} d V+\int_{A}\{\delta \dot{u}\}^{T}\left\{T_{o}\right\} d A \\
& +\lambda\left(\int_{V}\{\delta \dot{u}\}^{T}\{F\} d V+\int_{A}\{\delta \dot{u}\}^{T}\{T\} d A\right)
\end{aligned}
$$

onde $\{\delta \dot{\varepsilon}\}$ é o vetor com as taxas de deformação, $\left\{\sigma_{e}\right\}$ é o vetor com as tensões, $\{\delta \dot{u}\}$ é o vetor com as velocidades, $\left\{F_{o}\right\}$ e $\left\{T_{o}\right\}$ são os vetores de forças de volume e de superfície, respectivamente, que ficam constantes e $\{F\}$ e $\{T\}$ os vetores de forças que são multiplicados pelo fator de colapso $\lambda$. Considerando a discretização do meio contínuo em elementos finitos e incluindo na equação (3-35) as matrizes estudadas na secções 3.3.1, 3.3 .2 e 3.3.3, a equação (3-35) se pode reescrever, para um elemento finito, como:

$$
\begin{aligned}
\int_{V}\{\delta \hat{\dot{u}}\}^{T}\left[B_{e}\right]^{T}\left\{\sigma_{e}\right\} d V= & \int_{V}\{\delta \hat{\dot{u}}\}^{T}[N]^{T}\left\{F_{o}\right\} d V+\int_{A}\{\delta \hat{\dot{u}}\}^{T}[N]^{T}\left\{T_{o}\right\} d A \\
& +\lambda\left(\int_{V}\{\delta \hat{\dot{u}}\}^{T}[N]^{T}\{F\} d V+\int_{A}\{\delta \hat{\dot{u}}\}^{T}[N]^{T}\{T\} d A\right)
\end{aligned}
$$


Simplificando a equação (3-36), considerando um valor arbitrário das velocidades nodais $\{\delta \hat{\dot{u}}\}$, tem-se:

$$
\begin{aligned}
\int_{V}\left[B_{e}\right]^{T}\left\{\sigma_{e}\right\} d V= & \int_{V}[N]^{T}\left\{F_{o}\right\} d V+\int_{A}[N]^{T}\left\{T_{o}\right\} d A \\
& +\lambda\left(\int_{V}[N]^{T}\{F\} d V+\int_{A}[N]^{T}\{T\} d A\right)
\end{aligned}
$$

Finalmente a equação (3-37) incluindo as definições de matriz de equilíbrio do elemento $\left(\left[G_{e}\right]\right)$ e vetores de cargas nodais $\left(\left\{f_{e}\right\}\right.$ e $\left.\left\{t_{e}\right\}\right)$, pode ser expressa como:

$$
\left[G_{e}\right]\left\{\sigma_{e}\right\}=\left\{f_{e o}\right\}+\left\{t_{e o}\right\}+\lambda\left(\left\{f_{e}\right\}+\left\{t_{e}\right\}\right)
$$

Na equação (3-38), referente a um elemento finito, a matriz de equilíbrio $\left(\left[G_{e}\right]\right)$ tem dimensões $8 \times 4$, o vetor de tensões $\left\{\sigma_{e}\right\}$ tem dimensões $4 \times 1$, e os vetores de cargas nodais constantes $\left(\left\{f_{e o}\right\} e\left\{t_{e o}\right\}\right)$ e variáveis $\left(\left\{f_{e}\right\} e\left\{t_{e}\right\}\right)$ têm dimensões $8 \times 1$.

Para obter a equação de equilíbrio global da estrutura geotécnica, é feito um acoplamento das matrizes elementares, sendo $N_{E}$ o número total de elementos e $N_{N}$ o número de nós do domínio, a matriz global de equilíbrio $[G]$ terá dimensões $2 N_{N} \times 4 N_{E}$, o vetor global de tensões $\{\sigma\}$ terá dimensões $4 N_{E} \times 1$, e os vetores globais de cargas nodais $\left(\left\{f_{o}\right\},\left\{t_{o}\right\},\{f\} e\{t\}\right)$ terão dimensões $2 N_{N} \times 1$, resultado na seguinte equação:

$$
[G]\{\sigma\}=\left\{f_{o}\right\}+\left\{t_{o}\right\}+\lambda(\{f\}+\{t\})
$$

\subsection{Transformação dos critérios de escoamento ao espaço cônico}

Em seguida é apresentado o procedimento para a implementação dos critérios D-P e M-C no problema de otimização cônica, para o estado axissimétrico. Os criterios de escoamento foram adaptados com a finalidade de utilizar o software Mosek Aps 9.2 [51].

\subsubsection{Critério de escoamento de Drucker-Prager na forma Cônica de Segunda Ordem, sem poropressões}

Inicialmente a transformação do critério D-P no estado tridimensional para o espaço cônico de segunda ordem foi apresentada por Makrodimopoulos e Martin [10], sendo empregada também nos trabalhos realizados na linha de pesquisa de ALN da PUC-RIO (Camargo [23] e García [27]). A 
transformação do critério no estado axissimétrico, resulta em uma simplificação dos trabalhos acima mencionados. Partido da equação (2-8) com:

$$
\alpha I_{1}^{\prime}+\sqrt{J_{2}}-k \leq 0 \rightarrow\left\{\begin{array}{l}
I_{1}^{\prime}=3 \cdot \sigma_{m}=\sigma_{r r}+\sigma_{z z}+\sigma_{\theta \theta} \\
J_{2}=\frac{1}{2}\left(S_{r r}^{2}+S_{z z}^{2}+S_{\theta \theta}^{2}\right)+S_{r z}^{2}
\end{array}\right.
$$

onde $S_{r r}, S_{z z}, S_{\theta \theta}$ e $S_{r z}$ são componentes do tensor de tensões desviadoras, definidas como:

$$
\left\{\begin{aligned}
S_{r r} & =\sigma_{r r}-\sigma_{m}=\frac{2}{3} \sigma_{r r}-\frac{1}{3} \sigma_{z z}-\frac{1}{3} \sigma_{\theta \theta} \\
S_{z z} & =\sigma_{z z}-\sigma_{m}=\frac{2}{3} \sigma_{z z}-\frac{1}{3} \sigma_{r r}-\frac{1}{3} \sigma_{\theta \theta} \\
S_{\theta \theta} & =\sigma_{\theta \theta}-\sigma_{m}=\frac{2}{3} \sigma_{\theta \theta}-\frac{1}{3} \sigma_{r r}-\frac{1}{3} \sigma_{z z} \\
S_{r z} & =\tau_{r z}
\end{aligned}\right.
$$

A restrição dada pelo critério de escoamento pode-se transformar em uma restrição linear de igualdade acoplada com uma restrição cônica de segundo ordem, mediante a introdução de uma variável auxiliar $z$ :

$$
\left\{\begin{array}{l}
z=k-\alpha I_{1}^{\prime} \\
\sqrt{\rho_{2}^{2}+\rho_{3}^{2}+\rho_{4}^{2}} \leq z
\end{array}\right.
$$

A restrição cônica de segundo ordem neste caso é obtida substituindo as expressões de (3-41) na expressão de (3-40) referente ao segundo invariante de tensões de desvio $J_{2}$ e posteriormente rearranjando os seus termos:

$$
\sqrt{J_{2}}=\sqrt{\left(\frac{1}{2} \sigma_{r r}-\frac{1}{2} \sigma_{\theta \theta}\right)^{2}+\left(-\frac{\sqrt{3}}{6} \sigma_{r r}+\frac{\sqrt{3}}{3} \sigma_{z z}-\frac{\sqrt{3}}{6} \sigma_{\theta \theta}\right)^{2}+\tau_{r z}^{2}}
$$

com

$$
\begin{aligned}
& \rho_{2}=\frac{1}{2} \sigma_{r r}-\frac{1}{2} \sigma_{\theta \theta} \\
& \rho_{3}=-\frac{\sqrt{3}}{6} \sigma_{r r}+\frac{\sqrt{3}}{3} \sigma_{z z}-\frac{\sqrt{3}}{6} \sigma_{\theta \theta} \\
& \rho_{4}=\tau_{r z}
\end{aligned}
$$

Sendo assim, na formulação do problema SOCP as variáveis correspondentes ao vetor das tensões para um elemento $\left\{\sigma_{e}\right\}$ são substituídas por as de um vetor auxiliar $\left\{\rho_{e}\right\}$, que representa o critério de escoamento D-P, matricialmente a transformação do critério é expressa assim: 


$$
\begin{gathered}
\left\{\begin{array}{c}
z \\
\rho_{2} \\
\rho_{3} \\
\rho_{4}
\end{array}\right\}=\left[\begin{array}{cccc}
-\alpha & -\alpha & 0 & -\alpha \\
\frac{1}{2} & 0 & 0 & -\frac{1}{2} \\
-\frac{\sqrt{3}}{6} & \frac{\sqrt{3}}{3} & 0 & -\frac{\sqrt{3}}{6} \\
0 & 0 & 1 & 0
\end{array}\right]\left\{\begin{array}{c}
\sigma_{r r} \\
\sigma_{z z} \\
\tau_{r z} \\
\sigma_{\theta \theta}
\end{array}\right\}+\left\{\begin{array}{l}
k \\
0 \\
0 \\
0
\end{array}\right\} \\
\left\{\rho_{e}\right\}=[D]\left\{\sigma_{e}\right\}+\{d\}
\end{gathered}
$$

onde $[D]$ e $\{d\}$ são a matriz e o vetor de transformação pro espaço cônico respectivamente. Na equação (3-46) isolando o vetor das tensões temse:

$$
\left\{\sigma_{e}\right\}=[D]^{-1}\left\{\rho_{e}\right\}-[D]^{-1}\{d\}
$$

Substituindo a equação (3-47) na equação de equilíbrio (3-38), é feita a transformação para cada elemento da estrutura geotécnica e posteriormente é realizado um acoplamento das matrizes elementares, para definir o problema primal de otimização com SOCP, como:

$$
\begin{aligned}
\text { minimizar } & (-\lambda) \\
\text { sujeito a: } & {\left[G_{\text {conic }}\right]\{\rho\}-\left\{L_{\text {conic }}\right\}=\left\{f_{o}\right\}+\left\{t_{o}\right\}+\lambda(\{f\}+\{t\}) } \\
& \rho \in \kappa
\end{aligned}
$$

onde $\left[G_{\text {conic }}\right]$ resulta do processo de acoplamento das matrizes de equilíbrio elementares no espaço cônico $\left\{G_{e_{\text {conic }}}\right\}$ e $\left\{L_{\text {conic }}\right\}$ e do acoplamento dos vetores $\left\{L_{e_{\text {conic }}}\right\}$, sendo:

$$
\begin{aligned}
& {\left[G_{e_{\text {conic }}}\right]=\left[G_{e}\right] \times[D]^{-1}} \\
& \left\{L_{e_{\text {conic }}}\right\}=\left[G_{e}\right] \times[D]^{-1} \times\{d\}
\end{aligned}
$$

\subsubsection{Critério de escoamento de Drucker-Prager na forma Cônica de Segunda Ordem, considerando poropressões}

A inclusão do efeito da poropressão no critério D-P foi apresentado por Camargo [23], já que o critério de ruptura é formulado em função das tensões efetivas, que mobilizam a resistência ao cisalhamento do solo, as expressões desenvolvidas na secção 3.4.1 requerem uma modificação na definição do primeiro invariante do tensor de tensões $I_{1}^{\prime}\left(J_{2}\right.$ não resulta afetado):

$$
I_{1}^{\prime}=3 \cdot \sigma_{m}^{\prime}=\sigma_{r r}+\sigma_{z z}+\sigma_{\theta \theta}-3 u
$$

Portanto, a variável auxiliar $z$ é definida como: 


$$
z=k-\alpha I_{1}^{\prime}=-\alpha\left(\sigma_{r r}+\sigma_{z z}+\sigma_{\theta \theta}\right)+k+3 \alpha u
$$

Deste modo a equação, na forma matricial, da transformação do problema para SOCP, vem dado como:

$$
\left\{\begin{array}{c}
z \\
\rho_{2} \\
\rho_{3} \\
\rho_{4}
\end{array}\right\}=\left[\begin{array}{cccc}
-\alpha & -\alpha & 0 & -\alpha \\
\frac{1}{2} & 0 & 0 & -\frac{1}{2} \\
-\frac{\sqrt{3}}{6} & \frac{\sqrt{3}}{3} & 0 & -\frac{\sqrt{3}}{6} \\
0 & 0 & 1 & 0
\end{array}\right]\left\{\begin{array}{c}
\sigma_{r r} \\
\sigma_{z z} \\
\tau_{r z} \\
\sigma_{\theta \theta}
\end{array}\right\}+\left\{\begin{array}{c}
k+3 \alpha u \\
0 \\
0 \\
0
\end{array}\right\}
$$

\subsubsection{Critério de escoamento de Mohr-Coulomb na forma Semidefinida}

Em seguida é formulado o critério M-C como SDP, com base nos trabalhos de Krabbenhøft et al. [9], Martin e Makrodimopoulos [54], Garcia [27], Bruno et al. [55] nos quais foi estudada a adaptação do criterio M-C, para problemas tridimensionais e o trabalho mais recente de Sun e Yang [16] para problemas na condição axissimétrica.

Ao contrario da programação SOCP, na qual não se consegue tratar

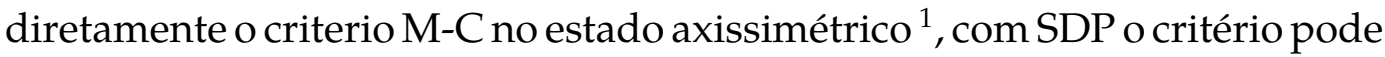
ser tratado em sua forma original e sem precisar fazer suposições sobre $\sigma_{\theta \theta}$.

Inicialmente o vetor de tensões $\{\sigma\}$ é rearranjado de maneira a representar uma matriz simétrica positiva-semidefinida $([\Sigma] \succeq 0)$, mediante a função mat, como é explicado na seguinte expressão:

$$
[\Sigma]=\operatorname{mat}\{\sigma\}
$$

com

$$
[\Sigma]=\left[\begin{array}{ccc}
\sigma_{r r} & \tau_{r z} & 0 \\
\tau_{r z} & \sigma_{z z} & 0 \\
0 & 0 & \sigma_{\theta \theta}
\end{array}\right] \quad\{\sigma\}=\left\{\begin{array}{c}
\sigma_{r r} \\
\sigma_{z z} \\
\tau_{r z} \\
\sigma_{\theta \theta}
\end{array}\right\}
$$

O critério de escoamento M-C representado na equação (2-7) pode-se reescrever como:

$$
\alpha \sigma_{1}-\beta \sigma_{3} \leq \kappa
$$

\footnotetext{
${ }^{1}$ Tem-se trabalhos nos quais o critério M-C é representado por um conjunto de 3 restrições, os possíveis casos nos quais pode-se colocar o critério dependendo da suposição feita respeito a $\sigma_{\theta \theta}$ (Chong et al. [12]), Rahaman et al [56]).
} 
com

$$
\begin{aligned}
\alpha & =1+\operatorname{sen} \phi \\
\beta & =1-\operatorname{sen} \phi \\
\kappa & =2 c \cos \phi
\end{aligned}
$$

Para resolver o problema com SDP o critério pode ser expresso em função de duas restrições semidefinidas:

$$
\left\{\begin{array}{l}
-t[I]-\alpha[\Sigma]+\kappa[I] \succeq 0 \\
t[I]+\beta[\Sigma] \succeq 0
\end{array}\right.
$$

onde $[I]$ é a matriz identidade e $t$ é uma variável auxiliar, incluindo na definição das restrições semidefinidas as matrizes:

$$
[X]=\left[\begin{array}{ccc}
x_{11} & x_{12} & 0 \\
x_{21} & x_{22} & 0 \\
0 & 0 & x_{33}
\end{array}\right] \succeq 0 \quad[Z]=\left[\begin{array}{ccc}
z_{11} & z_{12} & 0 \\
z_{21} & z_{22} & 0 \\
0 & 0 & z_{33}
\end{array}\right] \succeq 0
$$

As inequações de (3-57) são reescritas como:

$$
\left\{\begin{array}{l}
-t[I]-\alpha[\Sigma]+\kappa[I]=[X] \\
t[I]+\beta[\Sigma]=[Z]
\end{array}\right.
$$

Das equações de (3-59) surgem 8 restrições para cada elemento:

$$
\left\{\begin{array}{l}
x_{11}+\alpha \sigma_{r r}+t=\kappa \\
x_{22}+\alpha \sigma_{z z}+t=\kappa \\
x_{33}+\alpha \sigma_{\theta \theta}+t=\kappa \\
x_{12}+\alpha \tau_{r z}=0 \\
z_{11}-\beta \sigma_{r r}-t=0 \\
z_{22}-\beta \sigma_{z z}-t=0 \\
z_{33}-\beta \sigma_{\theta \theta}-t=0 \\
z_{12}-\beta \tau_{r z}=0
\end{array}\right.
$$

O problema primal de otimização com programação SDP resulta definido como:

$$
\begin{aligned}
\text { minimizar } & (-\lambda) \\
\text { sujeito } a: & {[G]\{\sigma\}=\left\{f_{o}\right\}+\left\{t_{o}\right\}+\lambda(\{f\}+\{t\}) } \\
& {[X] \succeq 0 } \\
& {[Z] \succeq 0 }
\end{aligned}
$$




\subsection{Considerações finais da implementação numérica}

\subsubsection{Condições de contorno}

Nos problemas estudados as condições de contorno impostas à estrutura geotécnica foram do primeiro tipo, ou de Dirichlet, com velocidades nulas prescritas com o objetivo de garantir a ausência de movimentos de corpo rígido. Na equação (3-39) são eliminadas as linhas da matriz de equilíbrio global e dos vetores globais de cargas nodais, correspondentes aos graus de liberdade restritos.

\subsubsection{Estimação do fator de segurança}

A estabilidade da estrutura geotécnica pode-se avaliar em termos de uma das duas seguintes questões [57]:

- Uma vez conhecida a resistência do solo, por qual fator deverão ser multiplicadas as cargas externas para causar o incipiente estado de colapso?

- Uma vez conhecidas as cargas externas, por qual fator devera-se reduzir a resistência do solo para causar o incipiente estado de colapso?

A resposta à primeira questão vem dada diretamente da ALN, em termos do fator de colapso $\lambda$, a resposta à segunda pode-se conseguir da ALN incluindo o método de redução de resistência, obtendo o fator de segurança $F S$, processo que resulta mais exigente computacionalmente, mas, cujo resultado é mais relevante na prática.

O FS é obtido via redução dos parâmetros do modelo M-C:

$$
F S=\frac{c}{c_{r}}=\frac{\tan \phi}{\tan \phi_{r}} \rightarrow\left\{\begin{array}{l}
c_{r}=\frac{c}{F R} \\
\phi_{r}=\tan ^{-1}\left(\frac{\tan \phi}{F R}\right)
\end{array}\right.
$$

onde FR é o fator de redução de resistência. Dependendo das cargas maximizadas ou afetadas pelo fator de colapso; peso próprio da estrutura ou força fictícia (como foi feito em [25] e [23], para problemas considerando o efeito da poropressão) o procedimento da obtenção do $F S$ na ALN varia, como é representado na Figura 3.5.

Quando a analise é feita maximizando o peso próprio da estrutura o fator de segurança $F S$ é o fator de redução $F R$ para o qual o fator de colapso $\lambda$ é igual a 1. Quando é considerada a maximização de uma força fictícia (que será empregada posteriormente nos exemplos que consideram um elemento 


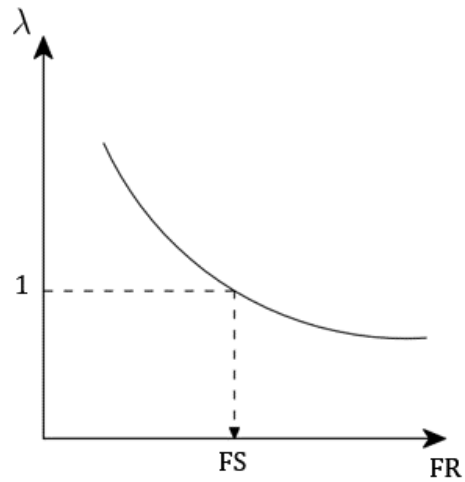

Maximização do peso próprio da estrutura

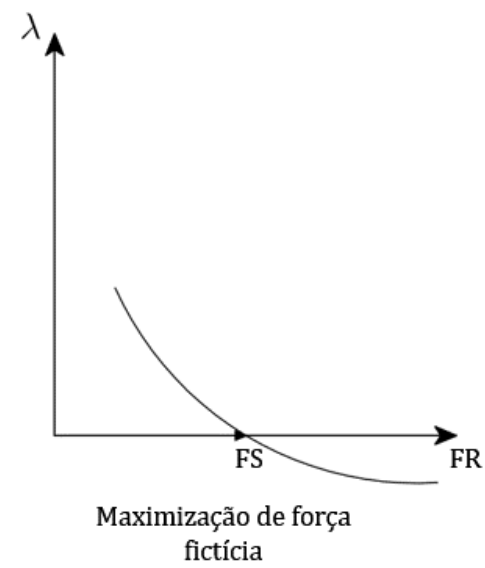

Figura 3.5: Estimação do fator de segurança na ALN

de concreto e o efeito da poropressão, como uma técnica para manter estável o código) o fator de segurança $F S$ é o fator de redução $F R$ para o qual o fator de colapso $\lambda$ é igual a 0 . Com o fim de automatizar o processo iterativo de calculo do FS, foi adotado o algoritmo Newton-Raphson desenvolvido por Camargo [23].

\subsubsection{Obtenção do mecanismo de colapso}

O mecanismo de colapso é obtido plotando, na geometria do problema estudado, as velocidades obtidas do problema dual de otimização, no qual são estabelecidas com base no método dos multiplicadores de Lagrange. Assim como para o problema primal tem-se as tensões e o fator de colapso, no problema dual resultante as velocidades são as variáveis.

O campo de velocidades obtido permite identificar a superfície de ruptura, fazendo uma identificação qualitativa da mesma, já que o valor dessas velocidades não é representativo.

\subsubsection{Diagrama de fluxo geral da implementação}

Na figura 3.6, é apresentado um diagrama de fluxo geral, seguido na resolução dos problemas estudados, com ALN. 


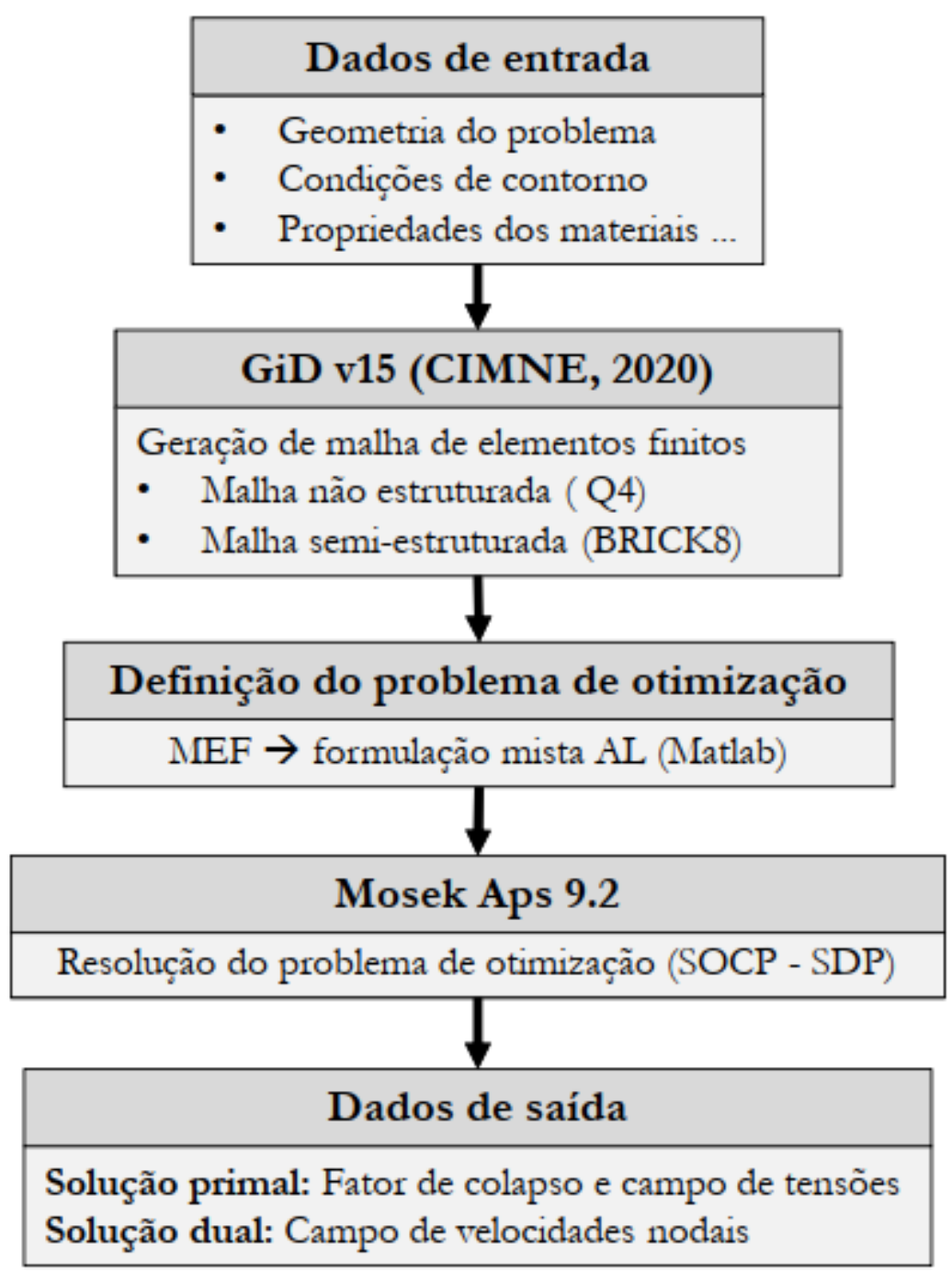

Figura 3.6: Diagrama de fluxo geral da implementação da ALN 


\section{4 \\ RESULTADOS}

O foco principal da pesquisa, para a aplicação dos códigos desenvolvidos, foi a análise de estabilidade de um poço, cuja geometria é apresentada na Figura 4.1. A análise foi subdividida nas seguintes fases, dependendo das condições do problema consideradas:

- Fase 1: Análise de estabilidade do poço (sem considerar anel de concreto e a presença da água)

- Fase 2: Análise de estabilidade do poço com anel de concreto

- Fase 3: Análise de estabilidade do poço com anel de concreto e considerando a presença do nível da água

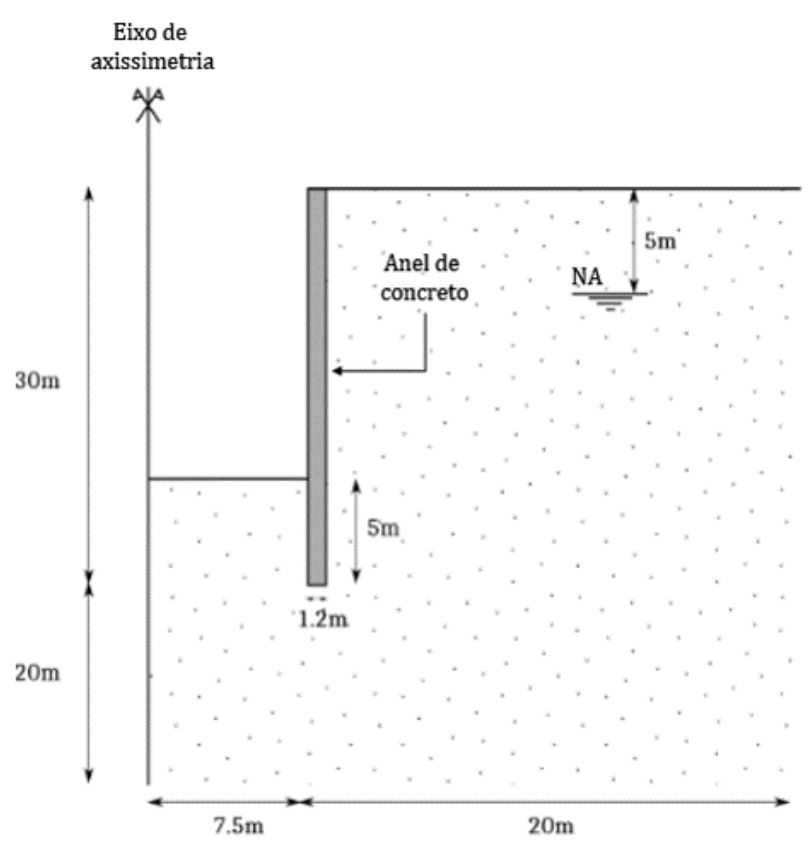

Figura 4.1: Geometria do poço estudado.

Além disso foi feita uma análise comparativa com resultados obtidos na literatura para o caso de estimação da capacidade de carga de uma fundação circular rasa. 
Para cada caso estudado, a forma geral da equação de equilíbrio do problema de otimização será reformulada, com a finalidade de esclarecer o tratamento dado a cada um deles. Nesse trabalho os códigos foram implementados no software MATLAB R2019b e o processador usado para todos os exemplos foi Intel Core i7-7700-CPU 3.60GHz, para a geração de malhas de elementos finitos e identificação do mecanismo de colapso foi utilizado o software de pré e pós processamento GiD v15 (CIMNE, 2020).

\subsection{Fase 1: Analise de estabilidade de poço}

\subsubsection{Considerações do caso estudado}

Inicialmente foi estudado o caso mais simples do problema do poço, sem considerar nem a presença do anel de concreto, nem o efeito das poropressões. A análise foi efetuada em várias etapas, que incluíram: a validação dos resultados obtidos do código no estado axissimétrico com os obtidos para o estado tridimensional, a execução de uma análise de sensibilidade ao refinamento da malha e por fim a comparação de resultados obtidos para diferentes parâmetros do material com as análises efetuadas nos softwares OPTUM G2 e PLAXIS 2D.

Neste caso a equação de equilíbrio do problema foi colocada como:

$$
[G]\{\sigma\}=\lambda\left\{f_{w}\right\}
$$

onde $\left\{f_{w}\right\}$ é o vetor de cargas nodais (força de volume) correspondente ao peso próprio da estrutura geotécnica.

\subsubsection{Validação Analise Axissimétrico - Analise Tridimensional}

Como uma forma de validar os resultados obtidos do código implementado para o estado axissimétrico, foram efetuadas análises para um modelo tridimensional do problema, com base no código desenvolvido por Garcia [27]. Para tal fim foi eleito o critério D-P com SOCP, fazendo uma aproximação aos parâmetros M-C por meio do Cone 2 (Tabela 2.1).

Na Figura 4.2 é apresentada a geometria e a malha semi-estruturada empregada na discretização do modelo tridimensional do poço. A profundidade do poço é de 25 metros e o raio de 7.5 metros. No modelo as condições de contorno foram impostas como é apresentado na Tabela 4.1.

Para a análise axissimétrica, foi gerada uma malha plana não estrutu- 
Tabela 4.1: Condições de contorno no modelo tridimensional do poço

\begin{tabular}{llll}
\hline \multirow{2}{*}{ Contorno } & \multicolumn{3}{l}{ Restrito em } \\
\cline { 2 - 4 } & x & y & Z \\
\hline 1 & & & \\
2 & $\checkmark$ & & \\
3 & & $\checkmark$ & \\
4,5 e 6 & $\checkmark$ & $\checkmark$ & $\checkmark$ \\
\hline
\end{tabular}

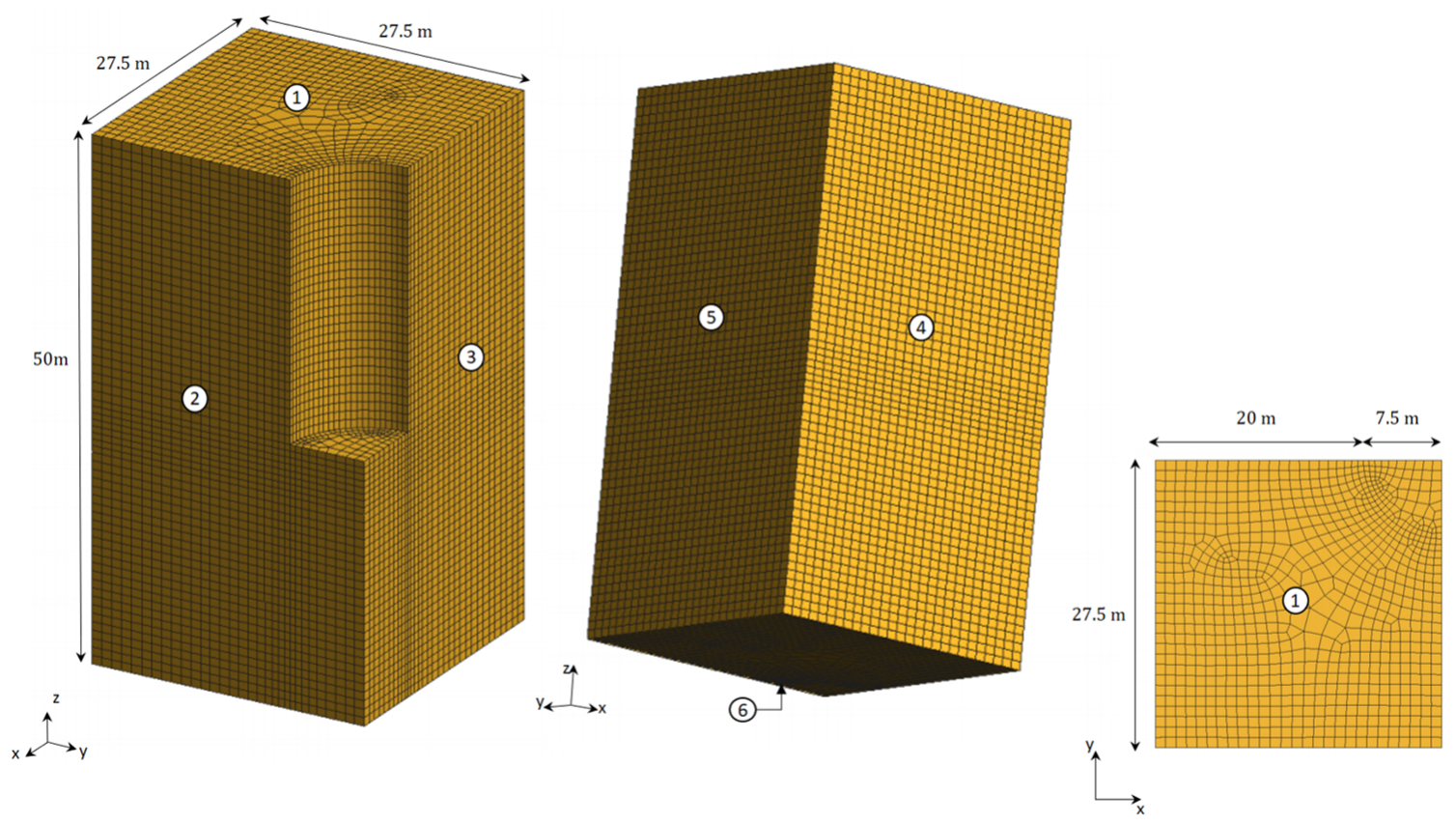

Figura 4.2: Geometria e malha semi-estruturada tridimensional do poço empregada na validação da análise axissimétrica.

rada, como é apresentado na Figura 4.3, onde também se pode conferir as dimensões do modelo. As condições de contorno foram impostas tal como é apresentado na Tabela 4.2.

Tabela 4.2: Condições de contorno no modelo axissimétrico do poço

\begin{tabular}{llc}
\hline \multirow{2}{*}{ Contorno } & \multicolumn{2}{r}{ Restrito em } \\
\cline { 2 - 3 } & r & Z \\
\hline 1 & $\checkmark$ & \\
2 & $\checkmark$ & $\checkmark$ \\
3 & $\checkmark$ & \\
4,5 e 6 & & \\
\hline
\end{tabular}

Os resultados das análises efetuadas para diferentes parâmetros do solo podem ser examinadas na Tabela 4.3. Nela se pode perceber a notável redução dos tempos de processamento requeridos no modelo axissimétrico 

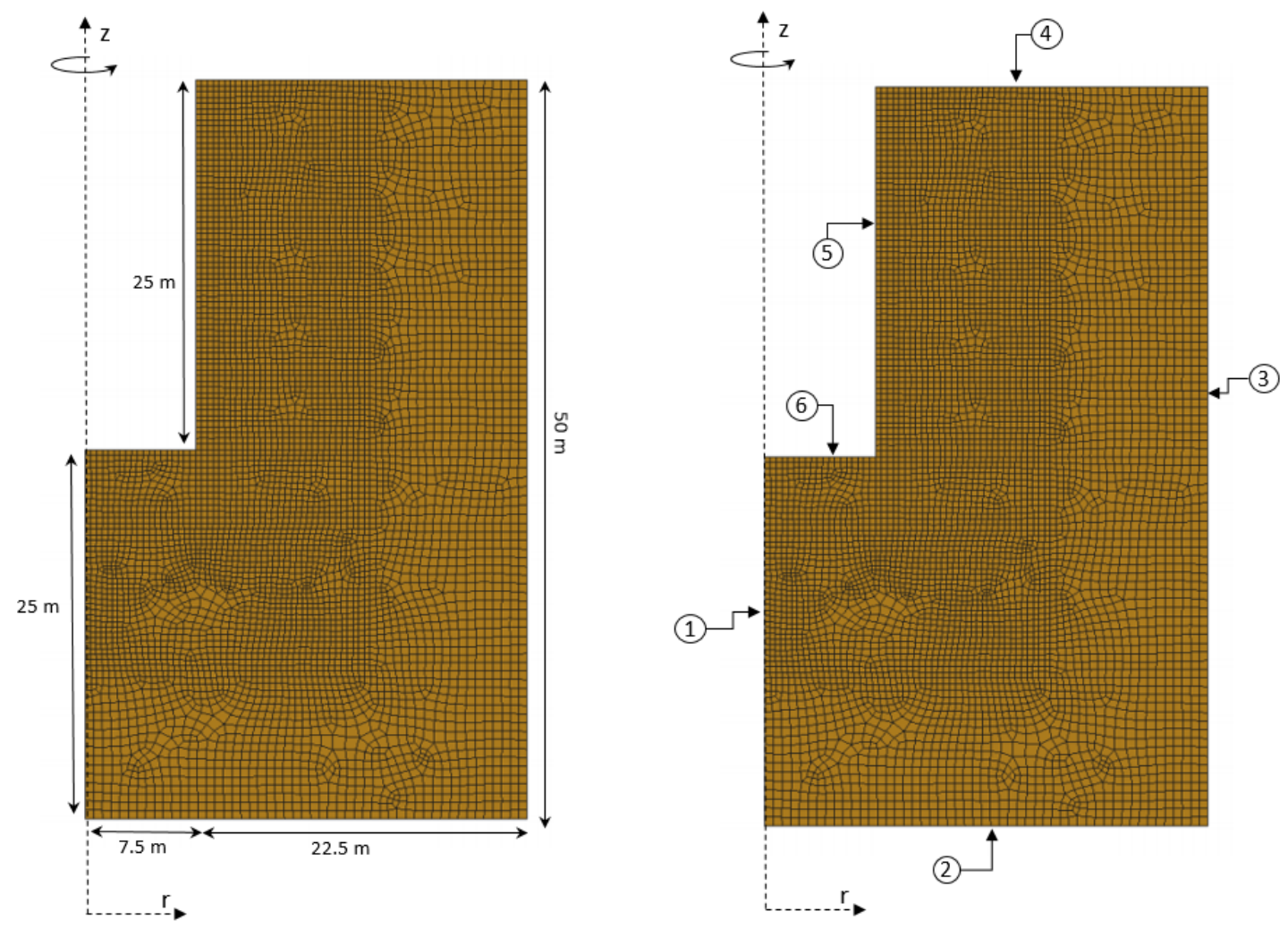

Figura 4.3: Geometria e malha não estruturada plana do poço empregada na validação da análise axissimétrica.

em comparação com o tridimensional e a diferença percentual na estimação do fator de segurança em todos os casos não supera o $1 \%$.

Tabela 4.3: Comparação entre os resultados obtidos nas análises axissimétrica e tridimensional (SOCP D-P cone 2)

\begin{tabular}{llcccc}
\hline \multirow{4}{*}{ Parâmetros } & Caso & 1 & 2 & 3 & 4 \\
\cline { 2 - 6 } & $\gamma\left(k N / m^{3}\right)$ & 18 & 16 & 17 & 17 \\
& $c(k P a)$ & 30 & 25 & 10 & 35 \\
& $\phi\left(^{\circ}\right)$ & 10 & 20 & 35 & 25 \\
\hline \multirow{2}{*}{ Número de elementos } & Analise Axissimétrico & 6278 & 6278 & 6278 & 6278 \\
\multirow{2}{*}{ Tempo de processamento $(\mathrm{s})$} & Analise 3D & 51081 & 51081 & 51081 & 51081 \\
& Analise Axissimétrico & 13.87 & 21.16 & 17.82 & 14.21 \\
FS & Analise 3D & 658.97 & 501.15 & 750.13 & 523.69 \\
Diferença percentual & Analise Axissimétrico & 0.75 & 0.93 & 0.80 & 1.22 \\
& Analise 3D & 0.76 & 0.94 & 0.80 & 1.22 \\
& & 0.45 & 0.49 & 0.86 & 0.49 \\
\hline
\end{tabular}

Os mecanismos de colapso obtidos pelo campo de velocidades nos modelos tridimensional e axissimétrico são muito parecidos, como podese corroborar nas Figuras 4.4 e 4.5, para os parâmetros do caso 3. De fato 
se é feita uma seção do modelo tridimensional, o mecanismo de colapso representado nela será idêntico ao conseguido no modelo axissimétrico.
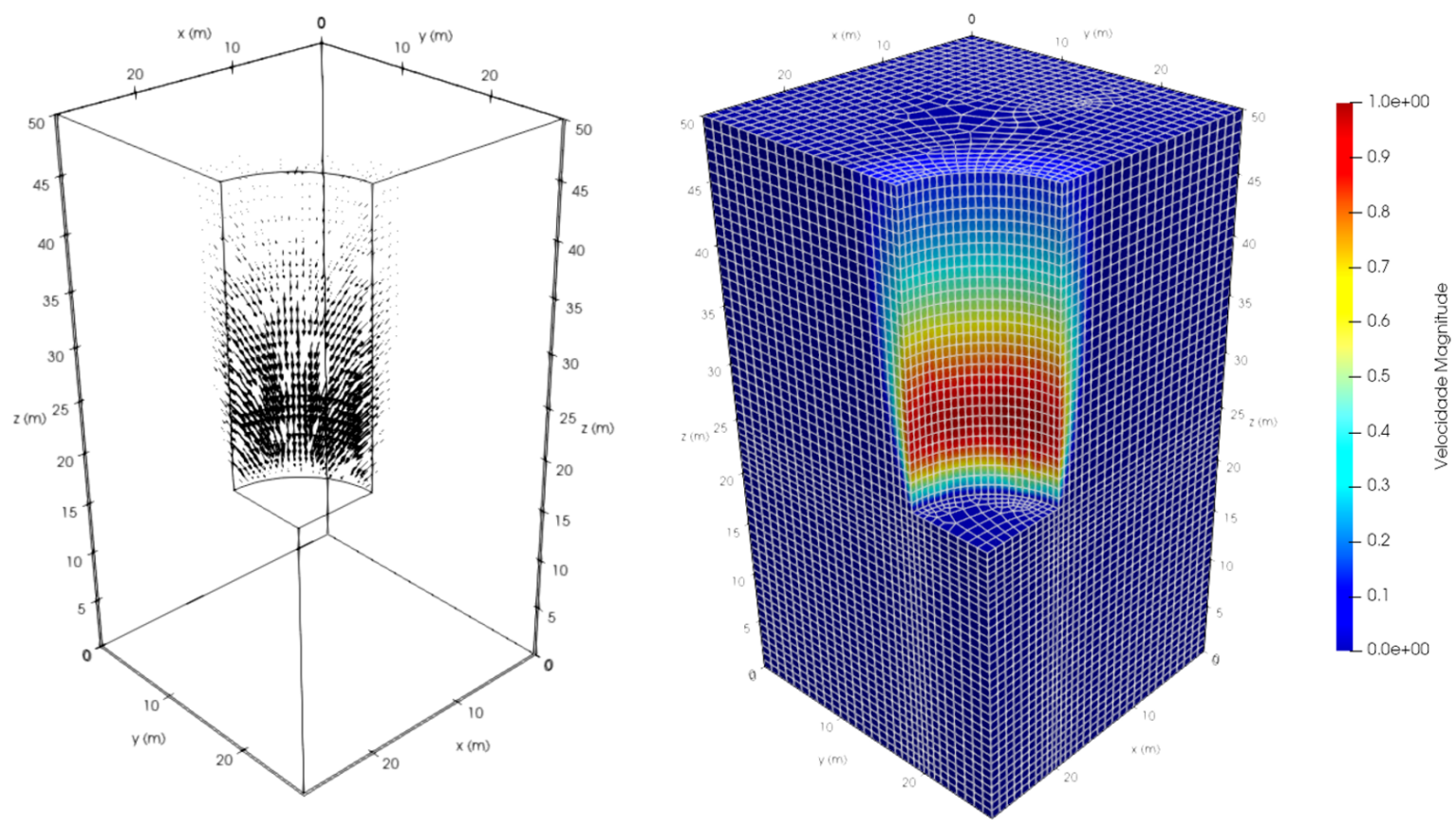

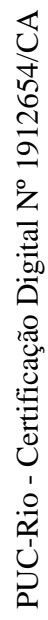

Figura 4.4: Mecanismo de colapso obtido na análise tridimensional (caso 3).
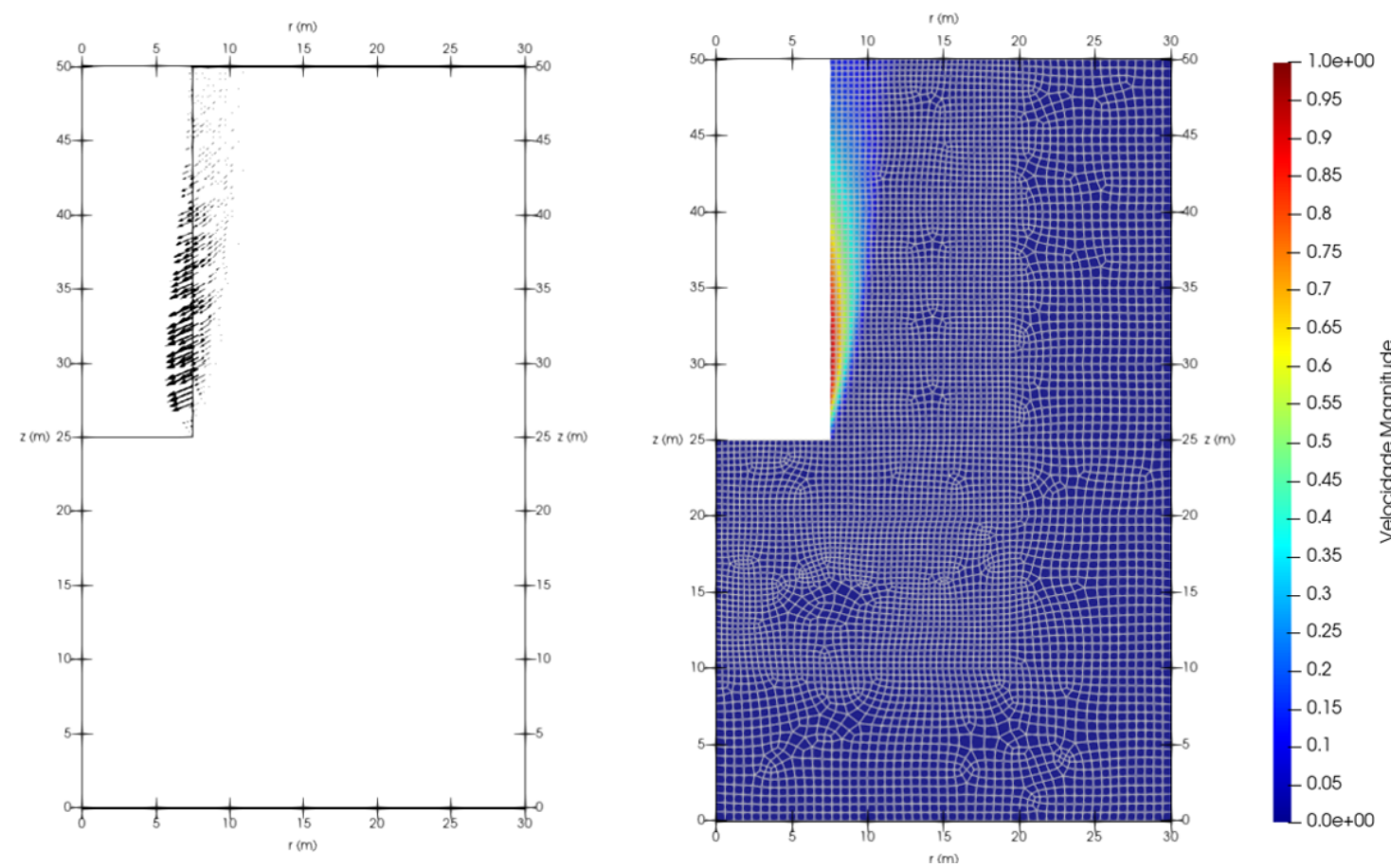

Figura 4.5: Mecanismo de colapso obtido na análise axissimétrica (caso 3). 


\subsubsection{Analise de refinamento da malha}

Uma vez validada a análise axissimétrica com a tridimensional, foram efetuadas análises de refinamento geral da malha, com a finalidade de verificar a variação do fator de segurança $F S$ com o acréscimo do número de elementos finitos. Para esse fim foram empregados tanto o critério D-P com SOCP, quanto o critério M-C como SDP.

Os parâmetros do solo considerados nas análises foram:

$$
\begin{aligned}
\gamma & =19 \mathrm{kN} / \mathrm{m}^{3} \\
c & =50 \mathrm{kPa} \\
\phi & =35^{\circ}
\end{aligned}
$$

No caso do critério D-P os parâmetros foram aproximados por meio dos Cones 1 e 2. Na Figura 4.6 são apresentados os resultados das análises, das quais se pode atentar o seguinte:

- Os gráficos para as 3 análises feitas começaram a exibir uma tendência assintótica antes dos 4000 elementos, para um número maior de elementos a variação do $F S$ resultou inferior a $0.3 \%$.

- Em geral, a sensibilidade da análise ao refinamento foi baixa, por exemplo, a diferença percentual do $F S$ obtido em malhas com 2154 elementos e 15655 resultou inferior ao $0.95 \%$ nas 3 análises.

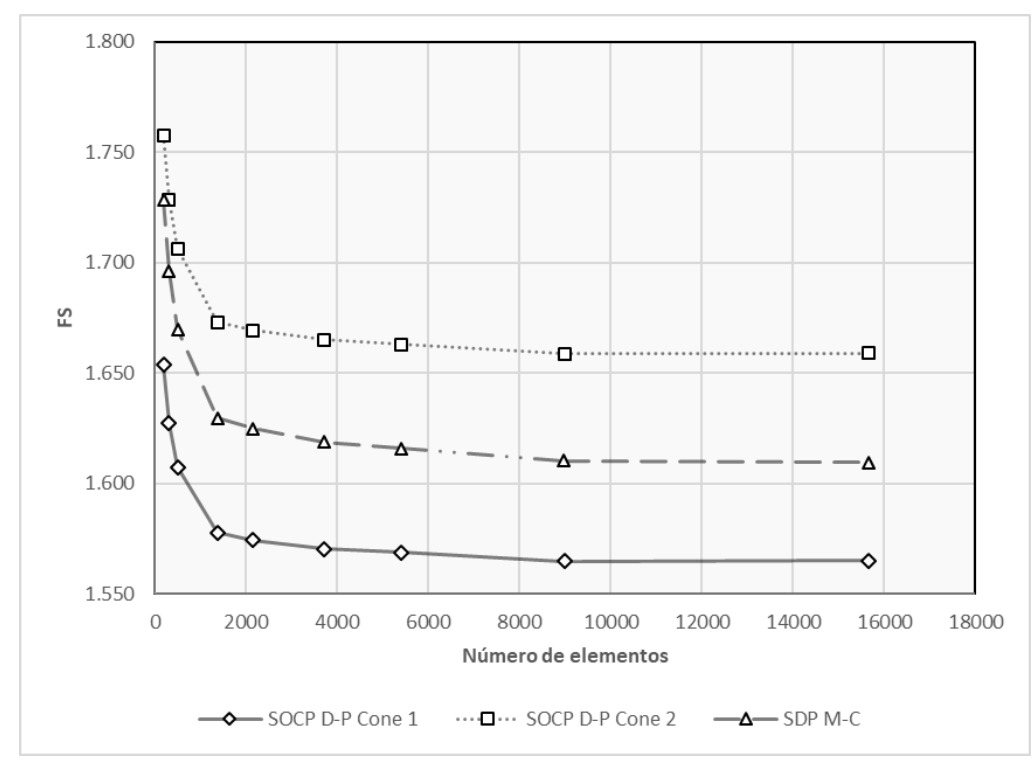

Figura 4.6: Analise de refinamento da malha. 
A respeito do mecanismo de colapso, na Figura 4.7 é possível perceber a melhoria sensível na definição da superfície de ruptura com o refinamento da malha.

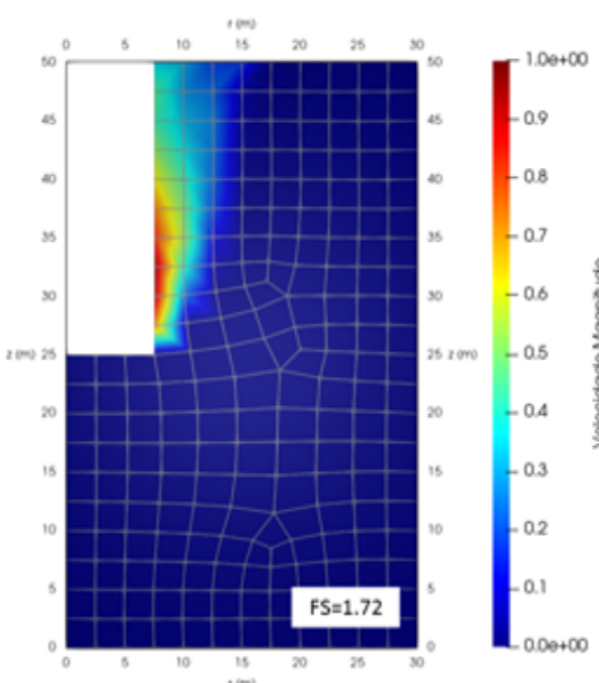

(a)

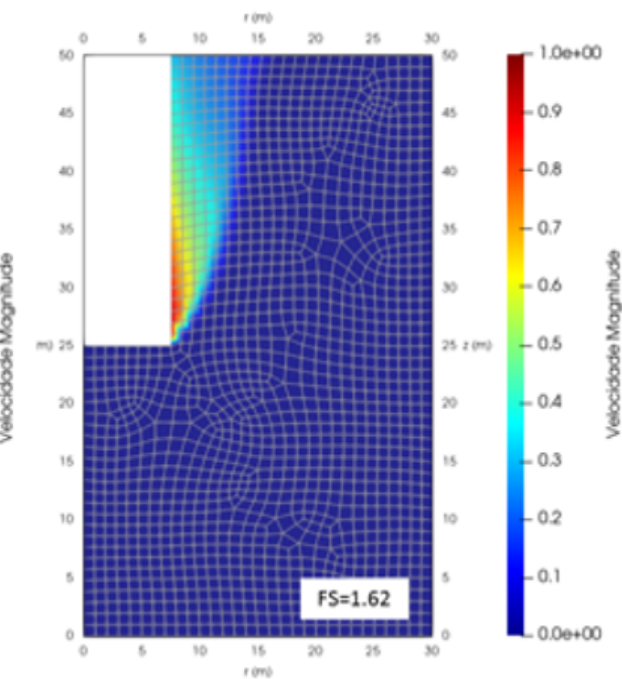

(b)

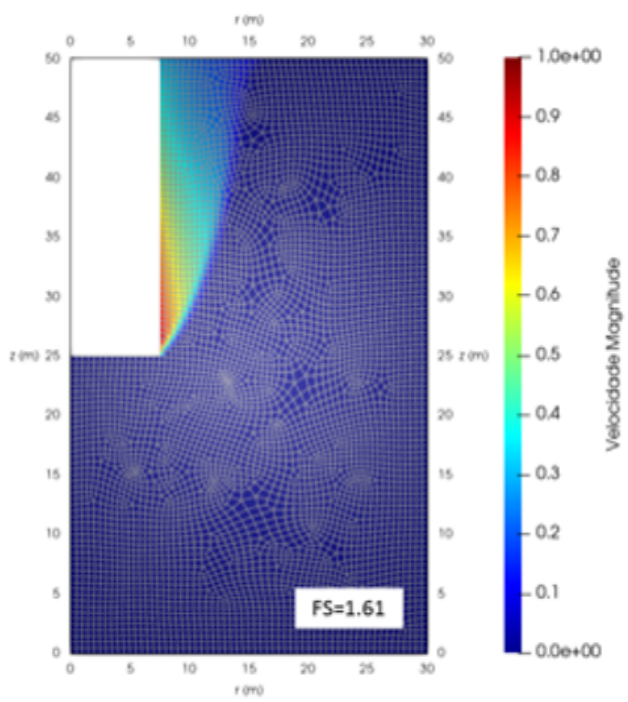

(c)

Figura 4.7: Mecanismos de colapso obtidos na análise de refinamento geral da malha, com programação SDP M-C(a) 198 elementos (b) 1371 elementos (c) 5404 elementos.

\subsubsection{Analise de estabilidade para diferentes parâmetros do solo}

Na Tabela 4.4 são apresentados os resultados das análises efetuadas na condição axissimétrica, para 8 casos de parâmetros do solo, com o objetivo de estudar principalmente os seguintes aspectos: 
- O desempenho na estimação do $F S$ com SOCP de todos os Cones D-P presentes na Tabela 2.1, tendo como referência os resultados obtidos do critério M-C formulado com SDP.

- A diferença dos resultados obtidos nos códigos de ALN implementados nesta pesquisa, com os obtidos do software de Analise Elastoplástica Plaxis 2D e o software de ALN Optum G2.

Nas análises foram criadas malhas não estruturadas de mais de 5000 elementos e para Plaxis 2D e Optum G2 foram empregadas malhas de 2293 e 2000 elementos respectivamente ${ }^{1}$.

Tabela 4.4: Fator de Segurança obtido para diferentes casos de parâmetros do solo na análise de estabilidade

\begin{tabular}{clcccccccc}
\hline \multirow{5}{*}{ Parâmetros } & Caso & 1 & 2 & 3 & 4 & 5 & 6 & 7 & 8 \\
\cline { 2 - 9 } & $\gamma\left(k N / m^{3}\right)$ & 18 & 16 & 17 & 18 & 18 & 18 & 16 & 19 \\
& $c(k P a)$ & 5 & 10 & 15 & 25 & 25 & 30 & 40 & 50 \\
& $\phi\left(^{\circ}\right)$ & 35 & 30 & 25 & 15 & 30 & 20 & 0.1 & 35 \\
\hline \multirow{5}{*}{ FS } & SOCP D-P Cone 1 & 0.58 & 0.71 & 0.74 & 0.72 & 1.02 & 0.91 & 0.65 & 1.56 \\
& SOCP D-P Cone 2 & 0.59 & 0.73 & 0.77 & 0.77 & 1.07 & 0.97 & 0.75 & 1.66 \\
& SOCP D-P Cone 3 & 0.85 & 0.90 & 0.89 & 0.82 & 1.21 & 1.04 & 0.75 & 1.80 \\
& SOCP D-P Cone 4 & 0.91 & 0.97 & 0.95 & 0.86 & 1.29 & 1.10 & 0.75 & 1.91 \\
& SOCP D-P Cone 5 & 1.19 & 1.18 & 1.12 & 0.96 & 1.50 & 1.23 & 0.75 & 2.16 \\
& SDP M-C & 0.59 & 0.73 & 0.76 & 0.74 & 1.05 & 0.94 & 0.69 & 1.61 \\
& M-C Optum G2 & 0.58 & 0.72 & 0.75 & 0.74 & 1.05 & 0.93 & 0.69 & 1.61 \\
& M-C Plaxis & 0.51 & 0.65 & 0.69 & 0.71 & 0.98 & 0.89 & 0.66 & 1.53 \\
\hline
\end{tabular}

No que diz respeito ao desempenho dos Cones D-P com SOCP, por meio da Figura 4.8 é possível evidenciar que as melhores aproximações ao critério M-C foram feitas pelos Cones 1 e 2, enquanto que os Cones 3, 4 e 5 resultaram em uma superestimação do $F S$.

Para o Cone 1 a diferença percentual média na estimação do $F S$ com respeito aos resultados obtidos do critério M-C com SDP, foi de 3.01\% (inferior), e no Cone 2 foi de $2.85 \%$ (superior).

Na Figura 4.9 foram representados os $F S$ obtidos do critério D-P com SOCP (Cone 1 e 2), do critério M-C com SDP e dos softwares Optum G2 e Plaxis 2D, para os 8 casos, os valores determinados neste trabalho foram muito próximos aos estimados com o software de ALN Optum G2, de fato

\footnotetext{
${ }^{1}$ Os resultados apresentados para Optum G2, resultam do valor medio obtido das estimações rigorosas pelo límite inferior e superior, na análise foi efetuado o processo de adaptação da malha disponível neste software.
} 


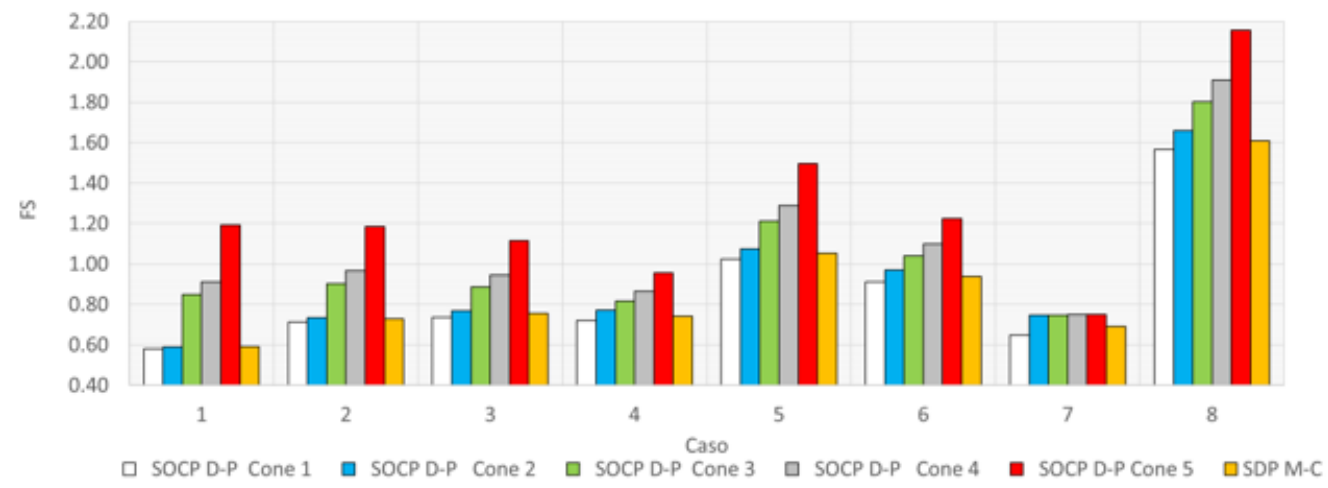

Figura 4.8: Comparação entre os resultados obtidos com os cones D-P (SOCP) e M-C (SDP).

para a análise SDP M-C a diferença percentual media foi de $0.61 \%$ e de $2.42 \%$ e $3.47 \%$ para a análise SOCP D-P com o Cone 1 e 2 respectivamente.

Com respeito aos $F S$ conseguidos do Plaxis 2D, eles resultaram inferiores em todos os casos aos obtidos da ALN e as diferenças percentuais medias foram maiores que as estimadas com Optum G2, sendo de 8.24\% para a análise SDP M-C e de 5.01\% e 11.26\% para SOCP D-P com o Cone 1 e 2 respectivamente.

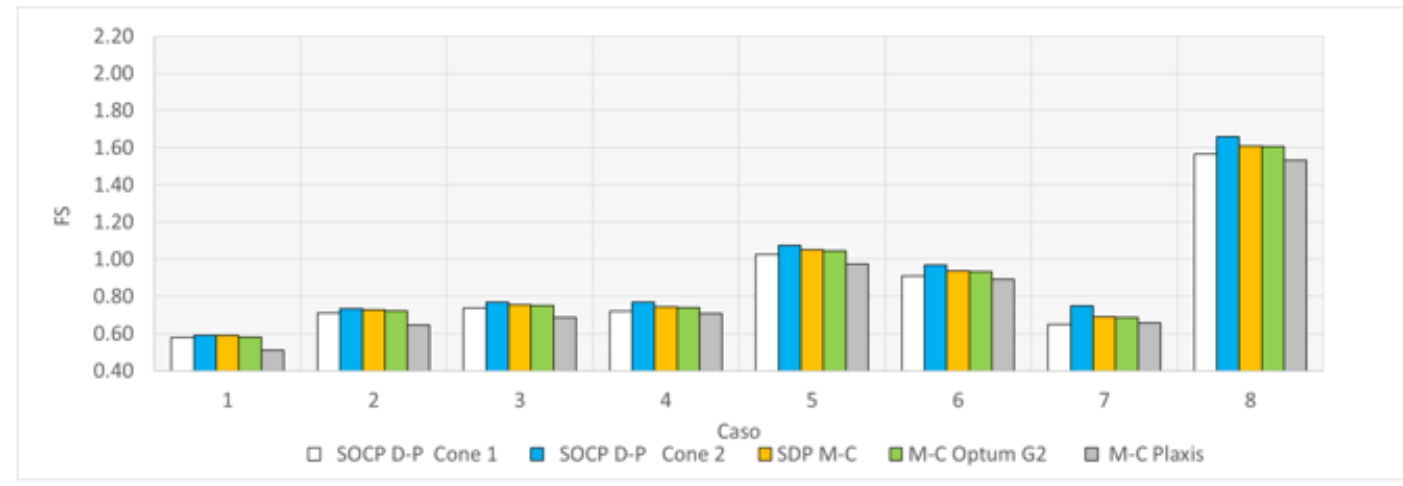

Figura 4.9: Comparação dos resultados conseguidos neste trabalho com os resultados obtidos em Optum G2 e Plaxis 2D.

O tempo médio de processamento foi inferior a 10 segundos nas análises com SOCP, de 190 segundos nas analises com SDP, de 41 segundos no Plaxis 2D e de 34 segundos no Optum G2. Dos 8 casos de parâmetros estudados, apenas nos casos 5 e 8 foram obtidos $F S$ maiores de 1, apresentando 
o colapso da estrutura geotécnica para essas condições. Na Figura 4.10 são apresentados os mecanismos de colapso identificados no caso 7 para os métodos de análise empregados, como se pode perceber a representação da superfície de ruptura é idêntica.

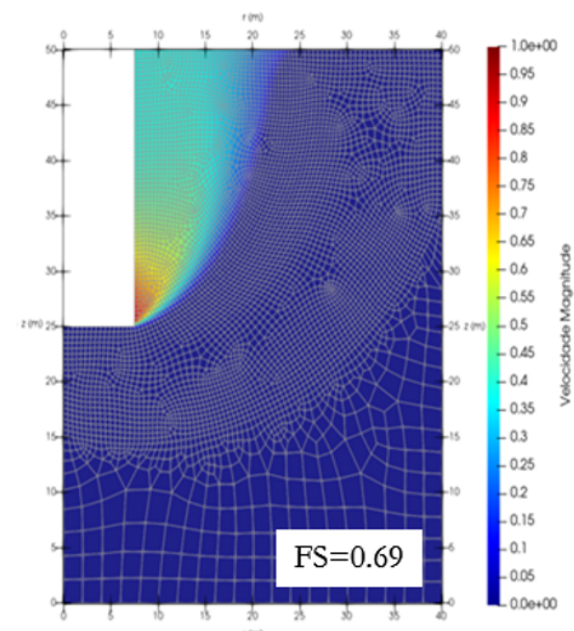

(a)

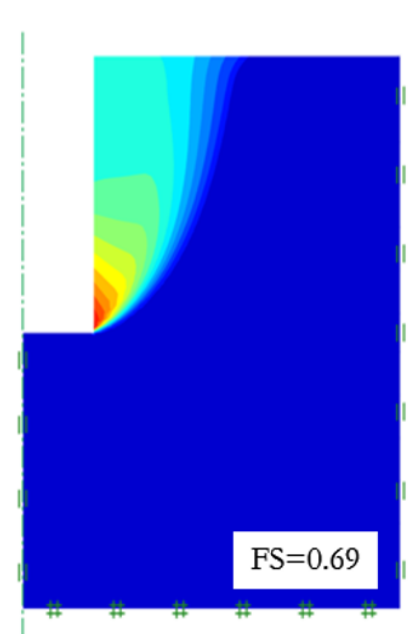

(b)

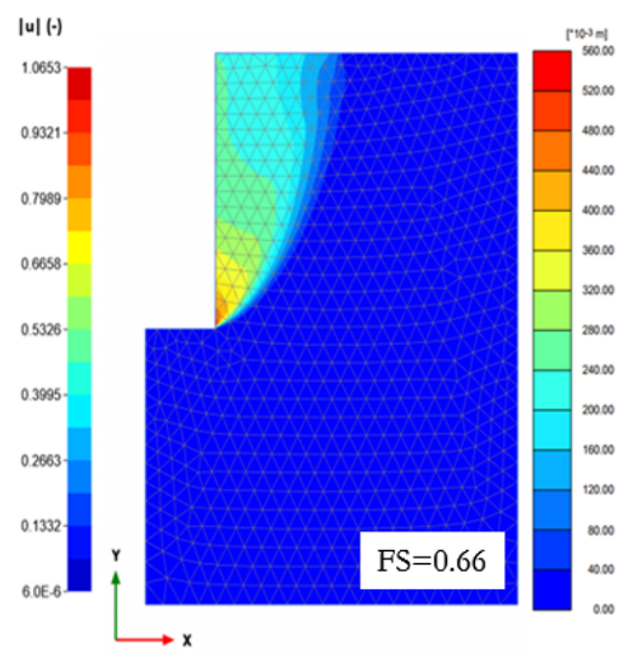

(c)

Figura 4.10: Mecanismo de colapso para o caso 7. (a) Análise limite axissimétrica, programação SDP-MC (b) Optum G2 (c) Plaxis 2D.

\subsubsection{Analise de estabilidade de poço considerando heterogeneidade}

Com a finalidade de considerar o efeito da heterogeneidade na estabilidade do poço, foram abordados 2 casos nos quais tem-se um contraste nos parâmetros das camadas de solo pertencentes à estrutura geotécnica. Foram realizadas análises SOCP D-P com o Cone 1 e 2 e SDP M-C, com condições de contorno iguais às da Tabela 4.2 e mantendo as dimensões do poço da Figura 4.3. Também foram feitas as análises para verificação de resultados nos softwares Optum G2 e Plaxis 2D.

\subsubsection{Caso 1: Camada mais resistente na base}

O primeiro caso de heterogeneidade estudado no poço, considera um perfil estratigráfico conformado por duas camadas, sendo a camada 2, na base, a mais resistente. Na Figura 4.11 é apresentada a geometria do problema e a malha não estruturada utilizada na análise.

Os resultados obtidos na análise de estabilidade, em termos do $F S$ são apresentados na Tabela 4.5, com base na qual se podem fazer as seguintes 
observações:

- O FS obtido em todas as análises foi inferior a 1, o que implica o estado de colapso da estrutura para essas condições

- As estimações do FS feitas pelas diferentes metodologias apresentaram resultados muito próximos aos obtidos do Optum G2, principalmente as feitas com SOCP D-P Cone 1 e SDP M-C.

- Seguindo a tendência dos exemplos anteriormente estudados, a programação SOCP apresento tempos de processamento bastante inferiores aos requeridos com a programação SDP e o Plaxis 2D.
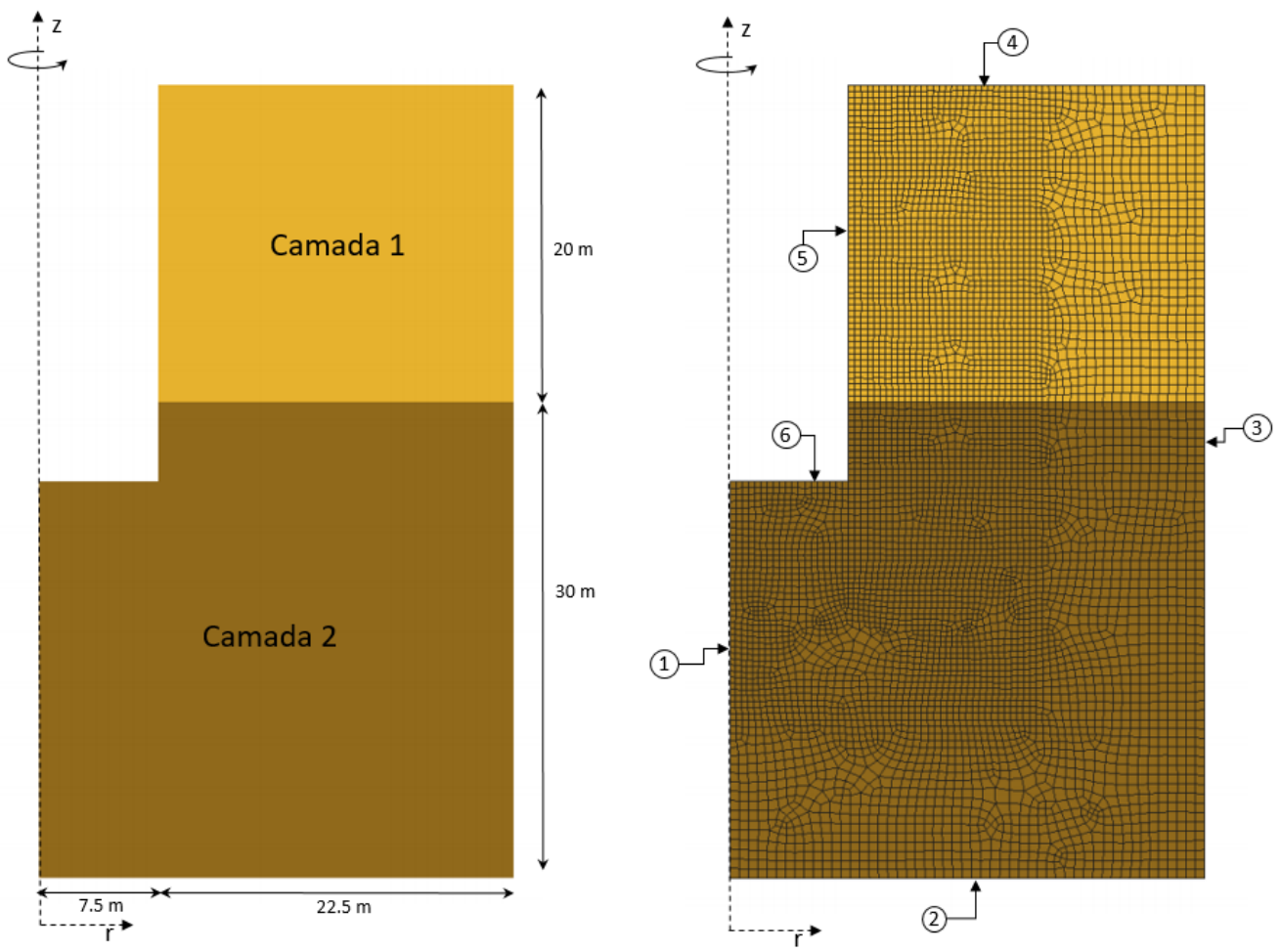

Figura 4.11: Geometria e malha não estruturada do caso 1 de heterogeneidade.

Um dos aspectos mais interessantes na inclusão de heterogeneidade no modelo é sua influência na definição da superfície de ruptura, da Figura 4.12 se pode verificar que para o caso estudado a posição da camada resistente resulta fundamental na definição do mecanismo de colapso. 
Tabela 4.5: Resultados caso 1 de heterogeneidade, com camada mais resistente na base

\begin{tabular}{llll}
\hline \multirow{4}{*}{ Parâmetros } & Camada & 1 & 2 \\
\cline { 2 - 4 } & $\gamma\left(k N / m^{3}\right)$ & 17 & 18 \\
& $c(k P a)$ & 20 & 40 \\
& $\phi\left(^{\circ}\right)$ & 10 & 35 \\
\hline
\end{tabular}

\begin{tabular}{lccccc}
\hline \multirow{2}{*}{ Tipo de análise } & FS & Tempo de processamento (s) & \multirow{2}{*}{ Número de nós } & \multicolumn{2}{c}{ Diferença percentual } \\
\cline { 5 - 6 } & & & & OPTUM G2 & PLAXIS \\
\hline SOCP D-P Cone 1 & 0.60 & 8.19 & 6443 & 2.87 & 0.28 \\
SOCP D-P Cone 2 & 0.65 & 7.86 & 6443 & 5.11 & 8.51 \\
SDP M-C & 0.62 & 190.68 & 6443 & 0.43 & 3.68 \\
M-C Optum G2 & 0.62 & 13.31 & 6003 & & \\
MC Plaxis & 0.60 & 62.00 & 7675 & & \\
\hline
\end{tabular}

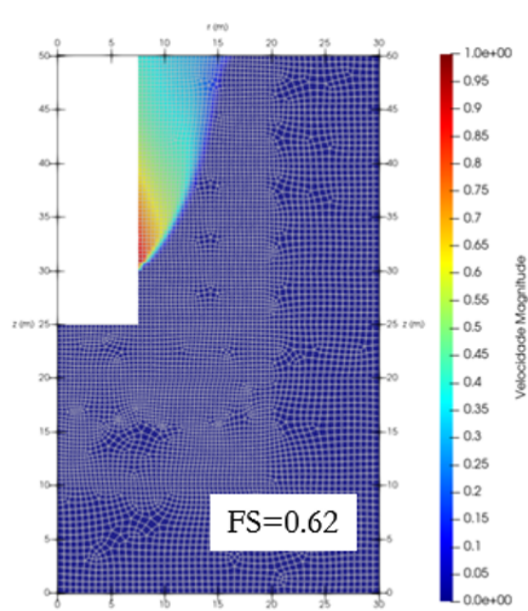

(a)

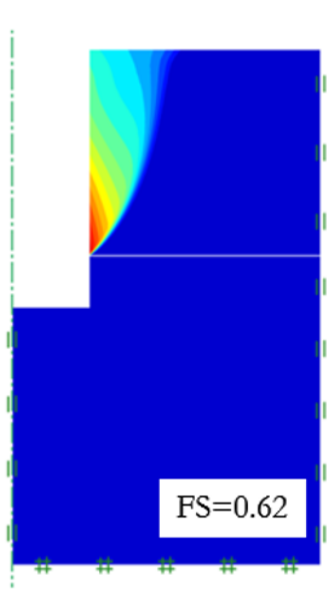

(b)

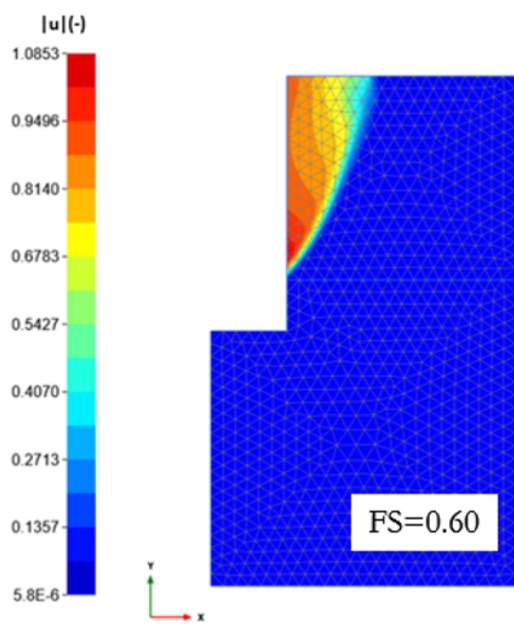

(c)

Figura 4.12: Mecanismo de colapso, caso 1 de heterogeneidade. (a) Análise limite programação SDP M-C (b) Optum G2 (c) Plaxis 2D.

\subsubsection{Caso 2: Presença de camada menos resistente}

O caso 2 de heterogeneidade contempla a presença de uma camada menos resistente de 5 metros de espessura na estratigrafia do poço (Figura 4.13). Da Tabela 4.6 com os resultados da análise se pode inferir que os $F S$ estimados pelas diferentes metodologias foram inferiores a 1, evidenciando o estado de colapso para as condições dadas. A maior diferença percentual foi obtida entre a análise feita com SOCP D-P Cone 2 e o Plaxis 2D, correspondente a um $7.79 \%$. Como nos exemplos anteriores a programação SOCP demandou os menores tempos de processamento e a programação SDP junto com o Plaxis 2D os maiores.

Na Figura 4.14 foi representado o mecanismo de colapso obtido pelas 


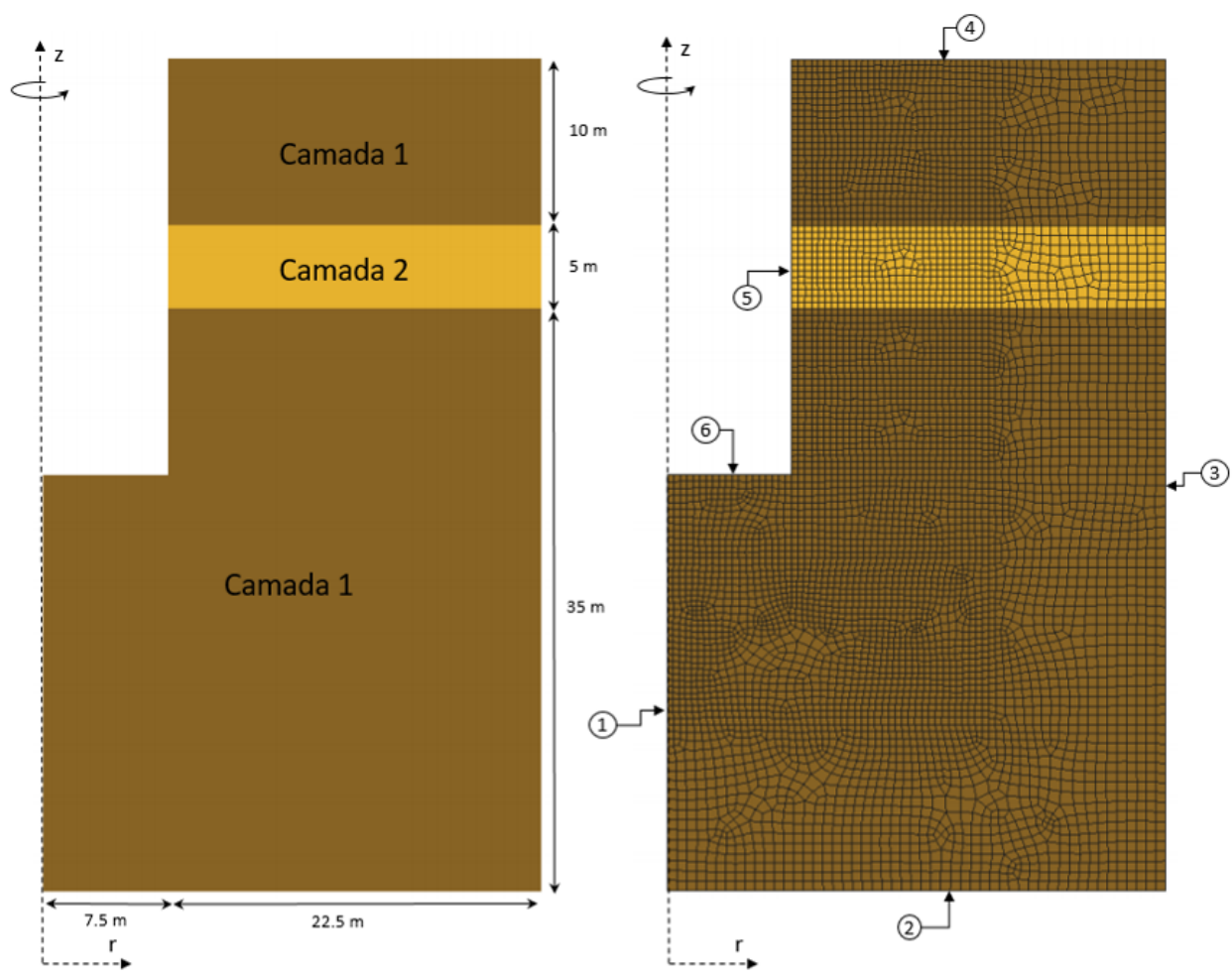

Figura 4.13: Geometria e malha não estruturada do caso 2 de heterogeneidade.

Tabela 4.6: Fator de segurança estimado no caso 2 de heterogeneidade, com presença de camada menos resistente

\begin{tabular}{|c|c|c|c|c|c|}
\hline \multirow{4}{*}{ Parâmetros } & & \multicolumn{2}{|l|}{ Camada } & \multirow{2}{*}{$\begin{array}{c}1 \\
18\end{array}$} & \multirow{2}{*}{$\begin{array}{c}2 \\
18\end{array}$} \\
\hline & & $\gamma\left(k N / m^{3}\right)$ & & & \\
\hline & & $c(k P a)$ & & 10 & 5 \\
\hline & & $\phi\left(^{\circ}\right)$ & & 35 & 15 \\
\hline \multirow[t]{2}{*}{ Tipo de análise } & \multirow[t]{2}{*}{ FS } & \multirow[t]{2}{*}{ Tempo de processamento (s) } & \multirow[t]{2}{*}{ Número de nós } & $\begin{array}{l}\text { Diferença } \\
\text { tual }\end{array}$ & percen- \\
\hline & & & & OPTUM G2 & PLAXIS \\
\hline SOCP D-P Cone 1 & 0.48 & 8.65 & 6443 & 5.05 & 0.07 \\
\hline SOCP D-P Cone 2 & 0.49 & 8.47 & 6443 & 1.38 & 3.80 \\
\hline SDP M-C & 0.51 & 172.97 & 6443 & 2.41 & 7.79 \\
\hline M-C Optum G2 & 0.50 & 16.86 & 6000 & & \\
\hline MC Plaxis & 0.48 & 106.00 & 7295 & & \\
\hline
\end{tabular}

diferentes metodologias, a superfície de ruptura foi similar em todas as análises e a presença e posição da camada de solo menos resistente foi fundamental em sua definição.

\subsubsection{Comparação com resultados presentes na literatura, em termos do número de estabilidade}

Para finalizar a fase 1 da análise, foi feita uma comparação com os resultados apresentados por Sun e Yang [16] e outros autores, para o caso 


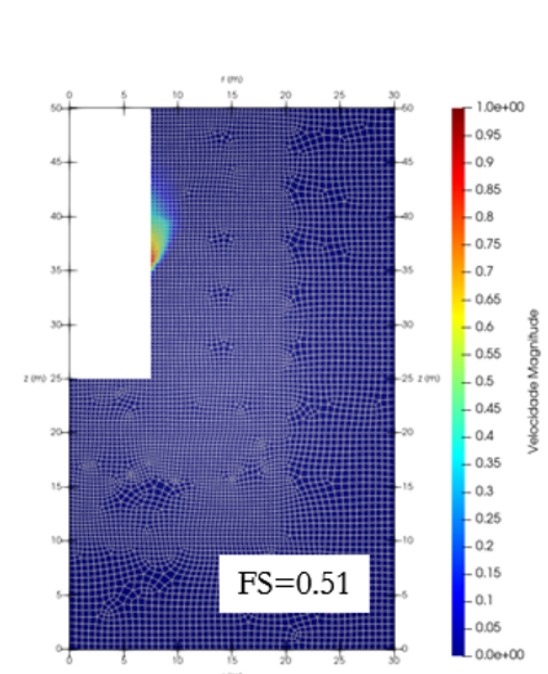

(a)

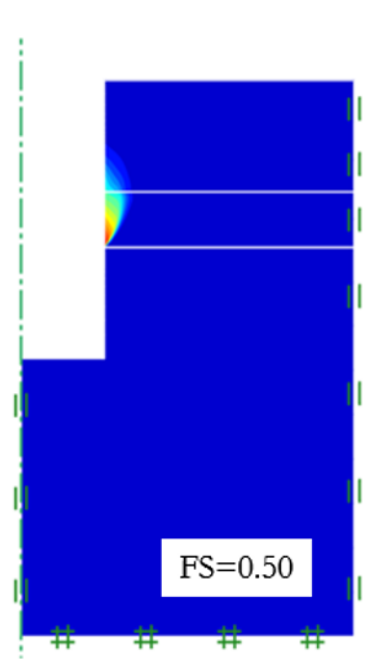

(b)

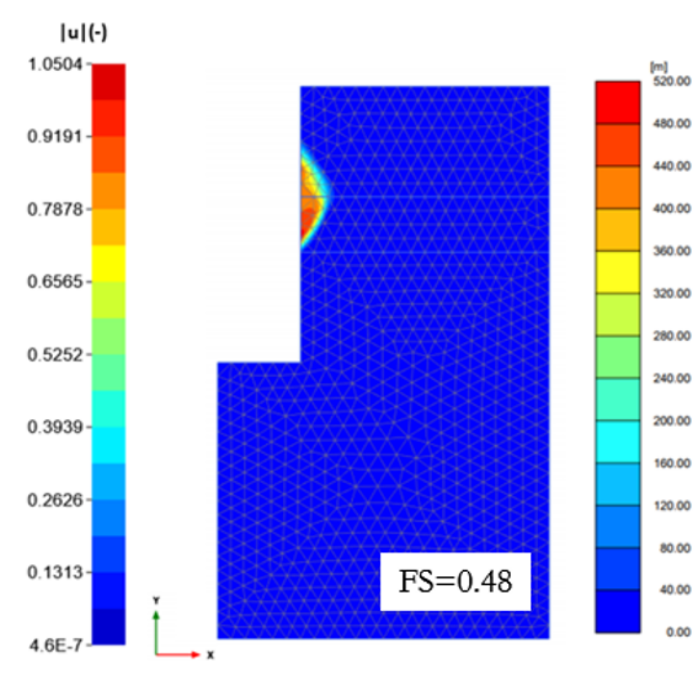

(c)

Figura 4.14: Mecanismo de colapso, caso 2 de heterogeneidade. (a) Análise limite programação SDP M-C (b) Optum G2 (c) Plaxis 2D.

de dois poços, cada um com diferentes relações H/R (Profundidade/Raio). As geometrias e as malhas não estruturadas com refinamento local na zona de interesse, são apresentadas na Figura 4.15.

Nas análises efetuadas para esses casos, a estabilidade foi avaliada em termos do número de estabilidade $N_{s}$, definido como:

$$
N_{s}=\frac{\gamma_{\max } H}{c}
$$

onde $\mathrm{H}$ é a profundidade do poço, c é a coesão (considerada de $1 \mathrm{kPa}$ para as análises) e $\gamma_{\max }$ é o peso específico máximo do solo do poço. $\mathrm{O} \gamma_{\max }$ é obtido da otimização do fator de colapso $\lambda$ que multiplica a $\left\{f_{w}\right\}$ da equação (4-1), neste caso, resultante da integração de $\{F\}=\{0,-1\}^{T}$ onde o $-1^{2}$ corresponde ao peso específico do solo em $k N / m^{3}$ predefinido para facilitar os cálculos.

Da Tabela (4.7) que apresenta os resultados das análises feitas nesta pesquisa e os resultados de outros autores, nos quais se implementaram formulações rigorosas pelos limites inferior (LI) ou superior (LS), podem-se fazer as seguintes observações:

- A diferença percentual máxima dos $N_{s}$ obtidos nas análises executados nesta pesquisa e os obtidos por Sun e Yang foi de $4.56 \%, 9.32 \%$ e

${ }^{2}$ Sinal negativo devido à orientação da força 

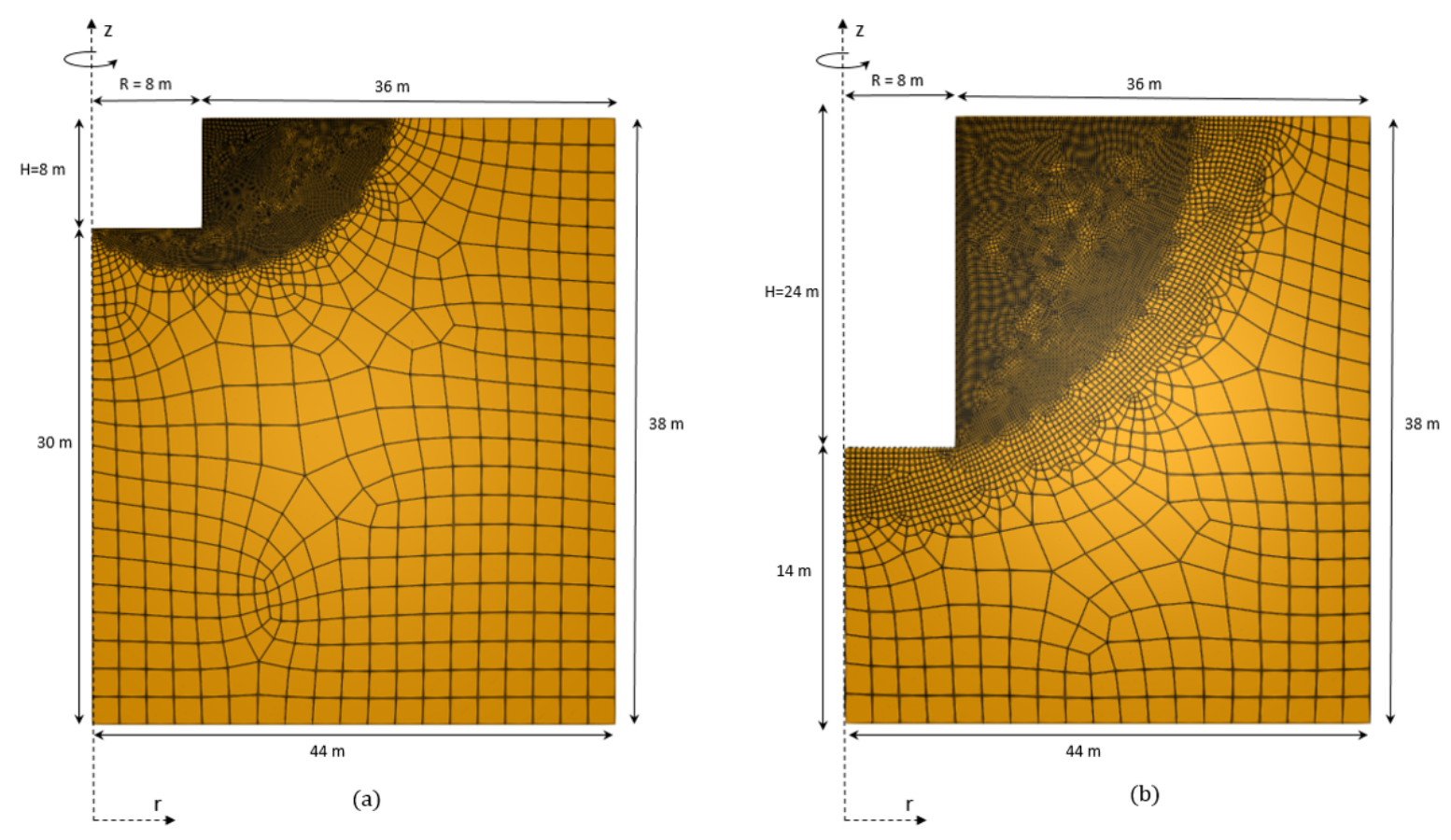

Figura 4.15: Geometrias e malhas não estruturadas dos poços (a) relação

$H / R=1$, malha de 9270 elementos (b) relação $H / R=3$, malha de 9814 elementos.

1.01\% para as programações SOCP D-P Cone 1, Cone 2 e SDP M-C respectivamente.

- A estimação do $N_{s}$ feita com SOCP D-P Cone 1 resultou ser a mais conservadora das análises comparadas, entanto que os $N_{s}$ obtidos com o Cone 2 resultaram em alguns casos ligeiramente maiores que os obtidos com formulações do limite superior (Kumar et al, Lyamin e Sloan e Zhang et al).

- Os $N_{s}$ resultantes da análise SDP M-C na maioria dos casos ficaram entre os valores dos limites superior e inferior das análises de Kumar et al. e Lyamin e Sloan.

Os mecanismos de colapso identificados neste trabalho foram semelhantes aos obtidos nas análises de Sun e Yang, nas quais a superfície de ruptura é definida mediante um processo de refinamento adaptativo da malha, como se pode conferir nas Figuras 4.16 e 4.17. 
Tabela 4.7: Comparação do número de estabilidade $\left(N_{s}\right)$ obtido para diferentes valores de ângulo de atrito $(\phi)$

\begin{tabular}{|c|c|c|c|c|c|c|c|c|c|c|}
\hline \multirow[t]{2}{*}{$\mathbf{H} / \mathbf{R}$} & \multirow[t]{2}{*}{$\phi\left(\left(^{\circ}\right)\right.$} & \multicolumn{3}{|c|}{$\begin{array}{l}\text { Resultados do presente tra- } \\
\text { balho }\end{array}$} & \multirow{2}{*}{$\frac{\begin{array}{l}\text { Sun e Yang } \\
(2020)\end{array}}{\text { LI }}$} & \multicolumn{2}{|c|}{$\begin{array}{l}\text { Kumar et al. } \\
(2014) \dagger\end{array}$} & \multirow{2}{*}{$\frac{\begin{array}{c}\text { Lyamin } \\
(2002)^{*}\end{array}}{\text { LS }}$} & \multirow{2}{*}{$\begin{array}{l}\text { e Sloan } \\
\text { LI }\end{array}$} & \multirow{2}{*}{$\begin{array}{l}\text { Zhang et al. } \\
(2018) \dagger \\
\text { LS }\end{array}$} \\
\hline & & $\begin{array}{l}\text { SOCP D-P } \\
\text { cone } 1\end{array}$ & $\begin{array}{l}\text { SOCP D-P } \\
\text { cone } 2\end{array}$ & SDP M-C & & LS & LI & & & \\
\hline \multirow{5}{*}{1} & 0 & - & - & 5.15 & 5.12 & 5.17 & 4.93 & 5.26 & 5.09 & 5.11 \\
\hline & 10 & 6.26 & 7.06 & 6.50 & 6.46 & 6.49 & 6.20 & 6.73 & 6.48 & 6.41 \\
\hline & 20 & 8.05 & 8.88 & 8.33 & 8.28 & 8.37 & 7.98 & 8.85 & 8.30 & 8.11 \\
\hline & 30 & 10.60 & 11.43 & 10.97 & 10.89 & 10.82 & 10.41 & - & - & 10.53 \\
\hline & 40 & 14.51 & 15.34 & 15.12 & 14.97 & 15.59 & 14.25 & - & - & 14.45 \\
\hline \multirow{5}{*}{3} & 0 & - & - & 6.65 & 6.62 & 6.75 & 6.45 & 6.83 & 6.59 & - \\
\hline & 10 & 8.62 & 9.81 & 9.07 & 9.03 & 9.01 & 8.80 & 9.42 & 9.00 & - \\
\hline & 20 & 12.10 & 13.50 & 12.70 & 12.64 & 12.78 & 12.24 & 13.43 & 12.54 & - \\
\hline & 30 & 17.61 & 19.22 & 18.47 & 18.38 & 17.83 & 17.72 & - & - & - \\
\hline & 40 & 27.13 & 28.97 & 28.50 & 28.23 & 29.62 & 27.03 & - & - & \\
\hline
\end{tabular}

$\left(^{*}\right)$ Analise feita com modelo tridimensional

$(\dagger)$ Na formulação é aplicada a hipótese de Harr-von Karman (tratamento especial dado a $\sigma_{\theta \theta}$ como tensão principal)

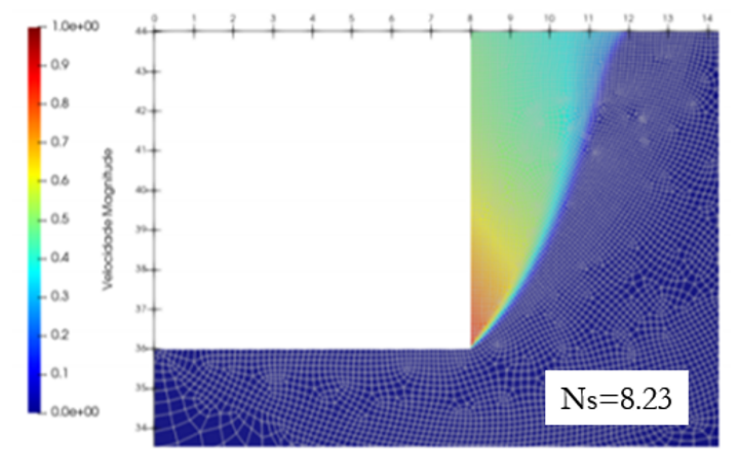

(a)

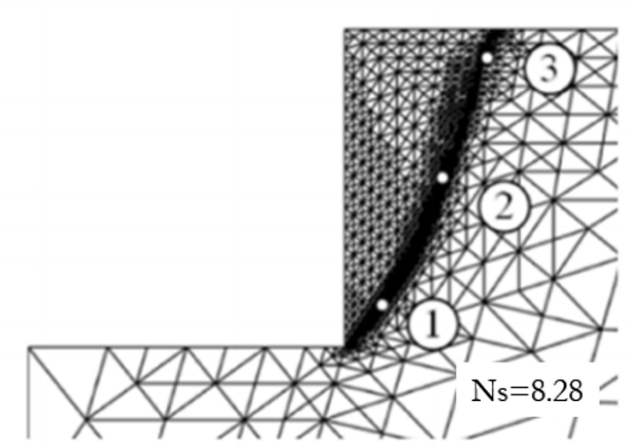

(b)

Figura 4.16: Mecanismo de colapso estimado para $\phi=20^{\circ}$ e $H / R=1$ por: (a) O presente trabalho SDP M-C (b) Sun e Yang (2020).

\subsection{Fase 2: Analise de estabilidade de poço com anel de concreto}

A fase 2 da análise do poço, contemplou a presença de um anel de concreto, elemento que contribui à estabilidade do poço, conforme visto na fase anterior, dada a geometria do problema se precisavam parâmetros do solo mais elevados para conseguir $F S$ próximos ou ligeiramente superiores a 1, portanto resulta fundamental a presença de um elemento estrutural que garanta a obtenção de $F S$ maiores.

\subsubsection{Condições do problema estudado}

À análise do poço foi incorporada a presença de um anel de concreto, com uma espessura da parede de 1.2 metros e um comprimento de 30 metros. A inclusão do anel na análise trouxe desafios na formulação do 


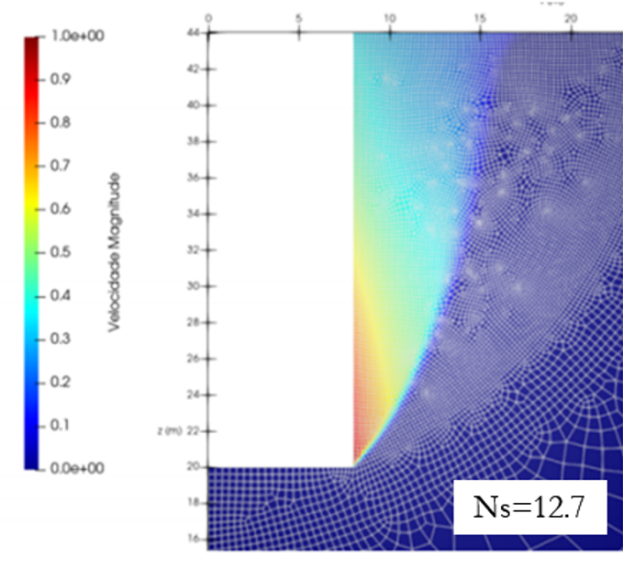

(a)

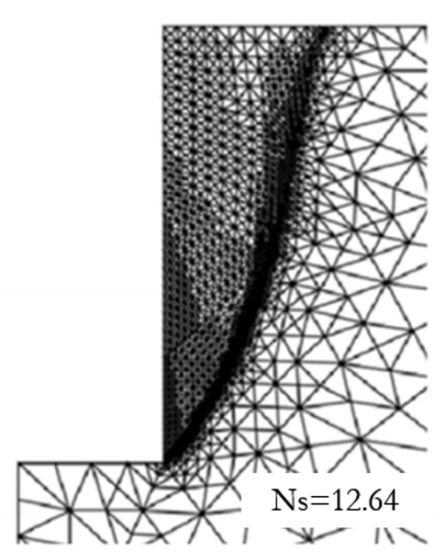

(b)

Figura 4.17: Mecanismo de colapso estimado para $\phi=20^{\circ}$ e $H / R=3$ por: (a) O presente trabalho SDP M-C (b) Sun e Yang (2020).

problema que foram abordados baseados nas seguintes considerações:

- O interesse da análise foi estudar a estabilidade do poço diante da ruptura do solo, sendo assim foi assumido que o anel de concreto não ia romper.

- Para os elementos pertencentes ao anel de concreto foram atribuídos parâmetros M-C suficientemente elevados para garantir o seu comportamento como material rígido. Para isto se fizeram análises de calibração do modelo.

- A redução dos parâmetros de resistência, requerida no processo de cálculo do $F S$, somente foi efetuada aos elementos finitos de solo, não aos pertencentes ao anel de concreto.

- Nesta fase foi requerida uma modificação da equação de equilíbrio do problema de otimização, que é apresentada em seguida.

\subsubsection{Modificação da equação de equilíbrio}

Para as análises da fase 2 a equação de equilíbrio sofre a seguinte modificação:

$$
[G]\{\sigma\}=\left\{f_{w}\right\}+\lambda\left\{f_{f}\right\}
$$

onde $\left\{f_{f}\right\}$ é um vetor de força de volume fictícia, que resulta da integração de $\left\{F_{f i c}\right\}=\{-1,0\}$, no caso axissimétrico e $\left\{F_{f i c}\right\}=\{0,-1,0\}$ no caso tridimensional, o sinal negativo indica a direção contraria ao eixo $r$ e $y$ 
respectivamente, como foi demonstrado em Camargo [23] a inclusão dessa força deixa o problema mais estável. $\mathrm{E}\left\{f_{w}\right\}$ é o vetor com o peso da estrutura geotécnica.

\subsubsection{Calibração dos parâmetros $M-C$ do anel de concreto}

Se fizeram análises no estado axissimétrico para os 6 casos de parâmetros do solo apresentados na Tabela 4.8, e considerando para o concreto um ângulo de atrito $\phi=60^{\circ}$, peso específico $\gamma=24 \mathrm{kN} / \mathrm{m}^{3}$ e coesão $(c)$ variável. A ideia foi realizar análises para cada tipo de solo, com os parâmetros do concreto $\gamma$ e $\phi$ definidos acima e para diferentes valores de coesão, com a finalidade de determinar a coesão do concreto necessária a fim de garantir o comportamento rígido do anel.

Tabela 4.8: Parâmetros de solo utilizados na análise de calibração

\begin{tabular}{cccccccc}
\hline & Caso & 1 & 2 & 3 & 4 & 5 & 6 \\
\cline { 2 - 7 } Parâmetros do solo & $\phi\left({ }^{\circ}\right)$ & 5 & 15 & 20 & 30 & 35 & 35 \\
& $c(k P a)$ & 30 & 10 & 8 & 5 & 25 & 50 \\
& $\gamma\left(k N / m^{3}\right)$ & 17 & 18 & 18 & 17 & 18 & 19 \\
\hline
\end{tabular}

Na Figura 4.18 são apresentados os resultados obtidos, dos quais é possível verificar o comportamento assintótico no cálculo de $F S$ que começam a apresentar as análises desde valores de coesão superiores a $1000 \mathrm{kPa}$. Esse comportamento assintótico reflete uma característica importante do análise, representa o ponto a partir do qual o mecanismo de colapso estudado pertence à ruptura do solo e não do anel de concreto. Para as análises apresentadas em seguida se adotou um valor de coesão para os elementos de concreto de $2000 \mathrm{kPa}$.

\subsubsection{Validação Analise Axissimétrico - Analise Tridimensional}

Do mesmo modo como foi realizada uma validação da análise axissimétrica com a tridimensional na fase 1, foi executada na fase 2, nas Figuras 4.19 e 4.20 são apresentadas as geometrias e as malhas utilizadas nas análises. No caso tridimensional o modelo foi discretizado por meio de uma malha semi-estruturada de 31590 elementos e no modelo tridimensional através de uma malha plana não estruturada de 6278 elementos. As condições de contorno impostas para o modelo tridimensional são apresentadas na Tabela 4.9 , e para a análise axissimétrica na Tabela 4.10. As análises foram feitas com SOCP D-P, Cone 2. 


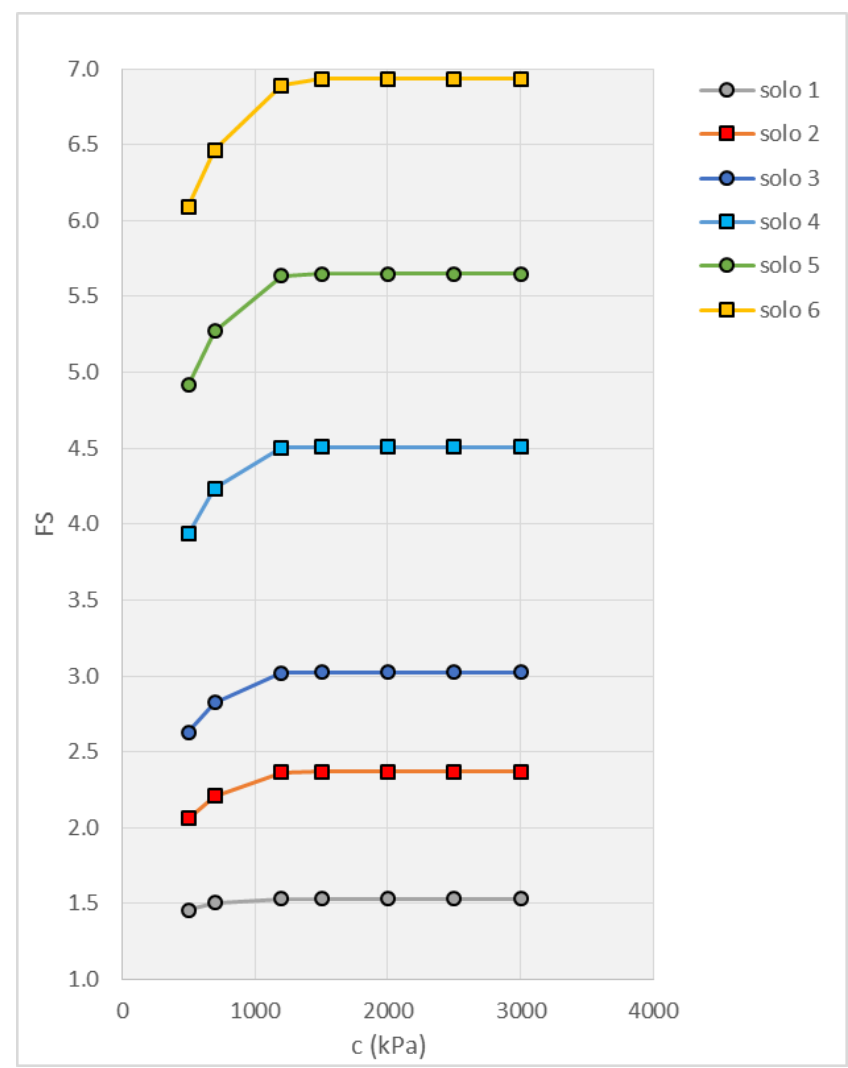

Figura 4.18: Resultados da análise de calibração dos parâmetros M-C do concreto.

Tabela 4.9: Condições de contorno no modelo tridimensional do poço com anel de concreto

\begin{tabular}{llll}
\hline \multirow{2}{*}{ Contorno } & \multicolumn{3}{l}{ Restrito em } \\
\cline { 2 - 4 } & x & y & Z \\
\hline 1 & & & \\
2 & $\checkmark$ & & \\
3 & & $\checkmark$ & \\
4,5 e 6 & $\checkmark$ & $\checkmark$ & $\checkmark$ \\
\hline
\end{tabular}

Tabela 4.10: Condições de contorno no modelo axissimétrico do poço com anel de concreto

\begin{tabular}{llc}
\hline \multirow{2}{*}{ Contorno } & \multicolumn{2}{c}{ Restrito em } \\
\cline { 2 - 3 } & r & z \\
\hline 1 & $\checkmark$ & \\
2 & $\checkmark$ & $\checkmark$ \\
3 & $\checkmark$ & \\
4,5 e 6 & & \\
\hline
\end{tabular}

Os resultados da validação são apresentados na Tabela 4.11, da qual podem fazer-se as seguintes observações

- A diferença percentual das análises tridimensional e axissimétrica é 

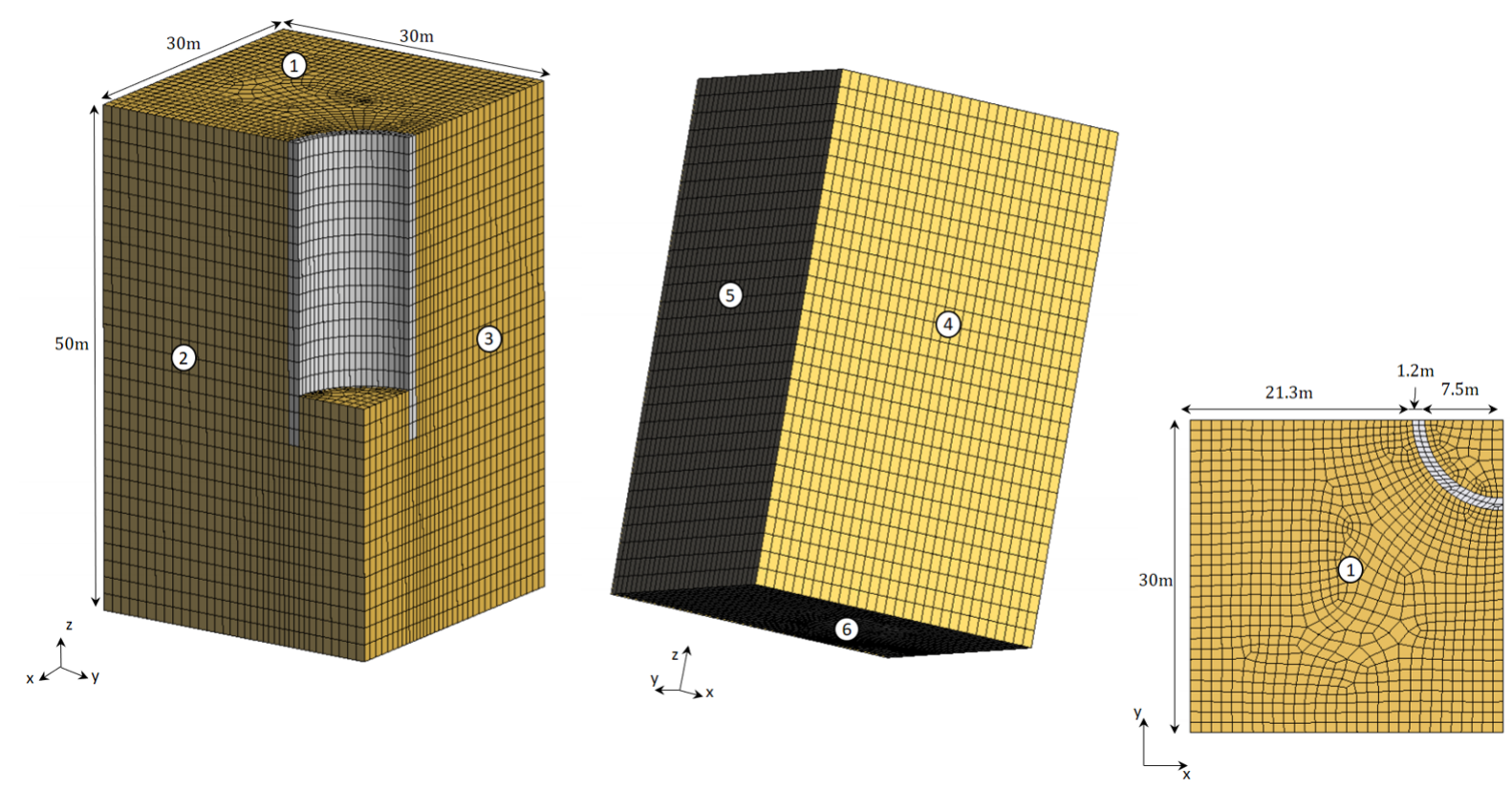

Figura 4.19: Geometria e malha semi-estruturada tridimensional do poço com anel de concreto, empregada na validação da análise axissimétrica.

Tabela 4.11: Comparação do Fator de Segurança obtido na análise axissimétrica e tridimensional, estabilidade do poço com anel de concreto

\begin{tabular}{llcccc}
\hline \multirow{4}{*}{ Parâmetros } & Caso & 1 & 2 & 3 & 4 \\
\cline { 2 - 6 } & $\gamma\left(\mathrm{kN} / \mathrm{m}^{3}\right)$ & 16 & 17 & 18 & 19 \\
& $c(k P a)$ & 6 & 10 & 10 & 50 \\
& $\phi\left(^{\circ}\right)$ & 20 & 25 & 20 & 35 \\
\hline \multirow{2}{*}{ Número de elementos } & Analise Axissimétrico & 6278 & 6278 & 6278 & 6278 \\
\multirow{2}{*}{ Tempo de processamento $(\mathrm{s})$} & Analise 3D & 31590 & 31590 & 31590 & 31590 \\
& Analise Axissimétrico & 15.22 & 13.44 & 16.71 & 22.94 \\
FS & Analise 3D & 439.82 & 427.93 & 428.54 & 305.54 \\
Diferença percentual & Analise Axissimétrico & 2.94 & 3.86 & 3.10 & 6.93 \\
& Analise 3D & 3.00 & 3.93 & 3.15 & 7.04 \\
& & 1.85 & 1.80 & 1.74 & 1.57 \\
\hline
\end{tabular}

baixa, inferior ao $2 \%$ em todos os casos, o que sugere que se faz uma boa representação do problema de estabilidade com o modelo axissimétrico.

- A diferença entre as análises, respeito ao tempo de processamento é considerável, as análises axissimétricas foram feitas em tempos inferiores a 23 segundos.

Os mecanismos de colapso identificados na analise tridimensional e axissimétrica são representados nas Figuras 4.21 e 4.22 respectivamente, 

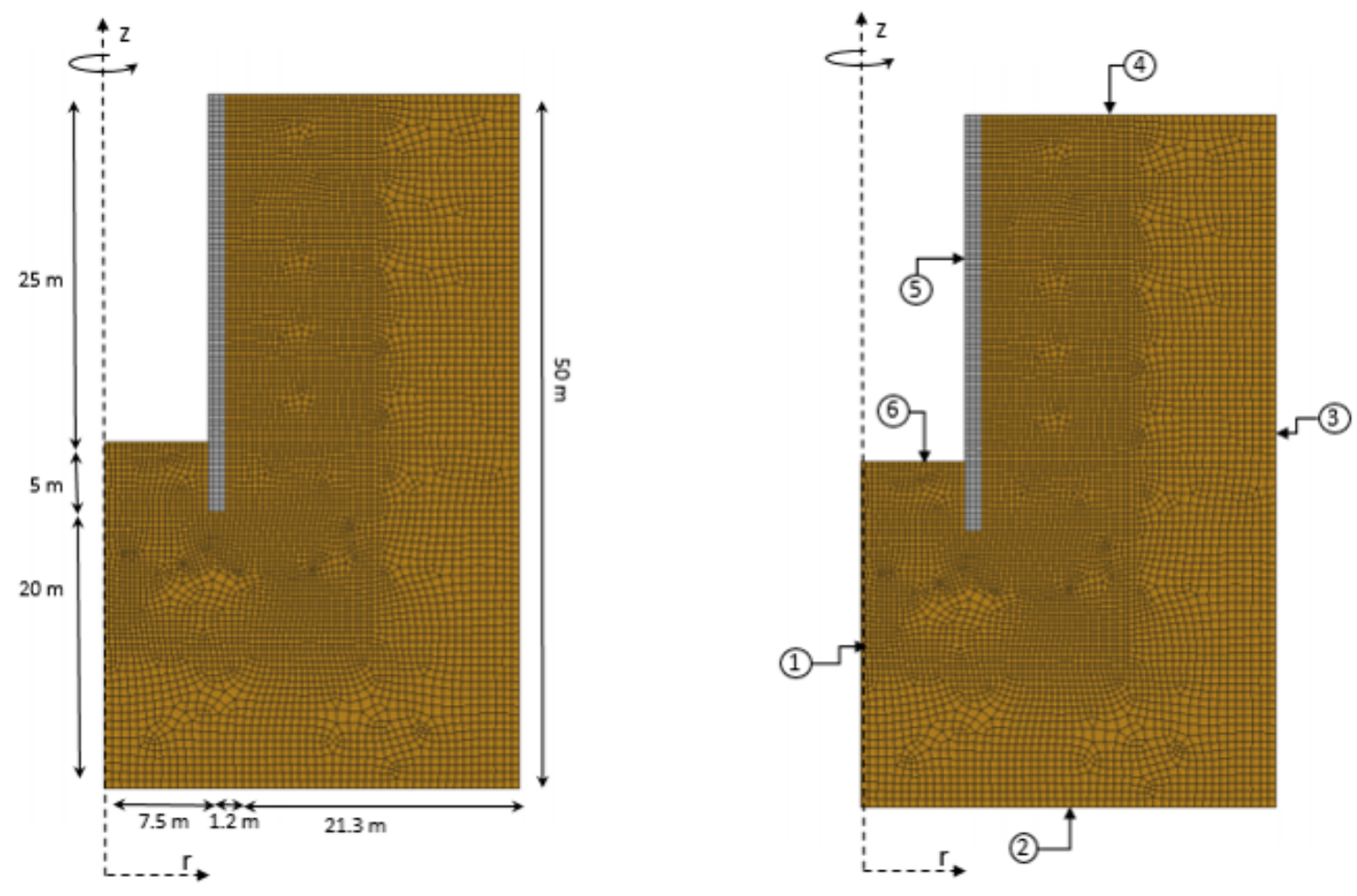

Figura 4.20: Geometria e malha não estruturada plana do poço com anel de concreto, empregada na validação da análise axissimétrica.

como se pode perceber são idênticos e representam a ruptura pela base do poço.

\subsubsection{Analise de estabilidade para diferentes parâmetros $M-C$ do solo}

Para diferentes casos de parâmetros $\mathrm{M}-\mathrm{C}$ do solo, foram efetuadas analises com programação SOCP D-P Cone 1 e 2 e com SDP M-C, com o fim de verificar os resultados também se fizeram utilizaram os softwares Plaxis 2D e Optum G2. No caso do Plaxis 2D o comportamento do material do anel de concreto foi considerado elástico, com um modulo de elasticidade $E=20 G P a$, um coeficiente de Poisson $v=0.2$ e um peso específico $\gamma=24 \mathrm{kN} / \mathrm{m}^{3}$, no caso de Optum G2 o material foi caracterizado como rígido, sendo somente necessário definir o peso específico do material $\gamma=$ $24 \mathrm{kN} / \mathrm{m}^{3}$.

Dos resultados, apresentados na Tabela 4.12 e representados na Figura 4.23, se podem fazer os seguintes comentários:

- A diferença percentual media com respeito aos $F S$ obtidos de Optum G2 foram de $4.03 \%, 7.33 \%$ e $1.59 \%$ para as analises SOCP D-P Cone 1, 

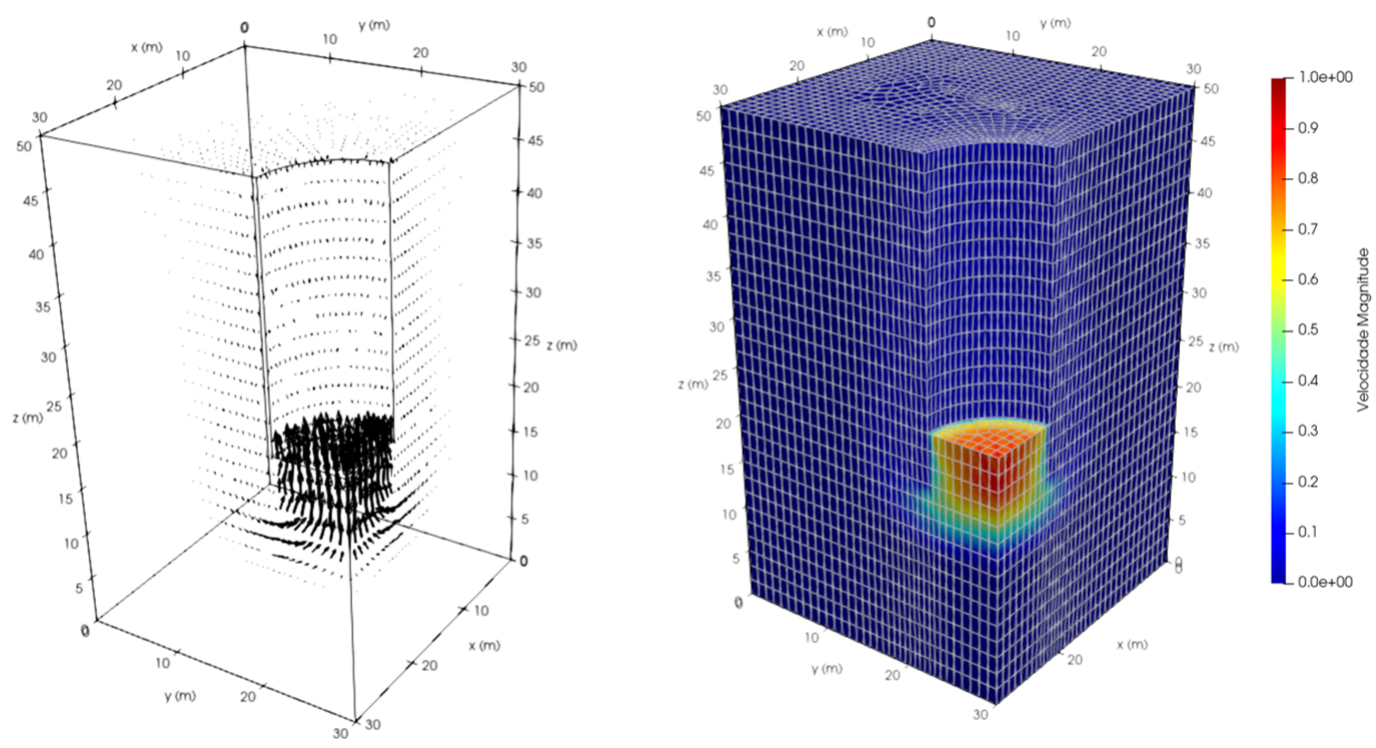

Figura 4.21: Mecanismo de colapso obtido na análise tridimensional, análise do poço com anel de concreto, SOCP D-P Cone 2, representação do caso 1.
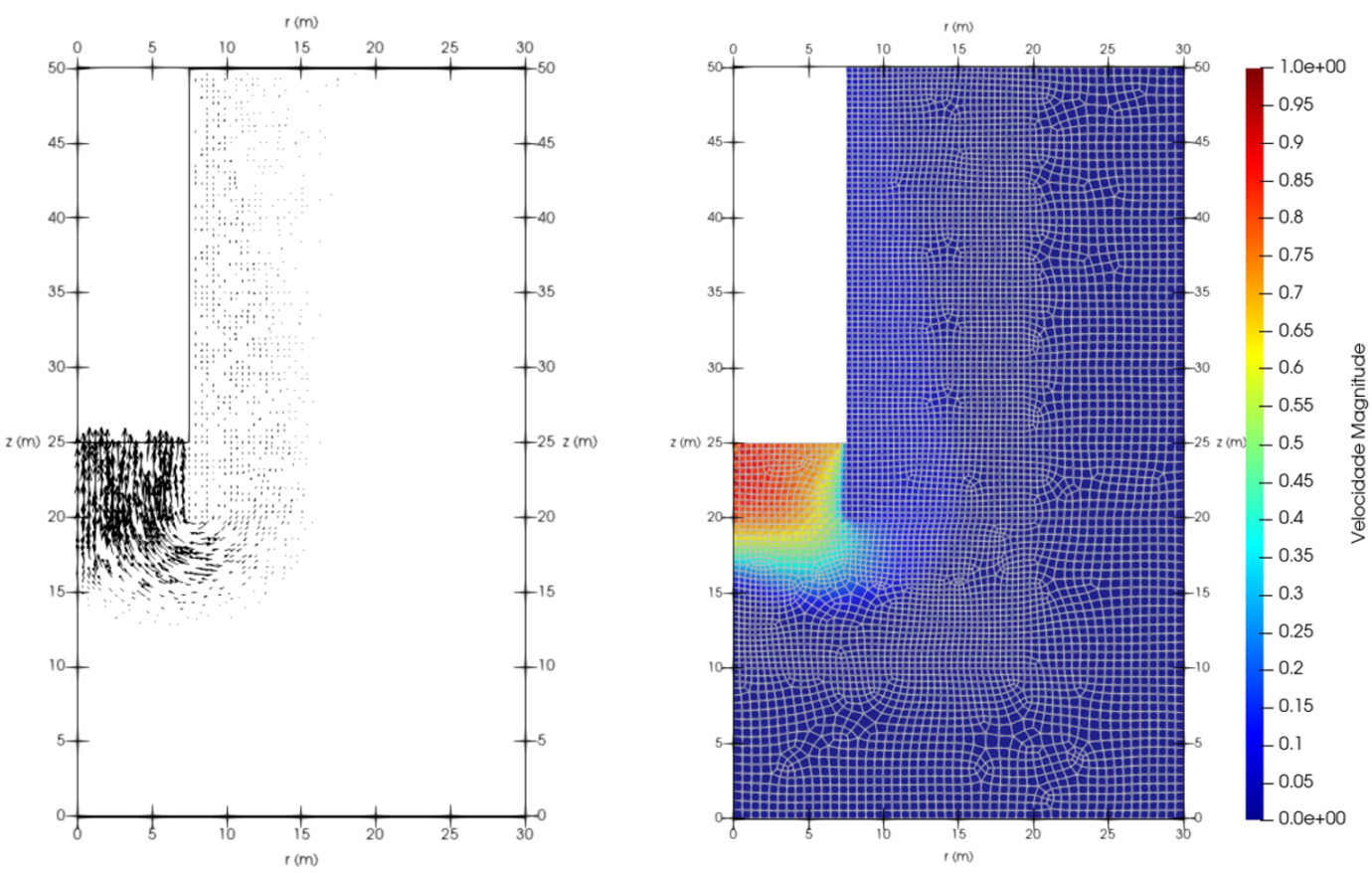

Figura 4.22: Mecanismo de colapso obtido na análise axissimétrica, análise do poço com anel de concreto, SOCP D-P Cone 2, representação do caso 1.

Cone 2 e SDP M-C, respectivamente.

- A diferença percentual media com respeito aos $F S$ obtidos de Plaxis 
2D foram de $4.83 \%, 6.43 \%$ e $0.75 \%$ para as analises SOCP D-P Cone 1 , Cone 2 e SDP M-C, respectivamente.

- As analises feitas com o Cone 1 D-P resultaram para todos os casos em estimações mais conservadoras do $F S$, nas analises feitas com o Cone 2 as estimações resultaram ligeiramente superiores aos obtidos dos softwares, e na aplicação direta do critério M-C com SDP se obtiveram os resultados mais aproximados aos conseguidos dos softwares, sendo que estes também utilizam o criterio M-C.

Os tempos médios de processamento foram, no caso da programação SOCP D-P de 14 segundos, na programação SDP de 253 segundos, em Optum G2 de 24 segundos e em Plaxis 2D de 30 segundos. Na Figura 4.24 são apresentados os mecanismos de colapso, que como se pode notar resultaram muito similares.

Tabela 4.12: Fator de segurança obtido no análise de estabilidade do poço com anel de concreto para diferentes casos de parâmetros do solo

\begin{tabular}{clcccccccc}
\hline \multirow{5}{*}{ Parâmetros } & Caso & 1 & 2 & 3 & 4 & 5 & 6 & 7 & 8 \\
\cline { 2 - 9 } & $\gamma\left(\mathrm{kN} / \mathrm{m}^{3}\right)$ & 18 & 16 & 17 & 17 & 18 & 17 & 17 & 19 \\
& $c(k P a)$ & 10 & 10 & 15 & 25 & 5 & 1 & 10 & 50 \\
& $\phi\left(^{\circ}\right)$ & 20 & 15 & 25 & 10 & 12 & 15 & 18 & 35 \\
\hline \multirow{5}{*}{ FS } & SOCP D-P Cone 1 & 2.77 & 2.13 & 3.62 & 1.93 & 1.59 & 1.84 & 2.51 & 6.18 \\
& SOCP D-P Cone 2 & 3.10 & 2.38 & 4.05 & 2.18 & 1.77 & 2.05 & 2.80 & 6.93 \\
& SDP M-C & 2.93 & 2.25 & 3.83 & 2.07 & 1.68 & 1.93 & 2.65 & 6.56 \\
& M-C Optum G2 & 2.89 & 2.22 & 3.77 & 2.04 & 1.66 & 1.88 & 2.62 & 6.48 \\
& M-C Plaxis & 2.91 & 2.23 & 3.80 & 2.04 & 1.68 & 1.92 & 2.61 & 6.58 \\
\hline
\end{tabular}

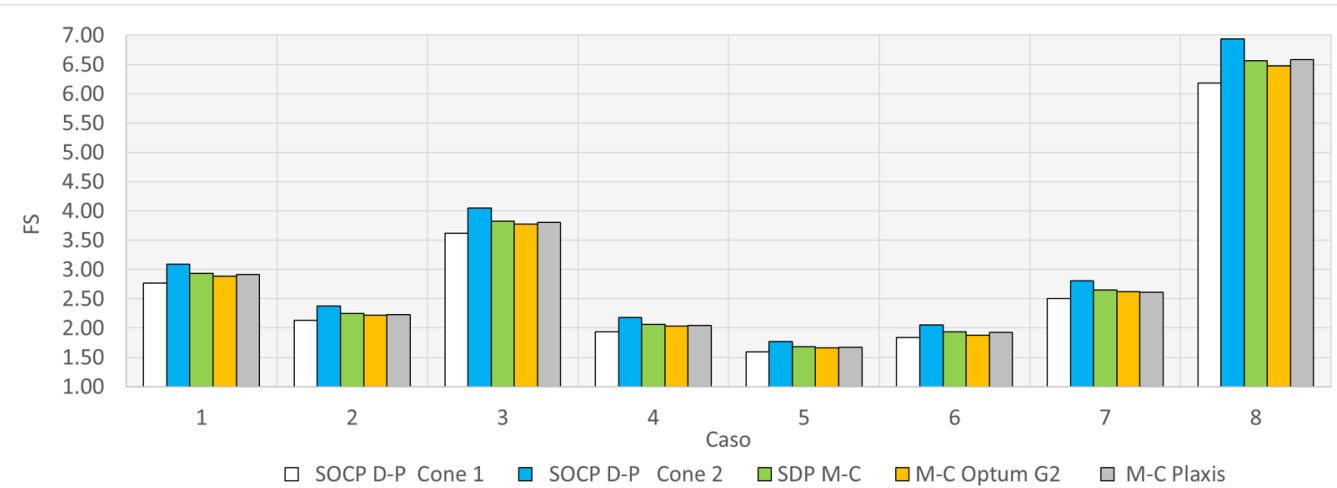

Figura 4.23: Comparação dos resultados conseguidos neste trabalho com os resultados obtidos em Optum G2 e Plaxis 2D. 


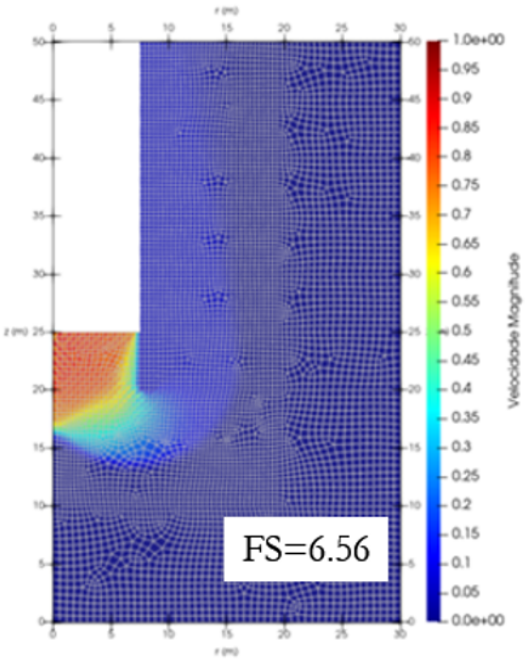

(a)

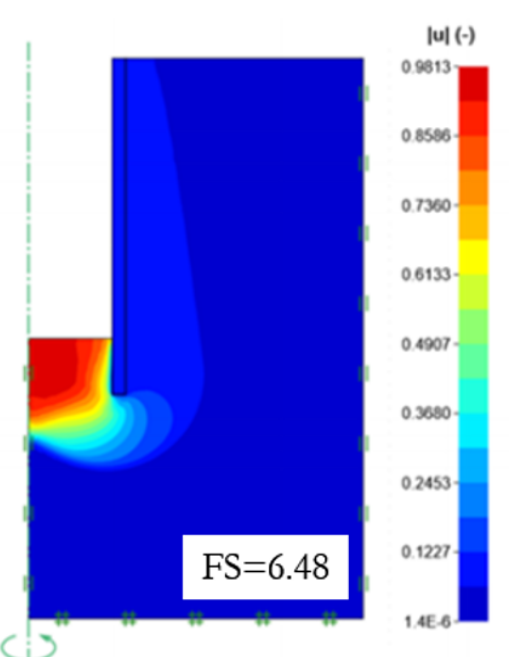

(b)

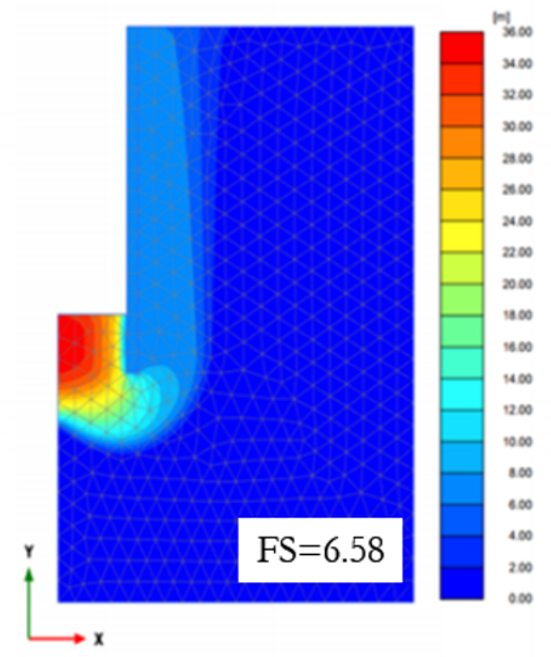

(c)

Figura 4.24: Mecanismo de colapso, poço com muro, caso 8. (a) Análise limite programação SDP M-C (b) Optum G2 (c) Plaxis 2D.

\subsubsection{Analise comparativo ALN-MPM}

De forma complementar na verificação dos resultados, o caso 8 dos parâmetros do solo da Tabela 4.12 foi analisado por Fernández [58] com o software MPM-PUCRio ${ }^{3}$, foi criado um modelo MPM tridimensional, formado por 93702 pontos materiais distribuídos numa malha Euleriana de células de $0.6 \mathrm{~m}$ de lado. A modelagem considerou o comportamento elastoplástico do material, implementando o critério de ruptura de Mohr-Coulomb sem amolecimento, com os parâmetros de resistência dos materiais apresentados na Tabela 4.13.

Tabela 4.13: Parâmetros de resistência dos materiais para a modelagem MPM

\begin{tabular}{lll|lll}
\hline \multicolumn{3}{c}{ Solo } & \multicolumn{3}{c}{ Concreto } \\
\hline$E$ & 500 & $M P a$ & $E$ & 20 & $G P a$ \\
$v$ & 0.2 & $(-)$ & $v$ & 0.2 & $(-)$ \\
$\gamma$ & 19 & $k N / m^{3}$ & $\gamma$ & 22 & $k N / m^{3}$ \\
$c$ & 50 & $k P a$ & & & \\
$\phi$ & 35 & $\circ$ & & & \\
\hline
\end{tabular}

Para determinar o FS na analise com MPM foi selecionado um ponto de controle no centro do poço $(x=0, y=0, z=25)$, o FS pode ser obtido

${ }^{3}$ MPM (Método do Ponto Material) é um método numérico, que pode ser utilizado na modelagem de problemas geotécnicos de grandes deformações 


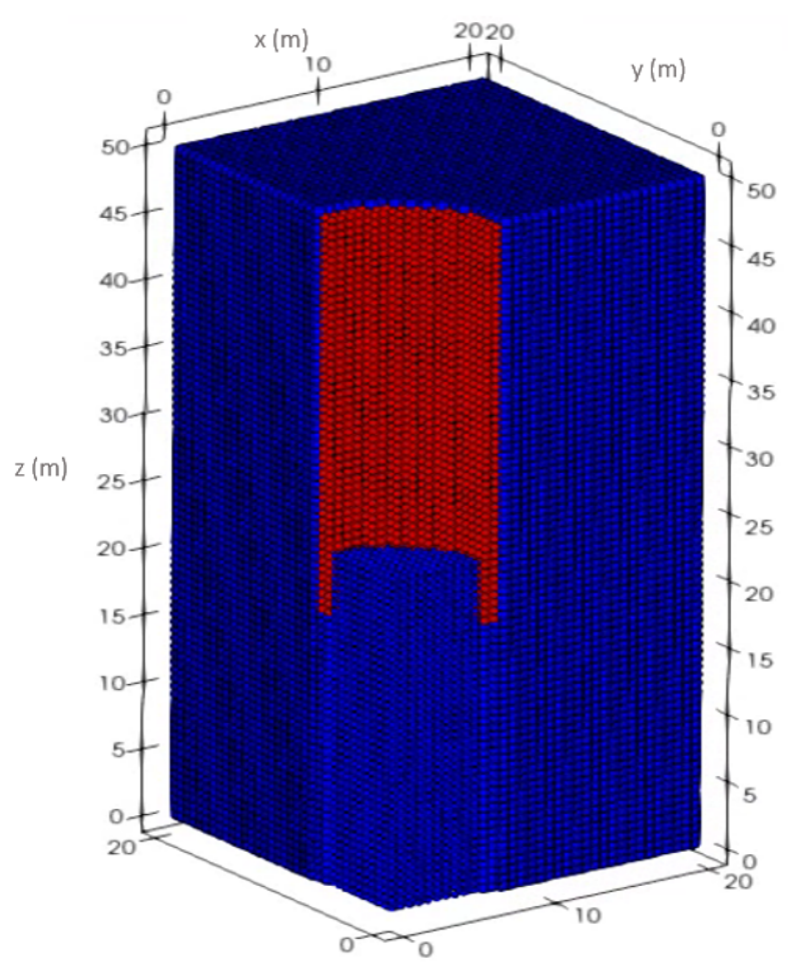

Figura 4.25: Modelo MPM tridimensional do poço com o anel de concreto, formado por 93702 pontos materiais.

graficamente como é representado na Figura 4.26. Da analise é estimado um $F S=6.5$, que resulta muito próximo aos valores conseguidos nesta pesquisa, sendo de $4.88 \%, 6.64 \%$, e $0.97 \%$ a diferença percentual com os resultados da programação SOCP D-P Cone 1, Cone 2 e SDP M-C respectivamente.

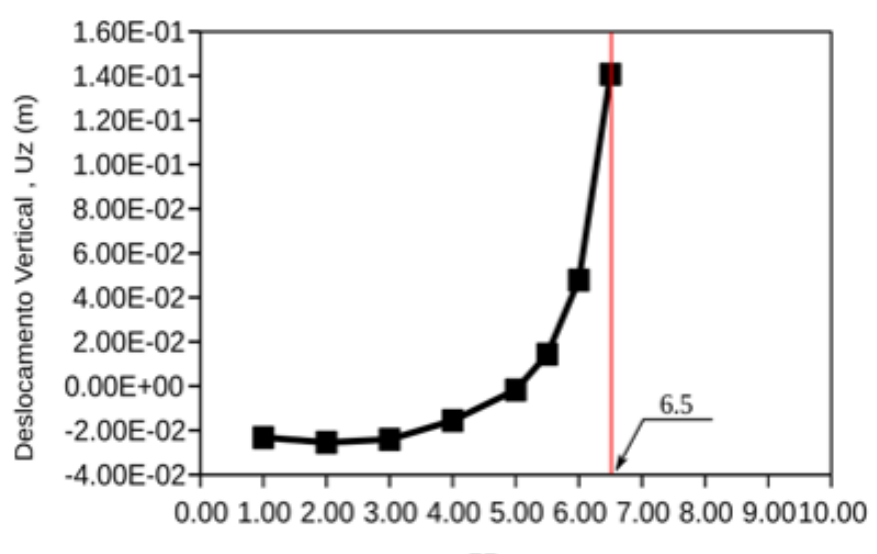

FR

Figura 4.26: Deslocamentos verticais finais para diversos FR, avaliados no ponto de controle no centro do poço. 
Na Figura 4.27, é apresentado o mecanismo de ruptura obtido na analise MPM, que de maneira similar ao mecanismo de colapso da Figura 4.24, estimado com ALN, reproduz a ruptura pela base ou fundo do poço.
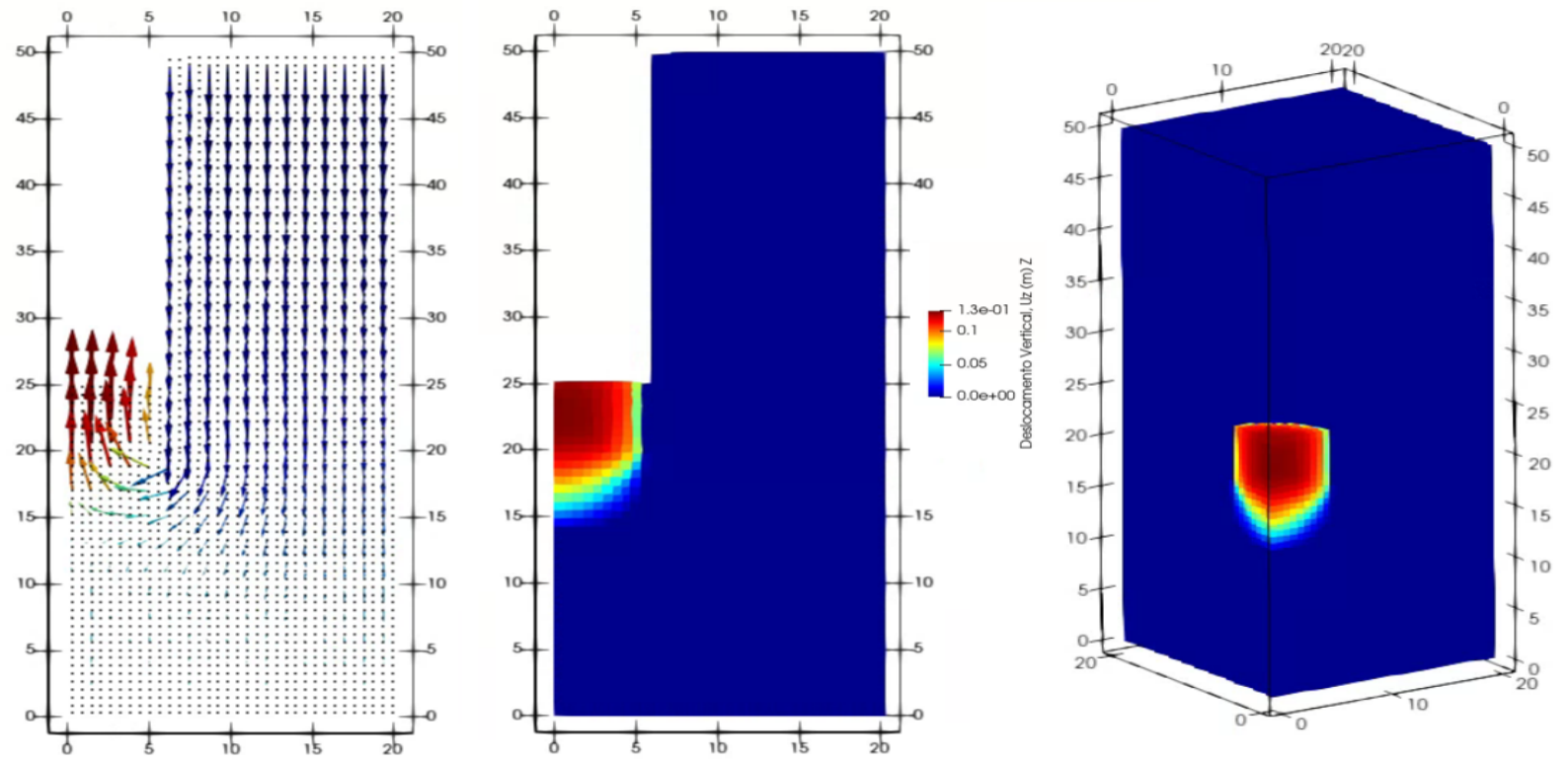

Figura 4.27: Mecanismo de ruptura obtido com MPM. As Figuras mostram o campo de deslocamentos verticais em metros, $\mathrm{FS}=6.5$.

\subsubsection{Analise de estabilidade do poço com anel de concreto, considerando heterogeneidade}

Para a fase 2 foi estudado o problema de heterogeneidade apresentado na Figura 4.28. Tem-se a presença de 3 camadas de solo, foram efetuadas analises do tipo SOCP D-P Cone 1 e 2 e SDP M-C, além do mais foi feito um analise em Plaxis 2D para a verificação dos resultados. As condições de contorno impostas são as mesmas da Tabela 4.10. Os parâmetros de resistência do solo e os $F S$ estimados são apresentados na Tabela 4.14.

Dos resultados da analise se pode observar que os obtidos com SDP MC e Plaxis 2D foram muito próximos, com a programação SOCP D-P segue apresentando-se a tendência de estimativas conservadoras com o Cone $1 \mathrm{e}$ maiores com o Cone 2. Os mecanismos de colapso obtidos são apresentados na Figura 4.29.

Para o mesmo exemplo com a finalidade de conferir a influência que tem na estabilidade global da estrutura as propriedades resistentes das 3 

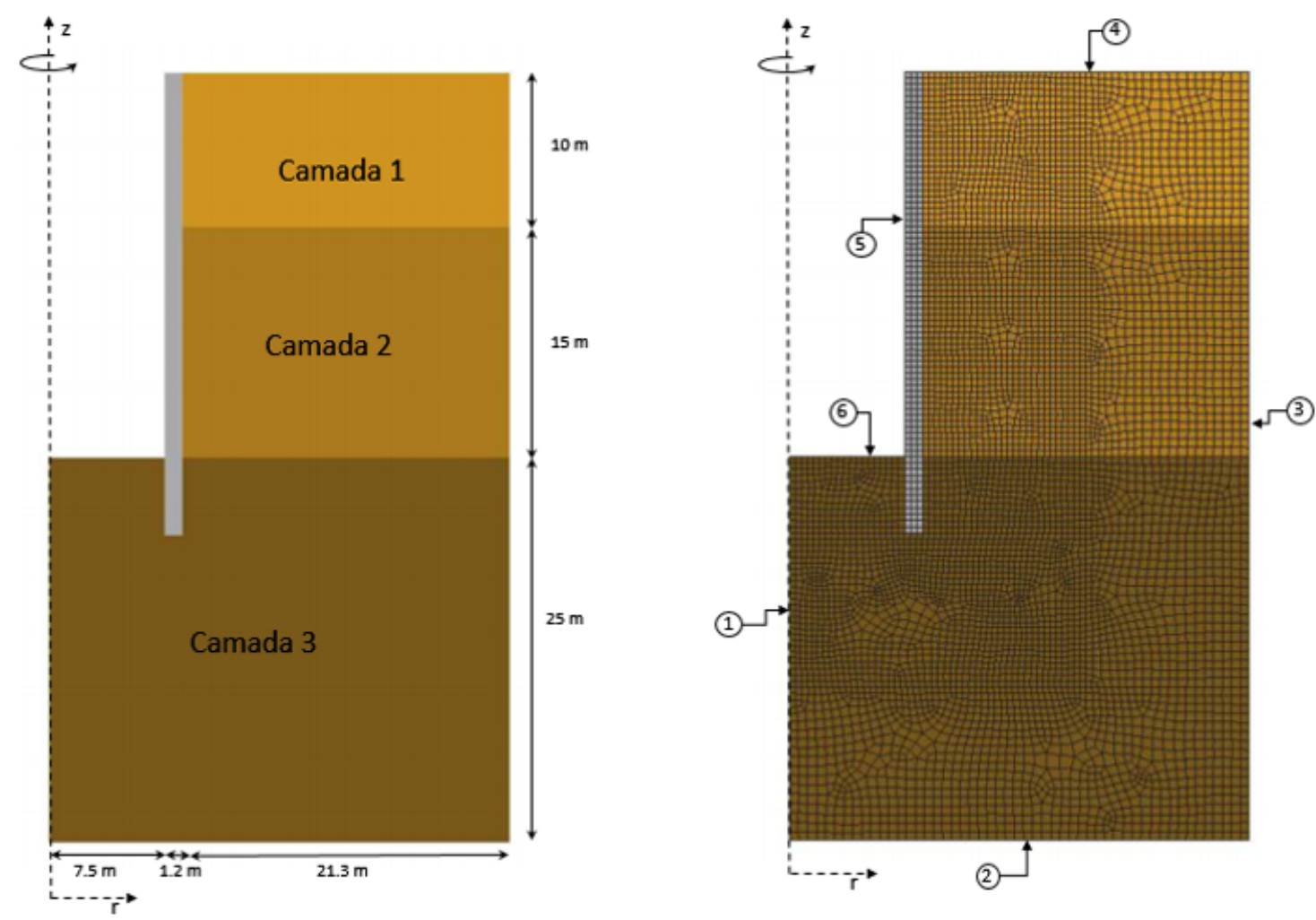

Figura 4.28: Geometria e malha não estruturada utilizada no modelo do poço com anel de concreto, considerando heterogeneidade.

Tabela 4.14: Parâmetros das camadas de solo e resultados do analise de estabilidade do poço

\begin{tabular}{|c|c|c|c|c|c|}
\hline \multirow{4}{*}{ Parâmetros } & \multicolumn{2}{|r|}{ Camada } & 1 & 2 & 3 \\
\hline & \multicolumn{2}{|c|}{ Peso específico $(\mathrm{kN} / \mathrm{m} 3)$} & 16 & 17 & 18 \\
\hline & \multicolumn{2}{|c|}{ Coesão $(\mathrm{kPa})$} & 1 & 2 & 4 \\
\hline & \multicolumn{2}{|c|}{ Ângulo de atrito $\left(^{\circ}\right)$} & 10 & 15 & 22 \\
\hline Tipo de analise & FS & Tempo de processamento (s) & Número de nós & Dif & $\begin{array}{l}\text { percentual } \\
\text { SD }\end{array}$ \\
\hline SOCP D-P Cone 1 & 2.73 & 8.29 & 6443 & & \\
\hline SOCP D-P Cone 2 & 3.04 & 10.37 & 6443 & & \\
\hline SDP M-C & 2.87 & 194.58 & 6443 & & \\
\hline MC Plaxis & 2.90 & 42.00 & 6831 & & \\
\hline
\end{tabular}

camadas, foi efetuado um analise adicional com programação SOCP D-P Cone 2:

- Partindo dos parâmetros de resistência do solo da Tabela 4.14, foram reduzidos um $50 \%$ os valores da coesão $(c)$ e ângulo de atrito $(\phi)$ das camadas 1 e 2, sem modificar os parâmetros da camada 3. Sob essas condições o $F S$ estimado foi de 2.81

- Alternativamente, e com base também na Tabela 4.14, foram reduzidos 


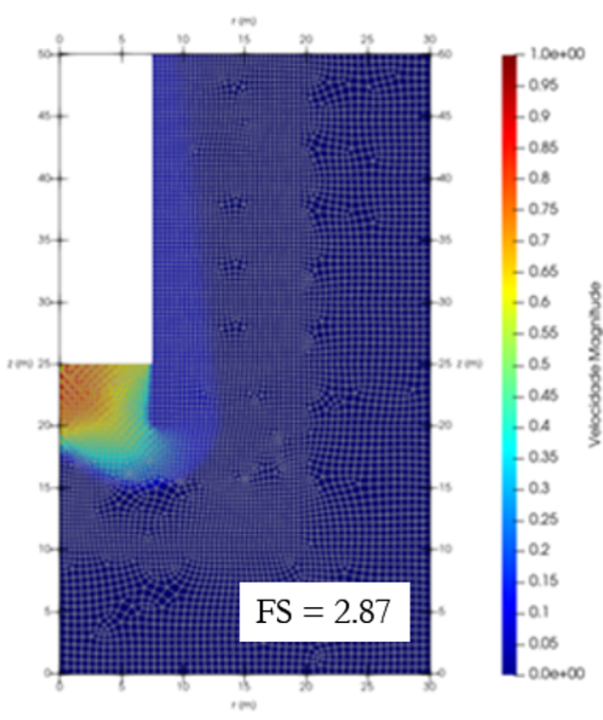

(a)

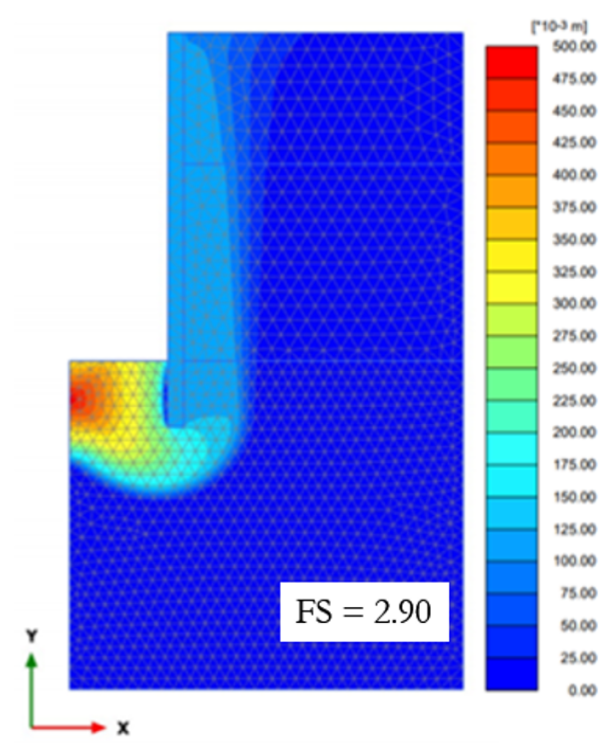

(b)

Figura 4.29: Mecanismo de colapso obtido para o analise de heterogeneidade da Fase 2 (a) Análise limite com programação SDP M-C (b) Plaxis 2D.

um 50\% os valores da coesão $(c)$ e ângulo de atrito $(\phi)$ da camada 3, sem modificar os parâmetros das camadas 1 e 2 . Sob essas condições o $F S$ estimado foi de 1.64

Visto que o FS obtido com os parâmetros originais foi de 3.04, a redução de um $50 \%$ dos parâmetros das camadas 1 e 2 somente representou uma redução de um $7.72 \%$ do $F S$, enquanto a redução do $50 \%$ dos parâmetros da camada 3 significou a redução de um $46.06 \%$ do $F S$. Sendo assim é possível concluir que o aporte à resistência da camada 3 é fundamental na analise de estabilidade do poço, para o mecanismo de colapso estudado.

\subsection{Fase 3: Analise de estabilidade de poço com anel de concreto, considerando a presença do nível da água}

A fase final do problema estudado incorpora na fase anterior a presença do nível da água, e considera portanto, seu efeito na analise de estabilidade. Para isto foram executadas analises SOCP D-P, programação para a qual foi estudada a inclusão do efeito das poropressões na secção 3.4.2. A equação de equilíbrio considerada teve a mesma forma que a equação (4-3) da Fase 2.

As analises são divididas em duas etapas: A analise de fluxo para obtenção das poropressões e a analise de estabilidade. A analise de fluxo foi feita no Optum G2 e as poropressões calculadas pelo software foram expor- 
tadas e interpoladas para os elementos da malha do analise de estabilidade, de tal forma que no centroide de cada elemento foi definido um determinado valor de poropressão. Na Figura 4.30 é apresentada a geometria, e a distribuição das poropressões definida na malha não estruturada do problema. $\mathrm{O}$ solo acima do nível da àgua foi considerado seco.

Foram efetuadas 5 analises, para solos com diferentes parâmetros de resistência, porém com o mesmo valor de condutividade hidráulica $k$, o concreto foi considerado como material impermeável e seus parâmetros de resistência foram os mesmos obtidos da analise de calibração da fase 2 . Na Tabela 4.15 são apresentados os parâmetros do solo e os resultados obtidos.
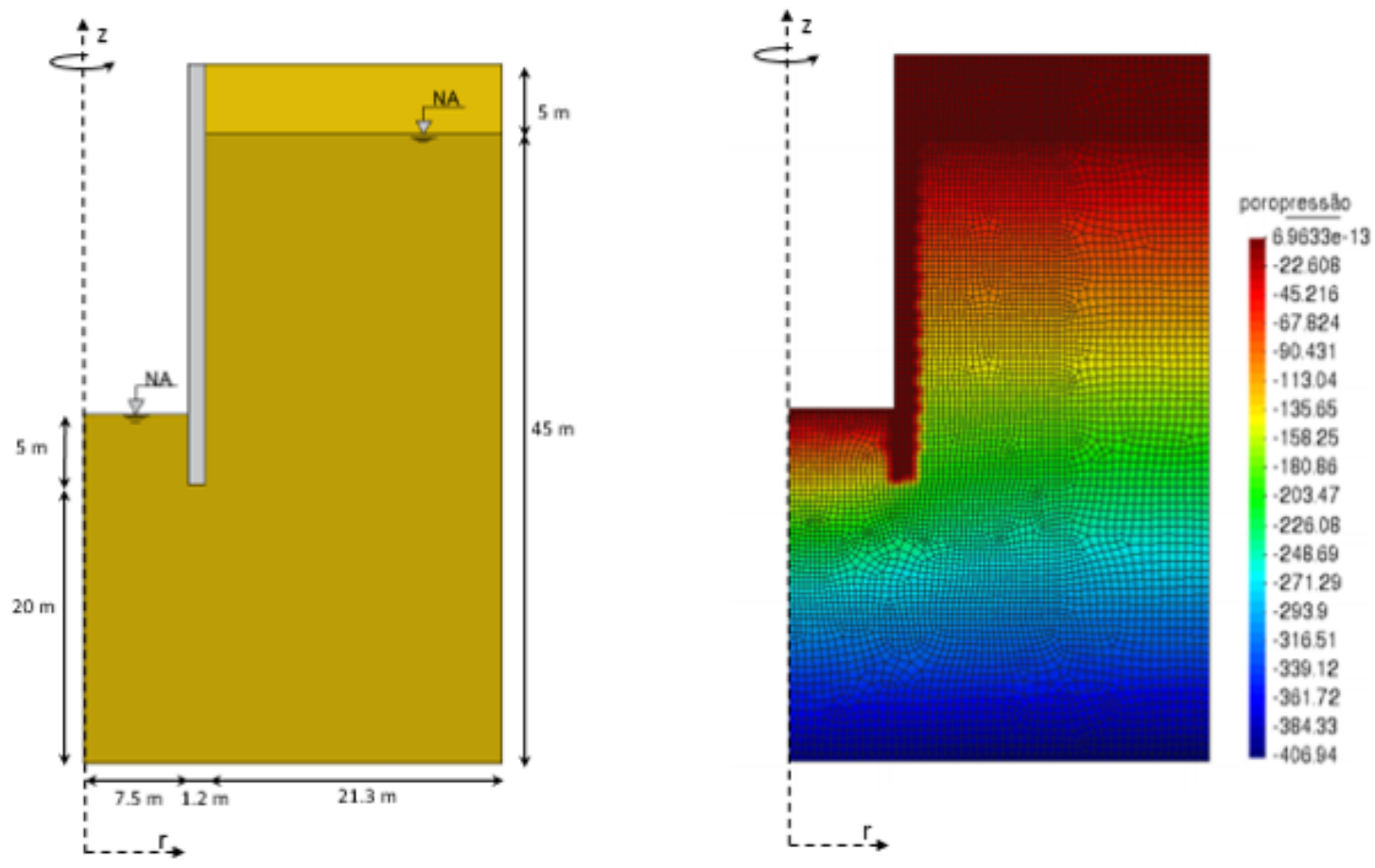

Figura 4.30: Distribuição das poropressões ( $\mathrm{kPa}$ ) adotada na análise.

Dos resultados das analises se pode reparar na diminuição geral do $F S$ calculado ao incluir o efeito das poropressões,especificamente no caso 5 , análogo ao caso 8 da fase 2 (Tabela 4.12) pode-se estimar uma redução do $F S$ de um 50\% aproximadamente. A diferença percentual media dos analises respeito aos resultados de Optum G2 foi de 3.96\% e $5.66 \%$ para o SOCP D$\mathrm{P}$ Cone 1 e Cone 2 respectivamente. $\mathrm{O}$ tempo médio de processamento da programação SOCP D-P foi de 12 segundos e do Optum G2 de 15 segundos.

Na Figura 4.31 são apresentados os mecanismos de ruptura obtidos das 
Tabela 4.15: Fator de segurança obtido na análise de estabilidade do poço com muro, considerando poropressão, para diferentes casos de parâmetros do solo

\begin{tabular}{clccccc}
\hline \multirow{5}{*}{ Parâmetros } & Caso & 1 & 2 & 3 & 4 & 5 \\
\cline { 2 - 7 } & $\gamma_{d}\left(k N / m^{3}\right)$ & 16 & 17 & 16 & 18 & 19 \\
& $\gamma_{s a t}\left(k N / m^{3}\right)$ & 18 & 18 & 17 & 19 & 20 \\
& $c(k P a)$ & 10 & 15 & 25 & 40 & 50 \\
& $\phi\left({ }^{\circ}\right)$ & 20 & 25 & 25 & 20 & 35 \\
& $k(m / s)$ & $1.50 \mathrm{E}-05$ & $1.50 \mathrm{E}-05$ & $1.50 \mathrm{E}-05$ & $1.50 \mathrm{E}-05$ & $1.50 \mathrm{E}-05$ \\
\hline \multirow{3}{*}{ FS } & SOCP D-P Cone 1 & 0.43 & 0.70 & 1.30 & 1.96 & 3.05 \\
& SOCP D-P Cone 2 & 0.45 & 0.73 & 1.30 & 2.16 & 3.33 \\
& M-C Optum G2 & 0.48 & 0.74 & 1.28 & 2.00 & 3.02 \\
\hline
\end{tabular}

analises.

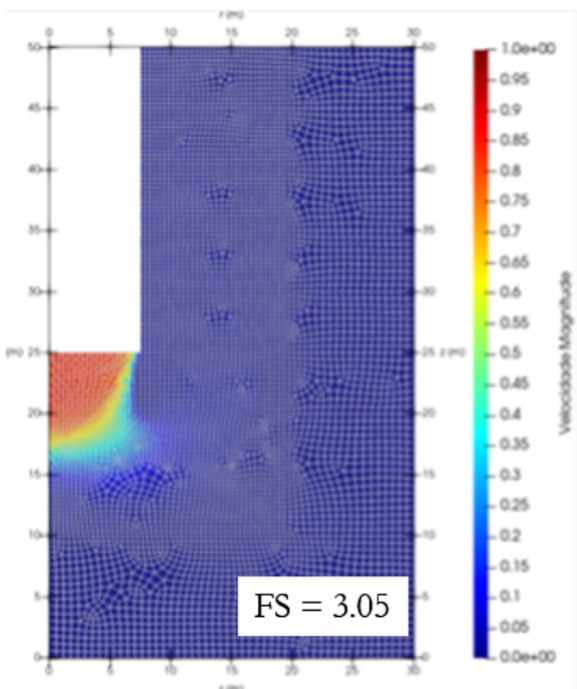

(a)

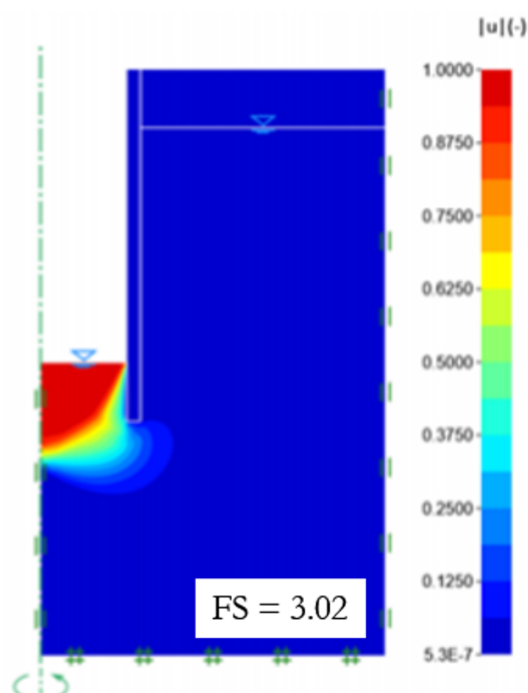

(b)

Figura 4.31: Mecanismo de colapso, análise do poço com muro considerando o efeito da poropressão, caso 5. (a) Análise limite com programação SOCPDP Cone 1 (b) Optum G2.

\subsubsection{Analise comparativo ALN-MPM}

O problema da fase 3 também foi analisado por Fernández [58], mediante um modelo MPM tridimensional, considerando os parâmetros dos materiais apresentados na Tabela 4.16.

O FS foi obtido graficamente com ajuda de um ponto de controle no meio do poço $(x=0, y=0, z=25)$, como é representado na Figura 4.32.

O FS estimado no analise MPM foi de 3, sendo de $1.67 \%$ e $11 \%$ a 
Tabela 4.16: Parâmetros dos materiais para a modelagem MPM

\begin{tabular}{lll|lll}
\hline & \multicolumn{3}{c}{ Solo } & \multicolumn{3}{c}{ Concreto } \\
\hline $\mathrm{E}$ & 500 & $M P a$ & $\mathrm{E}$ & 20 & $G P a$ \\
$\mathrm{v}$ & 0.2 & $(-)$ & $\mathrm{v}$ & 0.2 & $(-)$ \\
$\gamma_{d}$ & 19 & $k N / m^{3}$ & $\gamma$ & 22 & $k N / \mathrm{m}^{3}$ \\
$\gamma_{\text {sat }}$ & 20 & $k N / \mathrm{m}^{3}$ & & & \\
$c$ & 50 & $k P a$ & & & \\
$\phi$ & 35 & $\circ$ & & & \\
$k$ & $1.50 \mathrm{E}-05$ & $\mathrm{~m} / \mathrm{s}$ & & & \\
\hline
\end{tabular}

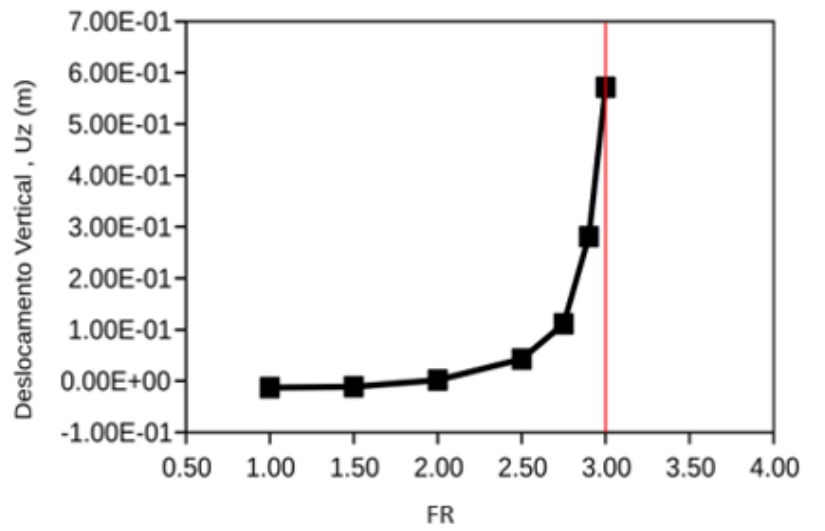

Figura 4.32: Deslocamentos verticais finais para diversos FR, avaliados no ponto de controle no centro do poço.

diferença percentual com os resultados da programação SOCP D-P Cone 1 e Cone 2 respectivamente. $\mathrm{O}$ mecanismo de ruptura identificado na analise MPM é apresentado na Figura 4.33. 

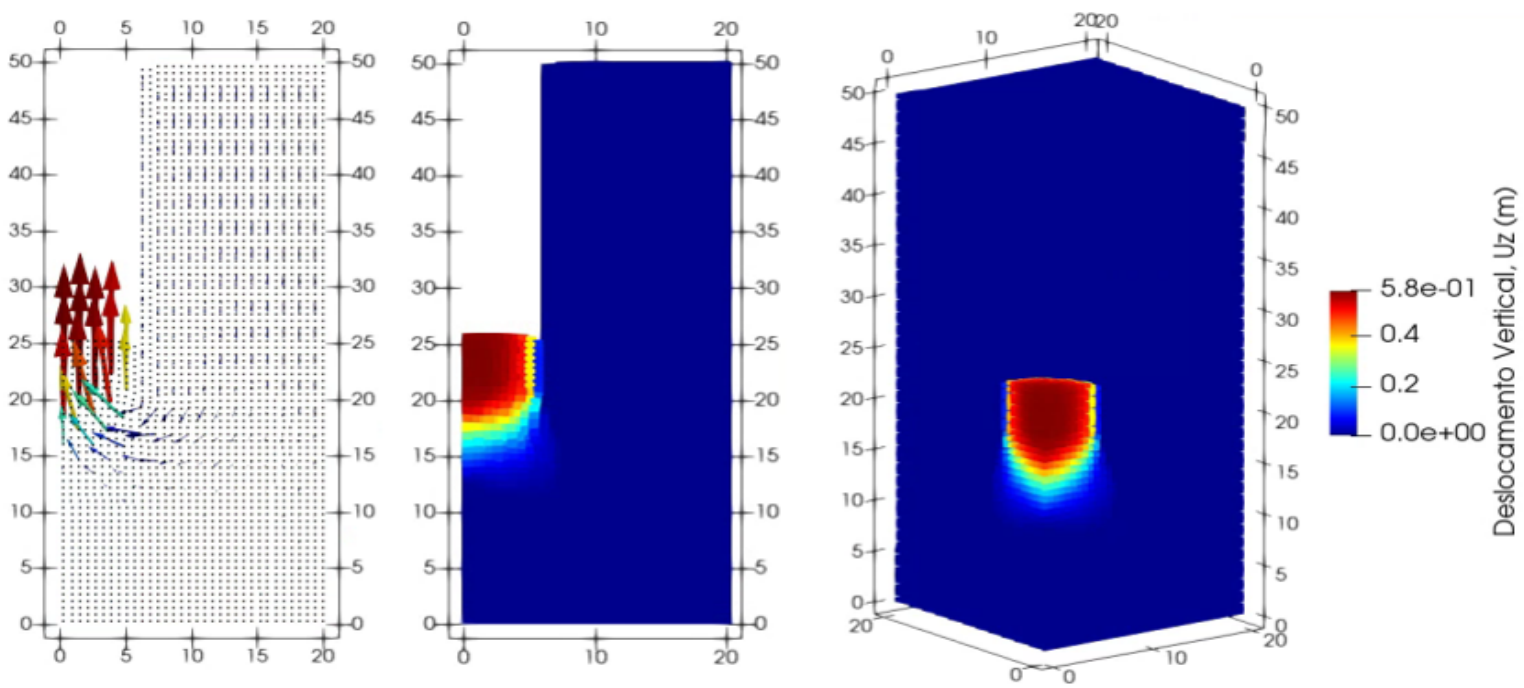

Figura 4.33: Mecanismo de ruptura obtido com MPM. As Figuras mostram o campo de deslocamentos verticais em metros, $\mathrm{FS}=3.0$.

\subsection{Analise da capacidade de carga de uma fundação circular rasa}

Um assunto de interesse na prática da engenharia, é a estimação da capacidade de carga de fundações, na condição axissimétrica pode-se estudar o caso de fundações circulares rasas dando mais uma aplicação ao código implementado neste trabalho. Foram executadas analises comparativas em termos do fator de capacidade de carga $N_{c}$, o qual pode-se obter da equação de capacidade de carga para uma sapata circular rasa:

$$
q_{u}=c N_{c}+\frac{1}{2} D \gamma N_{\gamma}
$$

onde $q_{u}$ e a capacidade de carga (Força de superfície) obtida da ALN, $c$ e $\gamma$ são os parâmetros de resistência do material, $D$ é o diâmetro da fundação e $N_{\gamma}$ e $N_{c}$ são os fatores de capacidade de carga que dependem do valor do ângulo de atrito $\phi$. Assumindo um valor de coesão $c=1 k P a$ e do peso específico $\gamma=0$, a equação (4-4) pode-se reduzir a:

$$
q_{u}=N_{c}
$$

Dessa maneira é estimado o valor de $N_{c}$. Em quanto à determinação de $q_{u}$, neste trabalho se optou por seguir a seguinte metodologia:

- O modelo adotado e apresentado na Figura 4.34, considera a presença de dois materiais, solo e concreto, o concreto correspondente à sapata 
de raio $R=1 \mathrm{~m}$ e espessura $e=0.3 \mathrm{~m}$. As condições de contorno impostas são apresentadas na Tabela 4.17.

Tabela 4.17: Condições de contorno no modelo axissimétrico da sapata circular rasa

\begin{tabular}{llc}
\hline \multirow{2}{*}{ Contorno } & \multicolumn{2}{c}{ Restrito em } \\
\cline { 2 - 3 } & r & Z \\
\hline 1 & $\checkmark$ & \\
2 & $\checkmark$ & $\checkmark$ \\
3 & $\checkmark$ & \\
4 & & \\
\hline
\end{tabular}

- Para os elementos pertencentes à sapata foram restritos os graus de liberdade em $r$, e considerados os parâmetros de resistência $\phi=60^{\circ}$, $c=2000 \mathrm{kPa}$ e $\gamma=1 \mathrm{kN} / \mathrm{m}^{3}$, o valor do peso específico foi assumido assim, já que a ideia para a aplicação da carga foi maximizar o peso específico da sapata

- A equação de equilíbrio foi modificada da seguinte maneira:

$$
[G]\{\sigma\}=\lambda\left\{f_{w c}\right\}+\left\{f_{w s}\right\}
$$

onde o vetor maximizado $\left\{f_{w c}\right\}$ é um vetor de força de volume, correspondente ao peso específico do concreto e $\left\{f_{w s}\right\}$ é o vetor de força de volume do solo. O resultado do processo de otimização é uma carga por unidade de volume, para obter $q_{u}$ esse valor foi multiplicado pela espessura da sapata $(e)$.

Para as analises foram utilizados todos os Cones do critério D-P com programação SOCP, e o criterio M-C com SDP. Na Tabela 4.18 são apresentados os resultados obtidos neste trabalho e os obtidos por outros autores.

Tabela 4.18: Comparação dos valores do fator de capacidade de carga $N_{c}$ obtidos para uma fundação circular rasa

\begin{tabular}{|c|c|c|c|c|c|c|c|c|}
\hline \multirow{2}{*}{$\phi\left(^{\circ}\right)$} & \multicolumn{6}{|c|}{ RESULTADOS OBTIDOS NESTE TRABALHO } & \multirow{2}{*}{$\begin{array}{c}\text { Sun e Yang } \\
(2020) \\
\text { LI }\end{array}$} & \multirow{2}{*}{$\begin{array}{l}\text { Mohapatra } \\
\text { e Kummar } \\
\text { (2018) } \\
\text { LS }\end{array}$} \\
\hline & $\begin{array}{ll}\text { SOCP } & \text { D-P } \\
\text { cone } 1 & \end{array}$ & $\begin{array}{ll}\text { SOCP } & \text { D-P } \\
\text { cone } 2 & \end{array}$ & $\begin{array}{l}\text { SOCP D-P } \\
\text { cone } 3\end{array}$ & $\begin{array}{ll}\text { SOCP } & \text { D-P } \\
\text { cone } 4 & \end{array}$ & $\begin{array}{ll}\text { SOCP } & \text { D-P } \\
\text { cone } 5 & \end{array}$ & SDP M-C & & \\
\hline 5 & 7.00 & 8.12 & 8.32 & 8.43 & 8.76 & 8.05 & 8.05 & 8.12 \\
\hline 10 & 9.00 & 10.43 & 10.44 & 11.45 & 12.68 & 11.07 & 11.07 & 11.07 \\
\hline 15 & 11.71 & 13.51 & 14.44 & 16.08 & 19.70 & 15.82 & 15.81 & 15.83 \\
\hline 20 & 15.41 & 17.63 & 20.48 & 23.49 & 33.86 & 23.63 & 23.59 & 23.68 \\
\hline 25 & 20.50 & 23.19 & 30.24 & 36.16 & 68.42 & 37.24 & 37.21 & 37.36 \\
\hline 30 & 27.58 & 30.77 & 47.40 & 59.85 & 188.91 & 62.56 & 62.26 & 62.93 \\
\hline 35 & 37.49 & 41.20 & 81.38 & 110.65 & - & 113.72 & 112.46 & 114.78 \\
\hline 40 & 51.48 & 55.72 & 163.79 & 250.04 & - & 229.76 & 225.53 & 230.87 \\
\hline
\end{tabular}

Dos resultados obtidos se podem fazer as seguintes considerações: 


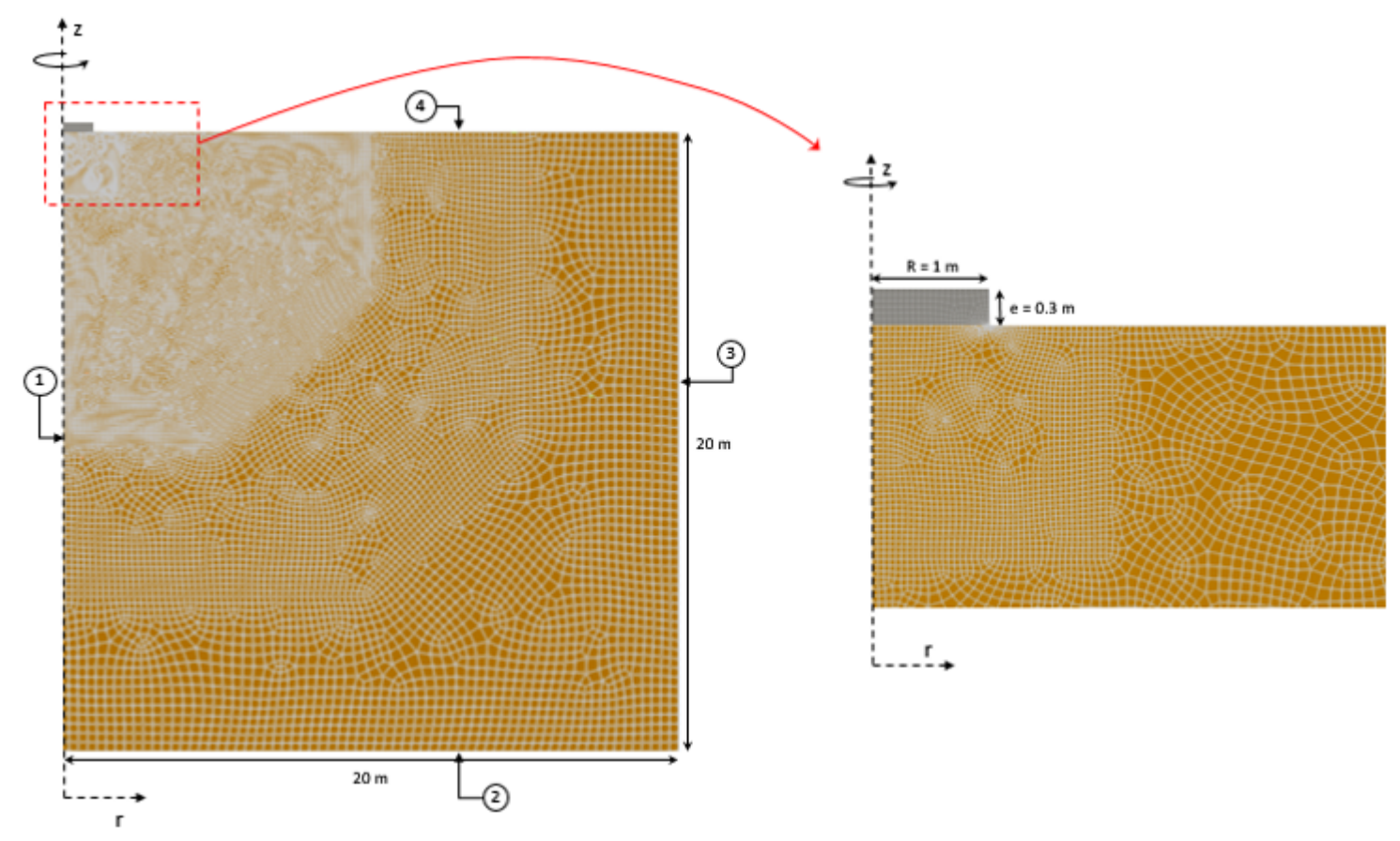

Figura 4.34: Geometria e malha não estruturada de 17685 elementos empregada na analise da capacidade de carga da sapata circular rasa.

- Os valores de $N_{c}$ obtidos com SDP M-C foram muito próximos aos obtidos por Sun e Yang ${ }^{4}$ e Mohapatra e Kummar ${ }^{5}$, sendo de somente $0.48 \%$ e $0.43 \%$ a diferença percentual media, respectivamente.

- Das analises feitas com os Cones D-P. As efetuadas com o Cone 1 e 2 resultaram em uma subestimação considerável do valor de $N_{c}$, sendo de $42.11 \%$ e $34.16 \%$ a diferença percentual media com os resultados de Sun e Yang.

- A melhor aproximação feita com os Cones D-P ao critério M-C com SDP e aos resultados de outros autores foi conseguida com o Cone 4, sendo de $3.68 \%$ e $3.70 \%$ a diferença percentual media com os resultados de Sun e Yang e Mohapatra e Kummar, respectivamente.

Na Figura 4.35 são apresentadas as superfícies de ruptura,definidas pelo campo de velocidades, para diferentes ângulos de atrito.

\footnotetext{
${ }^{4}$ Analise com formulação rigorosa pelo limite inferior e programação SDP M-C

${ }^{5}$ Analise com formulação rigorosa pelo limite superior e programação SDP M-C
} 

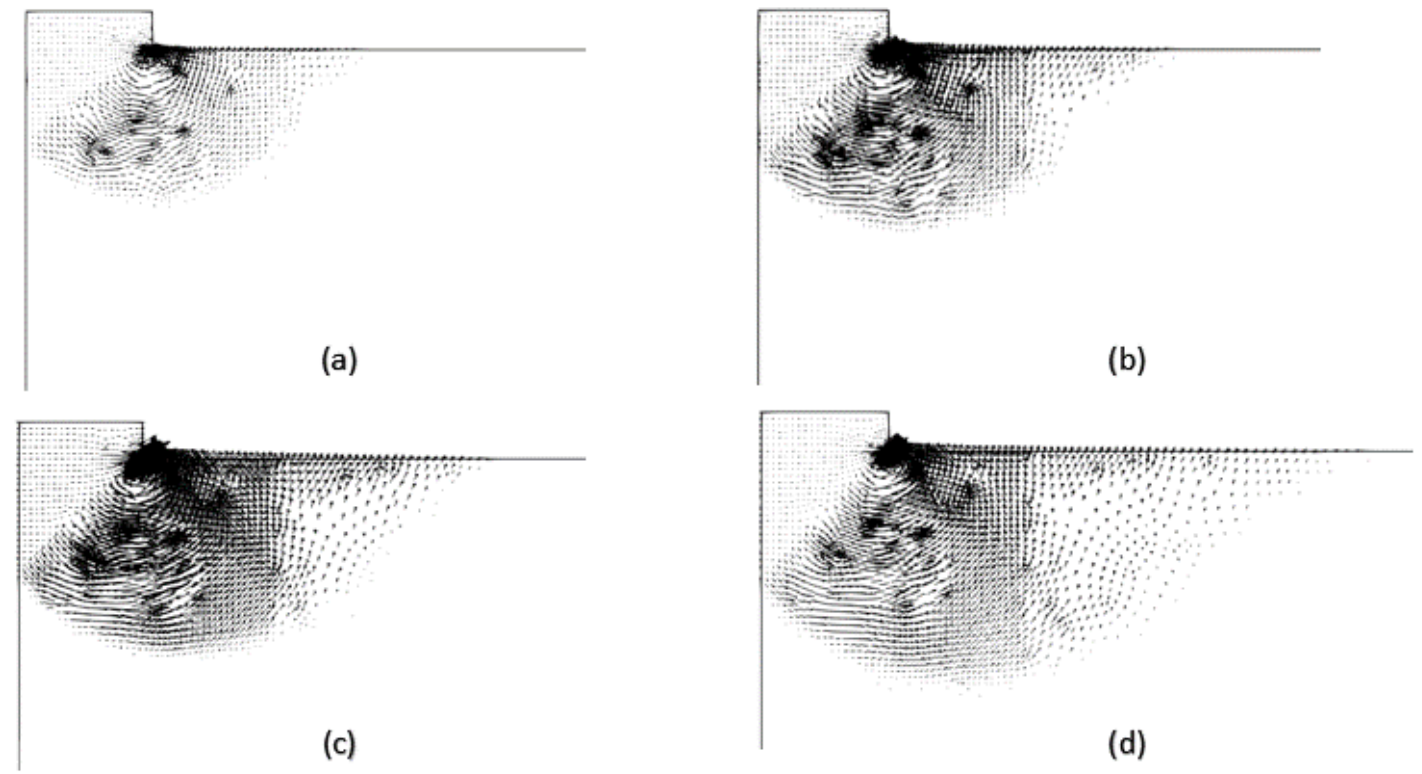

(d)

Figura 4.35: Superfícies de ruptura identificadas para (a) $\phi=15^{\circ}$ (b) $\phi=20^{\circ}$ (c) $\phi=25^{\circ}$ (d) $\phi=30^{\circ}$. 


\section{Conclusões}

O trabalho desenvolvido nesta dissertação de mestrado foi focado na aplicação da ALN para a resolução de problemas na condição axissimétrica. Dos resultados obtidos se podem fazer as seguintes conclusões:

- A comparação dos resultados da modelagem axissimétrica com a tridimensional, feita com SOCP D-P nas fases 1 e 2 do poço, demostrou a validez dos resultados obtidos, tanto em termos do $F S$ quanto na identificação do mecanismo de colapso, para o modelo plano com analise axissimétrica e sua eficiência em termos do tempo de processamento.

- Para a formulação mista implementada nesta dissertação, a sensibilidade do $F S$ ao processo de refinamento da malha foi baixa, com diferenças percentuais de menos do $1 \%$ em analises comparativas feitas aumentando em mais de 7 vezes o número de elementos da malha. Em concordância com o trabalho de Krabbenhøft et al. [9] no qual é demonstrado o desempenho da formulação mista e sua baixa variação para analises com malhas mais refinadas.

- Na fase 1 da análise de estabilidade do poço para os diferentes casos de parâmetros do solo, os Cones 1 e 2 do critério D-P demonstraram resultados mais próximos aos conseguidos da aplicação direta do critério M-C com programação SDP, sendo estes empregados nas analises das fases seguintes, com o Cone 1 foram obtidas estimações conservadoras do $F S$ e com o Cone 2 estimações ligeiramente superiores.

- Os tempos de processamento da programação SDP para todos os analises efetuados neste trabalho, com modelos planos no estado axissimétrico, foram superiores aos requeridos pela programação SOCP e a analise elastoplástica do Plaxis 2D. Por outro lado a programação SOCP demonstrou uma notável eficiência para o tratamento desse tipo de problemas, em geral as analises feitas com essa programação foram efetuadas em menos de 25 segundos.

- As analises efetuadas considerando heterogeneidade no poço, tanto na Fase 1 quanto na Fase 2, permitiram conferir a grande influência que 
tem a presença de camadas mais ou menos resistentes, na estimação do $F S$ e na definição da superfície de ruptura.

- Para a geometria do poço, na Fase 1, foi evidente que a estrutura geotécnica precisava de parâmetros do solo relativamente altos, que garantiram $F S$ ligeiramente superiores a 1, a inclusão de um anel de concreto no modelo, na Fase 2, representou um acréscimo considerável na estabilidade do poço, que sem ele, para a maioria de parâmetros de solo estudados, apresentou um estado de colapso iminente.

- Os resultados obtidos na Fase 2 da analise do poço, permitem concluir que o processo de calibração dos parâmetros $\mathrm{M}-\mathrm{C}$ do concreto, como material rígido foi bem sucedido, e garante o estudo de estabilidade diante da ruptura do solo.

- Como era esperado, a incorporação do efeito das poropressões no modelo do poço, na Fase 3, resultou na redução importante dos $F S$, sendo de aproximadamente um 50\% em comparação com as analises da Fase 2.

- Os resultados obtidos por Fernández [58] com o método MPM para as Fases 2 e 3 da analise do poço, foram muito próximos aos obtidos neste trabalho, na estimação do $F S$ e na identificação do mecanismo de colapso.

- As analises efetuadas para poços de diferentes proporções Raio/Profundidade, em termos do número de estabilidade $N_{s}$ permitiram verificar a boa aproximação feita pela formulação mista-fraca deste trabalho, às formulações rigorosas utilizadas por outros autores.

- Finalmente, no estudo da capacidade de carga, feito em termos do fator $N_{c}$, para uma fundação circular rasa, se verificou que a metodologia utilizada, com programação SDP M-C, permite obter resultados praticamente idênticos aos apresentados por Sun e Yang [16]. E no caso do critério D-P a melhor aproximação a esses resultados foi conseguida da aplicação do Cone 4 .

Como sugestões para futuras pesquisas tem-se:

- Para o estudo de estabilidade de maciços rochosos se sugere fazer a implementação com programação semidefinida do critério HoekBrown. Na literatura já tem-se referência da adaptação feita por Kumar e Mohapatra [59]. 
- Neste trabalho foi considerada a presença de um elemento de concreto, porém não se utilizaram elementos de interface na analise, seria interessante incorporá-los, como referência tem-se o trabalho de Ribeiro et al. [60] feito com programação não linear. Assim também sería de grande utilidade a inclusão de outros elementos de reforço no modelo, como por exemplo ancoragens.

- As analises da Fase 3 do poço, contemplando o efeito das poropressões foram executadas somente mediante o critério D-P, se sugere adaptar na analise M-C com programação semidefinida o efeito das poropressões. 


\section{Referências Bibliográficas}

[1] DAVIS, R. O; SElVADURAI, A. P. S. Plasticity and geomechanics. Cambridge:Cambridge University Press, 2002.

[2] PASTOR, J. Analyse limite: détermination numérique de solutions statiques complètes. application au talus vertical. Journal de Mécanique appliquée, 2(4):167-196, 1978.

[3] PASTOR, J; TUGERMAN, S. Limit analysis in axisymmetrical problems: Numeri- cal determination of complete statical solutions. International Journal of Mechanical Sciences, 24(2):95-117, 1982.

[4] ANDERHEGGEN, E; KNOPFEL, H. Finite element limit analysis using linear programming. International Journal of Solids and Structures, 8:1413-1431, 1995.

[5] SLOAN, S. Upper bound limit analysis using finite elements and linear programming. International Journal for Numerical and Analytical Methods in Geomechanics, 13:263-282, 1989.

[6] SLOAN, S; KLEEMAN, P. Upper bound limit analysis using discontinuous velocity fields. Computer Methods in Applied Mechanics and Engineering, 127:293-314, 1995.

[7] LYAMIN, A; SLOAN, S. Upper bound analysis using linear finite elements and non-linear programming. International Journal for Numerical and Analytical Methods in Geomechanics, 26:181-216, 2002b.

[8] KRABBENHØFT, K; DAMKILDE, L. A general non-linear optimization algorithm for lower bound limit analysis. International Journal for Numerical Methods in Engineering, 56:165-184, 2002.

[9] KRABBENHØFT, K; LYAMIN, A ; S.W., S. Formulation and solution of some plasticity problems as conic programs. International Journal of Solids and Structures, 44:1533-1549, 2007.

[10] MAKRODIMOPOULOS, A; MARTIN, C. Lower bound limit analysis of cohesive-frictional materials using second-order cone programming. 
International Journal for Numerical Methods in Engineering, 66:604-634, 2006.

[11] MOHAPATRA, D. Novel numerical procesdures for limit analysis: Implementation to planar, axisymetric and three-dimensional geomechanics stability problems. Tesis (Doutorado em Engenharia Civil)Department of Civil Engineering, Indian Institute of Science, 2020.

[12] TANG, C; TOH, K ; PHOON, K. Application of lower bound limit analysis with second order cone programming for plane strain and axisymmetric geomechanics problems. Journal of Engineering Mechanics, 140(2):268-278, 2014.

[13] KUMAR, J; KHATRI, V. Bearing capacity factors of circular foundations for a general $c-\phi$ soil using lower bound finite elements limit analysis. International Journal for Numerical and Analytical Methods in Geomechanics, 35(3):393-405, 2011.

[14] CHAKRABORTY, M; KUMAR, J. Lower-bound axisymmetric formulation for geomechanics problems using nonlinear optimization. International Journal of Geomechanics, 15, 2014.

[15] MOHAPATRA, D; KUMAR, J. Upper bound finite-element limit analysis of axisymmetric problems for mohr-coulomb materials using semidefinite programming. J. Eng. Mech., 144(7), 2018.

[16] SUN, R; YANG, J. Axisymmetric adaptive lower bound limit analysis for mohr-coulomb materials using semidefinite programming. Computers and Geotechnics, 2020.

[17] DRUCKER, D. On uniqueness in the theory of plasticity. Quaterly of Applied Mathematics, 14:35-42, 1956.

[18] HILL, R. The mathematical theory of plasticity. Oxford University Press, 7, 1998.

[19] CHEN, W. F. Limit analysis and soil plasticity developments in geotechnical engineering. 1975.

[20] CHEN, W. F; LIU, X. L. Limit analysis in soil mechanics. Elsevier, 1990.

[21] KIM, J; SALGADO, R. Limit analysis of soil slopes subjected to pore-water pressures. Journal of Geotechnical and Geoenvironmental Engineering, 125, 1999. 
[22] BARROS, G; PEREIRA, A ; VAZ, L. E. Análise limite com o método dos elementosde contorno. XXXV Iberian Latin-American Congress on Computational Methods in Engineering, CILAMCE 2014,Fortaleza, CE,Brazil:2326, 2014.

[23] CAMARGO, J. VELLOSO, R. Q; VARGAS, E. A. Numerical limit analysis of three-dimensional slope stability problems in catchment areas. Acta Geotechnica, 11(6):1369-1383, 2016.

[24] CARRIÓN, M; VARGAS, E; VELLOSO, R ; FARFÁN, A. Slope stability analysis in $3 \mathrm{~d}$ using numerical limit analysis (nla) and elasto-plastic analysis (epa). Geomechanics and Geoengineering, p. 1-16, 042017.

[25] CHAHUA CRUZ, L. VARGAS, E; VAZ, L. Análise limite para o cálculo do fator de segurança de estabilidade de taludes utilizando programação cônica de segunda ordem. XXXIV Iberian Latin-American Congress on Computational Methods in Engineering, CILAMCE, Pirenópolis, GO,Brazil, 2013.

[26] FARFAN, A. Aplicação da análise limite a problemas geotécnicos modelados como meios contínuos convencionais e meios de coserat. Tese (Doutorado em Engenharia Civil)-Pontifícia Universidade Católica do Rio de Janeiro, Rio de Janeiro, p. 170, 2000.

[27] GARCIA ROJAS, J. VARGAS, E; VELLOSO, R. Análise limite numérica usando programação semidefinida e cônica de segunda ordem com aplicação em estabilidade de túneis rasos. Dissertação (Mestrado em Engenharia Civil)-Pontifícia Universidade Católica do Rio de Janeiro, Rio de Janeiro, 2018.

[28] TAPIA MORALES, M. Análise de confiabilidade de taludes em condições saturadas-não saturadas via análise limite no espaço cônico quadrático. Dissertação (Mestrado em Engenharia Civil)-Pontifícia Universidade Católica do Rio de Janeiro, Rio de Janeiro, 2014.

[29] NAVARRO, R. Comportamento plástico e os efeitos do envelhecimento sobre a tensão de escoamento. Revista Eletrônica de Materiais e Processos, 13:170-176, 2018.

[30] W, P. Recent developments of the mathematical theory of plasticity. Journal of Applied Physics, 20:235, 1949.

[32] MELAN, E. Zur plastizitat des raumlichen kontinuums. IngenieurArchiv, 9:116-125, 1938. 
[33] VON MISES, R. Mechanik der plastischen formaenderung von kristallen. Zeitschrift fär angewandte mathematik und mechanik, 29:445-458, 1928.

[34] NAYAK, G. C; ZIENKIEWICZ, O. C. Convenient form of stress invariants for plasticity. Journal of the Structural Division, 98(4):949-954, 1972.

[35] RIYAD, A. S. M; ROKONUZZAMAN, M ; SAKAI, T. Effect of using different approximation models to the exact mohr-coulomb material model in the fe simulation of anchor foundations in sand. International Journal of Geo-Engineering, 11(1):1-21, 2020.

[36] YU, H.-S. Plasticity and geotechnics, volumen 13. Springer Science \& Business Media, 2007.

[37] LYSMER, J. Limit analysis of plane problems in soil mechanics. Journal of the Soil Mechanics and Foundations Division, 96:1311-1334, 1970.

[38] SLOAN, S. Lower bound limit analysis using finite elements and linear programmming. International Journal for Numerical and Analytical Methods in Geomechanics, 12:67-77, 1988.

[39] BOTTERO, A; NEgRE, R; PASTOR, J ; TURGEMAN, S. Finite element method and limit analysis theory for soil mechanics problems. Computer Methods in Applied Mechanics and Engineering, 22:131-149, 1980.

[40] ABDI, R; DE BUHAN, P ; PASTOR, J. Calculation of the critical height of homogenized reinforced soil wall: a numerical aproach. International Journal for Numerical and Analytical Methods in Geomechanics, 18:485-505, 1994.

[41] MAKRODIMOPOULOS, A; MARTIN, C. Upper bound limit analysis using simplex strain elements and second-order cone programming. International Journal for Numerical and Analytical Methods in Geomechanics, 31:835-865, 2007.

[42] CASCIARO, R; CASCINI, L. A mixed formulation and mixed finite elements for limit analysis. International Journal for Numerical Methods in Engineering, p. 211-243, 1982.

[43] CHRISTIANSEN, E. Computation of limit loads. International Journal for Numerical Methods in Engineering, 17, 1981. 
[44] CHRISTIANSEN, E; ANDERSEN, K. Computation of colapse states with von mises type yield condition. International Journal for Numerical Methods in Engineering, 46:1185-1202, 1999.

[45] ZOUAIN, N; BORGES, L ; SILVEIRA, J. Quadratic velocity-linear stress interpolations in limit analysis. International Journal for Numerical Methods in Engineering, 98:469-491, 2014.

[46] ARAUJO, L. G. Estudo numérico de problemas de estabilidade em materiais geotécnicos através da análise limite. Tese (Doutorado em Engenharia Civil)-Pontifícia Universidade Católica do Rio de Janeiro, Rio de Janeiro, 1997.

[47] COUTO, C; FARFÁN, A. Estudo do fator de colapso em problemas geotécnicos. Dissertação (Mestrado em Engenharia Civil)-Universidade Estadual do Norte Fluminense Darcy Ribeiro, 2018.

[48] KRABBENHØFT, K; LYAMIN, A ; KRABBENHØFT, J. Mannual of Theory Optum G2. 2016.

[49] ZOUAIN, N; HERSKOVITS, J; BORGES, J ; FEIJÓO, R. A. An iterative algorithm for limit analysis with nonlinear yield functions. International Journal of Solids and Structures, 30:1397-1417, 1993.

[50] LYAMIN, A; SLOAN, S. Lower bound limit analysis using non-linear pro- gramming', international journal for numerical methods in engineering. International Journal for Numerical and Analytical Methods in Geomechanics, 55:573-611, 2002a.

[51] APS, M. Mosek optimization toolbox for matlab. 9.2.29, 2020.

[52] ANDERSEN, E; ROOS, C ; TERLAKY, T. On implementing a primaldual interior-point method for conic quadratic optimization. Mathematical Programming, 95(2):249-277, 2003.

[53] TIMOSHENKO, S; GOODIER, J. Theory of Elasticity, by S. Timoshenko and JN Goodier,... McGraw-Hill book Company, 1951.

[54] MARTIN, C; MAKRODIMOPOULOS, A. Finite-element limit analysis of mohr-coulomb materials in $\mathbf{3 d}$ using semidefinite programming. Journal of engineering mechanics, ASCE, 134(4):339-347, 2008.

[55] BRUNO, H. B; BARROS, G; MARTHA, L. F ; MENEZES, I. Formulação e solução de problemas de retorno à superfície de escoamento via 
programação cônica. Latin American Congress on Computational Methods in Engineering, CILAMCE, 2017.

[56] RAHAMAN, O; MOHAPATRA, D ; KUMAR, J. Bearing capacity of piles in nonhomogeneous clay using conic programming. Indian Geotechnical Conference, 2018.

[57] KRABBENHØFT, K; LYAMIN, A. Strength reduction finite-element limit analysis. Géotechnique Letters, 5(4):250-253, 2015.

[58] FERNÁNDEZ, F. Modelagem numérica de problemas geotécnicos de grandes deformações mediante o método do ponto material. Tese (Doutorado)-Pontifícia Universidade Católica do Rio de Janeiro, Rio de Janeiro, 2020.

[59] KUMAR, J; MOHAPATRA, D. Lower-bound finite elements limit analysis for hoek-brown materials using semidefinite programming. Journal of Engineering Mechanics, 143(9), 2017.

[60] RIBEIRO, W; ARAUJO, L ; NOGUEIRA, C. Aplicações da análise limite numérica a problemas de estabilidade axissimétricos em geotecnia. Dissertação (Mestrado em Engenharia Civil)-Universidade Federal de Ouro Preto, 2005. 